\title{
ANAT
}

2016 NIAC Phase I Study Journey to the Center of Icy Moons

\section{Enceladus Vent Explorer Concept}




\section{Study Team}

Jet Propulsion Laboratory, California Institute of Technology

PI: Dr. Masahiro (Hiro) Ono
Dr. Karl Mitchel
Dr. Aaron Parness
Kalind Carpenter
Dr. Aaron Curtis
Dr. Mitch Ingham
Dr. Charles Budney
Dr. Tara Estlin
Dr. Carolyn Parcheta
Dr. Renaud Detry
Jeremy Nash
Dr. Jean-Pierre de la Croix
Jessie Kawata

Dr. Kevin Hand

Università di Pisa

Saverio lacoponi

\section{Massachusetts Institute of Technology \\ Ellie Simonson}

\section{Acknowledgements}

This work was funded by the NASA Innovative Advanced Concepts (NIAC) program.

We thank Penny Boston, Peter Willis, Morgan Cable, Florian Kehl, Matt Heverly, Noah Warner, Steve Sell, and Sabrina Feldman for valuable inputs.

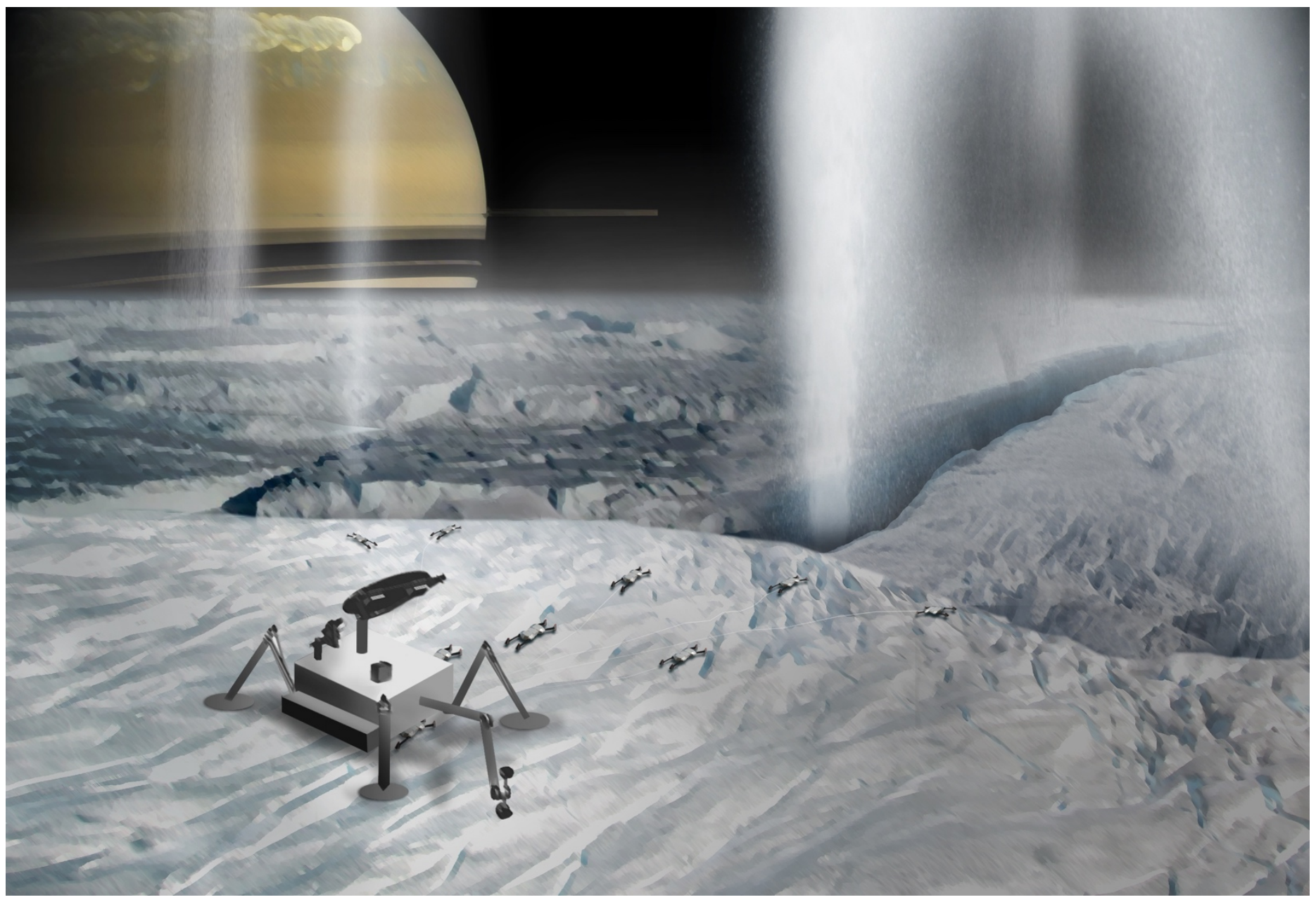




\section{Table of Contents}

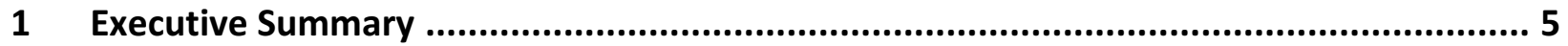

2 Mission Concept ……............................................................................................11

$2.1 \quad$ System Configuration ................................................................................................ 11

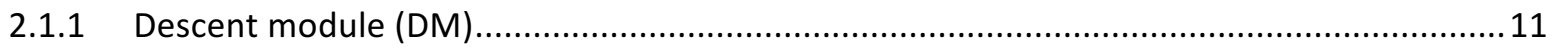

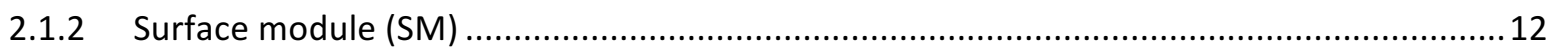

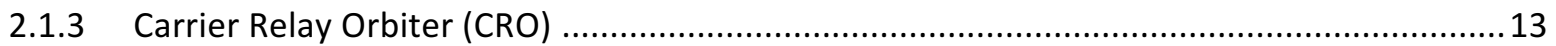

2.2 Mission Profile of EVE ...................................................................................................13

2.2.1 Launch, Cruise, Orbit Insertion, Landing .....................................................................13

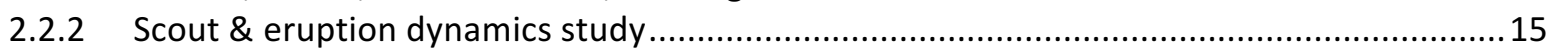

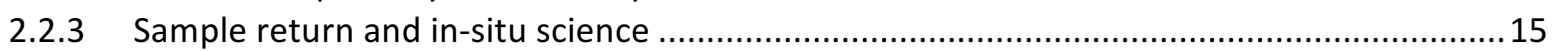

2.3 Comparison with existing concepts ...............................................................................16

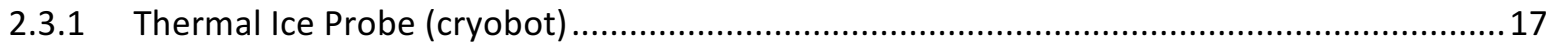

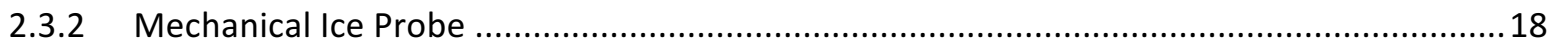

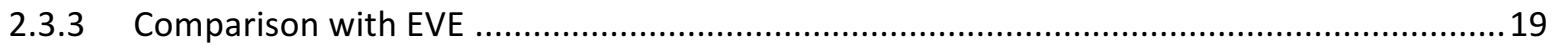

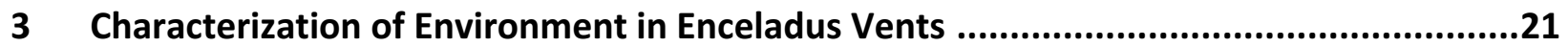

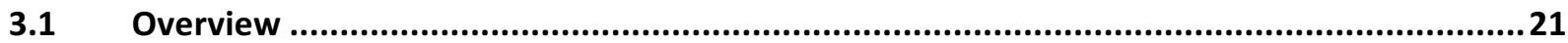

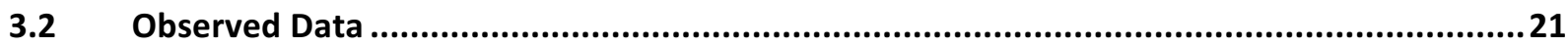

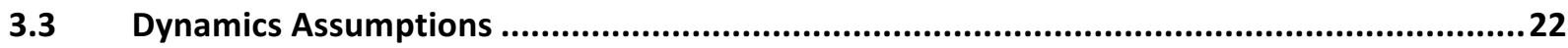

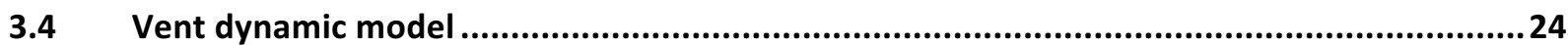

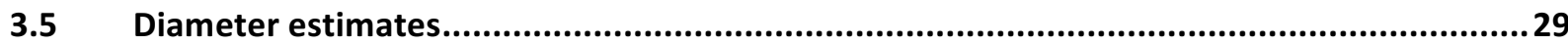

3.6 Nozzle shape estimations ................................................................................................ 30

3.7 Suggestions for future study to better constrain key parameters .......................................... 30

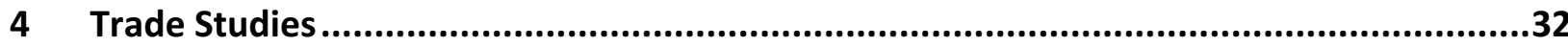

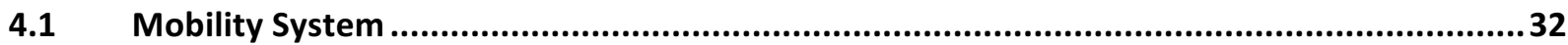

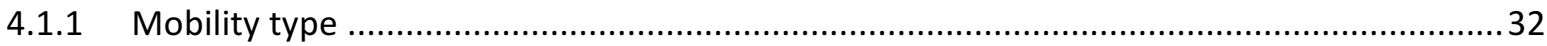

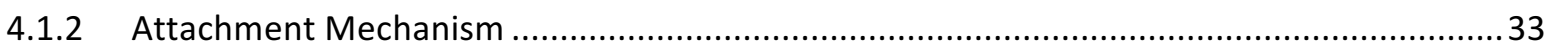

4.2 Power System ...............................................................................................................

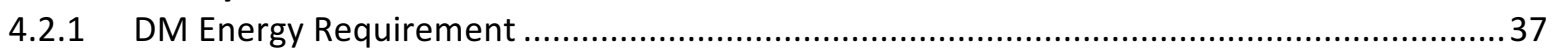

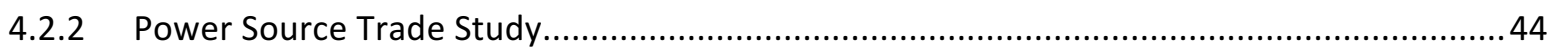

4.3 Tether System .............................................................................................................. 47

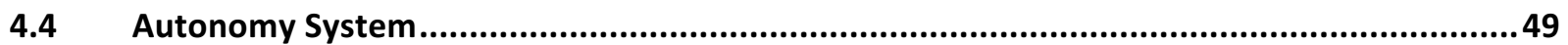

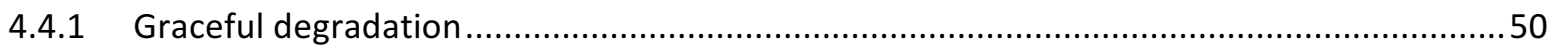

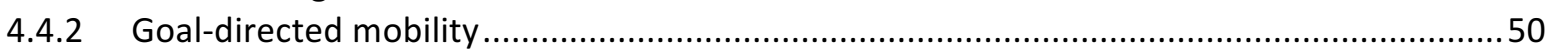

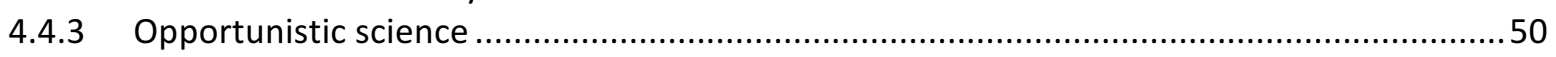

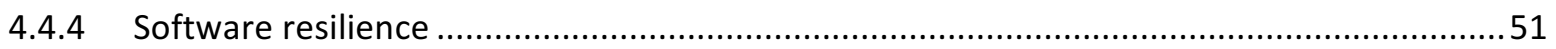

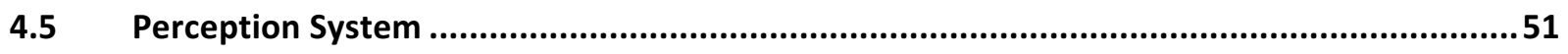

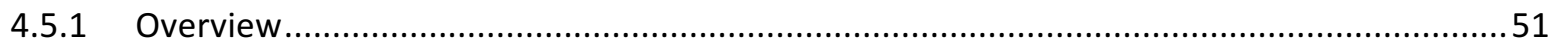

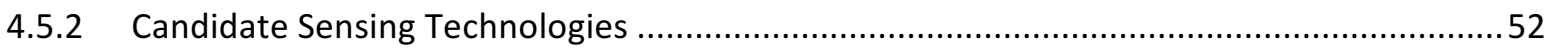

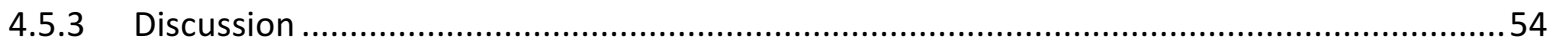

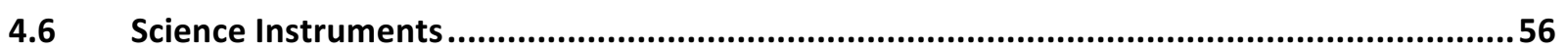

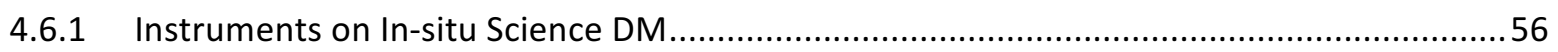

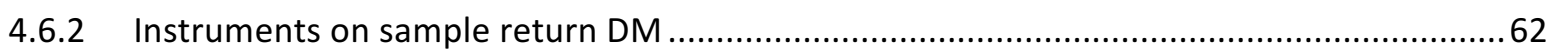

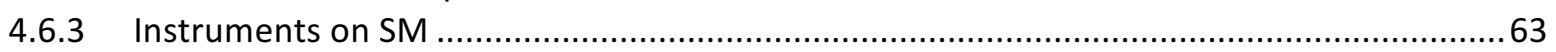

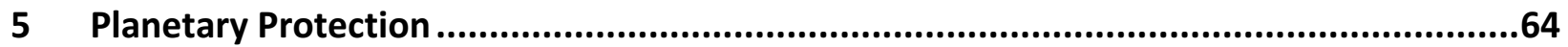

2016 NIAC Phase I Study: Journey to the Center of Icy Moons 3 


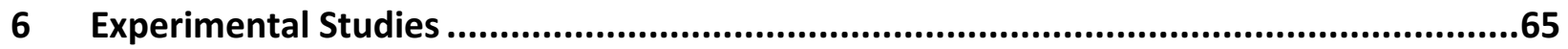

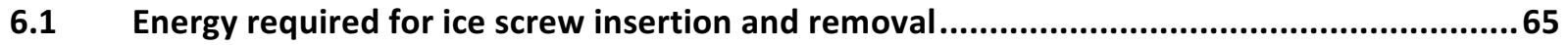

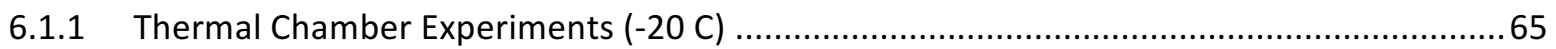

6.1.2 Conservative energy requirement estimate for the Insertion and Removal ......................68

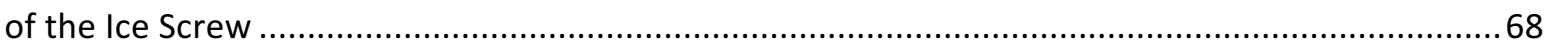

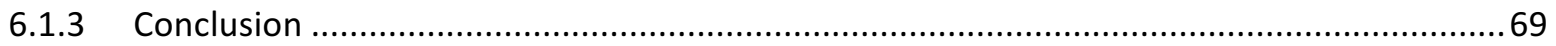

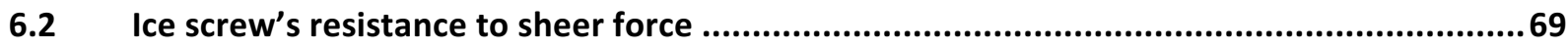

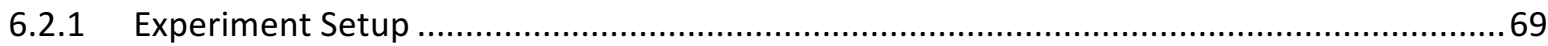

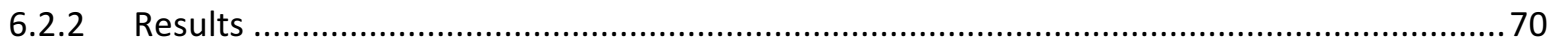

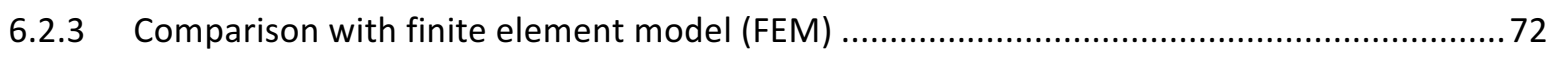

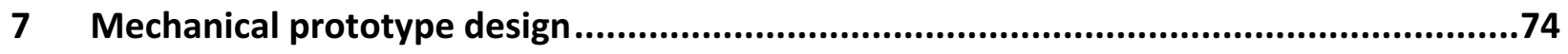

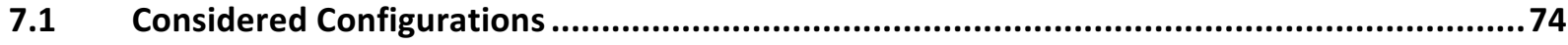

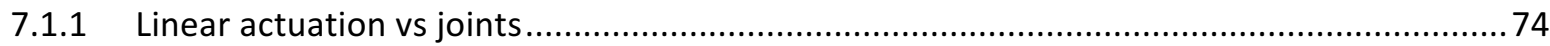

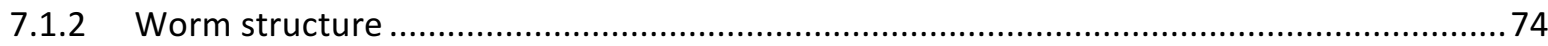

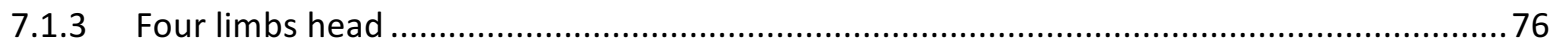

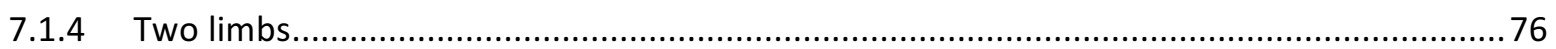

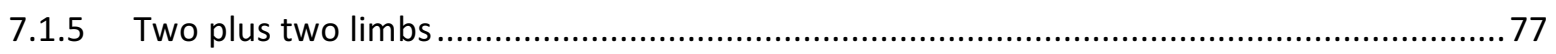

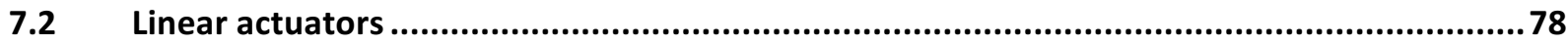

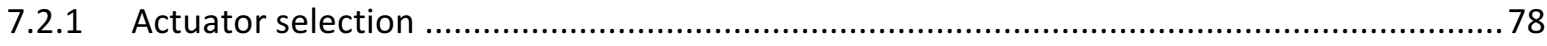

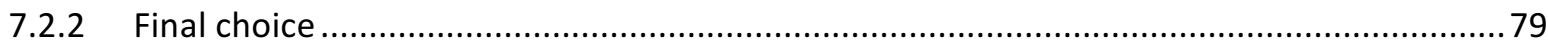

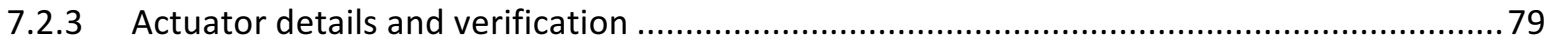

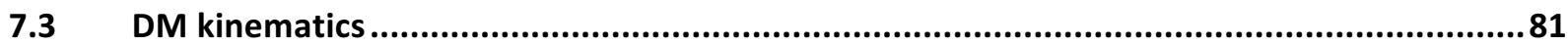

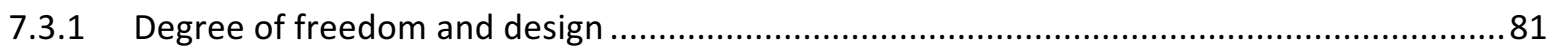

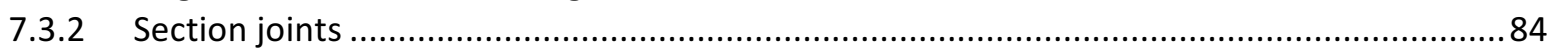

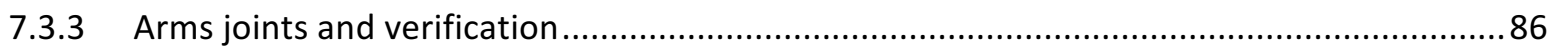

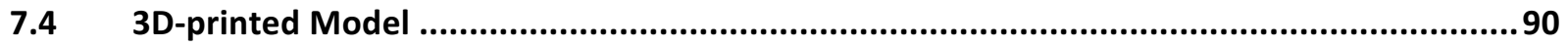

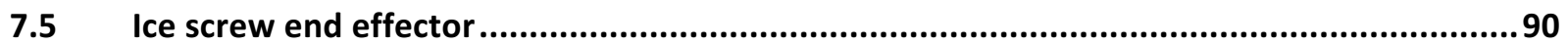

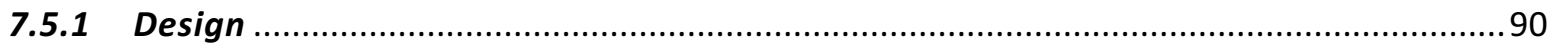

7.5.2 Assessment of screw insertion forces and torques ..................................................... 91

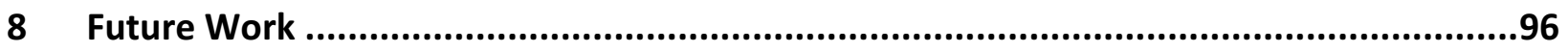

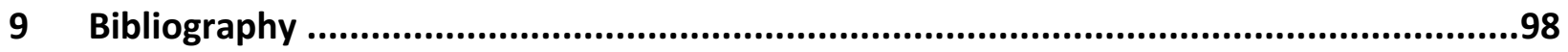




\section{Executive Summary}

Sometimes Nature acts as if it is waiting to be explored. For example, it secretly installed "doors" on the icy shell of Enceladus ${ }^{1}$ - vents through which water vapor and other materials are ejected from the subsurface ocean into outer space. The doors may serve as a natural pathway through the ice crust, which is tens of kilometers thick, to reach the extraterrestrial ocean. The doors may provide us with an opportunity to investigate a unique environment in the vent-conduit systems, which themselves could be habitable. The doors may lead us to the discovery of the second origin of life. The doors may lead us to the answer to mankind's ultimate question - "are we alone?" The doors are just 10 AUs away from us. Why would we leave them open and unexplored?

Our concept, Enceladus Vent Explorer (EVE), is a robotic pathfinder mission to enter these doors. EVE's goals are to descend into erupting conduits up to $\sim 2 \mathrm{~km}$ deep, characterize the unknown interior structure of the vent-conduit system, assess the accessibility to the subsurface ocean through the vent-conduit system, potentially reach the liquid interface, and perform astrobiology and volcanology observations in the vent-conduit system. EVE sends two types of modules: Surface Module (SM) and Descent Module (DM). SM is a lander that stays on the surface, while tens of small ( $\sim 3 \mathrm{~kg}, 10$ $\mathrm{cm}$ in width and $30 \mathrm{~cm}$ in length) DMs separate from SM, move to a vent, and descend into it. DMs rely on a power and communication link provided by SM through a cable. As the payload volume of DM is extremely limited, each DM can carry only a single miniaturized instrument. This limitation is complemented by heterogeneity. There are several types of DMs, all of which share the common mobility system but carry different instruments. For example, a "scout DM" creates a 3$D$ map of the geyser system with its stereo cameras and structured light. A "sample return DM" collects particles and ice cores in the vent and deliver them to the mass spectrometer in the SM. An "in-situ science DM" carries science instruments, such as a microscopic imager and a microfluidics chip for biosignature detection. DMs are sent either sequentially or in parallel.

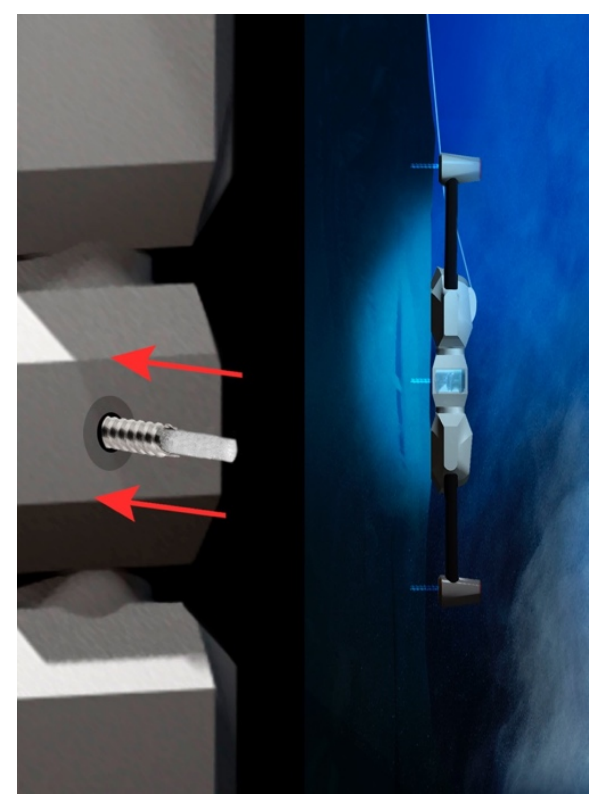

Figure 1 Sample return DM

The two greatest difficulties for EVE are the uncertainty in i) the dynamic pressure due to the upward flow and ii) the size of vent. Despite remote observations by the Cassini spacecraft, these

${ }^{1}$ As of the writing of this document, Enceladus is the only icy moon where vents have been directly observed by spacecraft. While recent Hubble observations of Europa have revealed a water vapor plume that could potentially have been vented from the subsurface ocean, its characteristics are poorly known and the existence of vents has not been confirmed. Hence, we set the primary target of our study to Enceladus. 
two parameters remain poorly characterized. Among the numerous unknowns associated with Enceladus vents, the one that has the greatest impact on the two key parameters is the eruption mechanism. Most hypothetical eruption models that have been proposed thus far fall into either of two categories, which we refer to as the relatively calm "boiling" models, and the more dynamic "cryovolcanic" models. Very roughly speaking, the "boiling" models assume that liquid water boils into vapor under the surface, while the "cryovolcanic" models assume that a fairly pure form of the ocean material ascends the conduit driven by exsolution and expansion of dissolved materials and phase changes. More detailed explanations of the two models will be provided shortly. Existing Cassini observations cannot resolve between the two models.
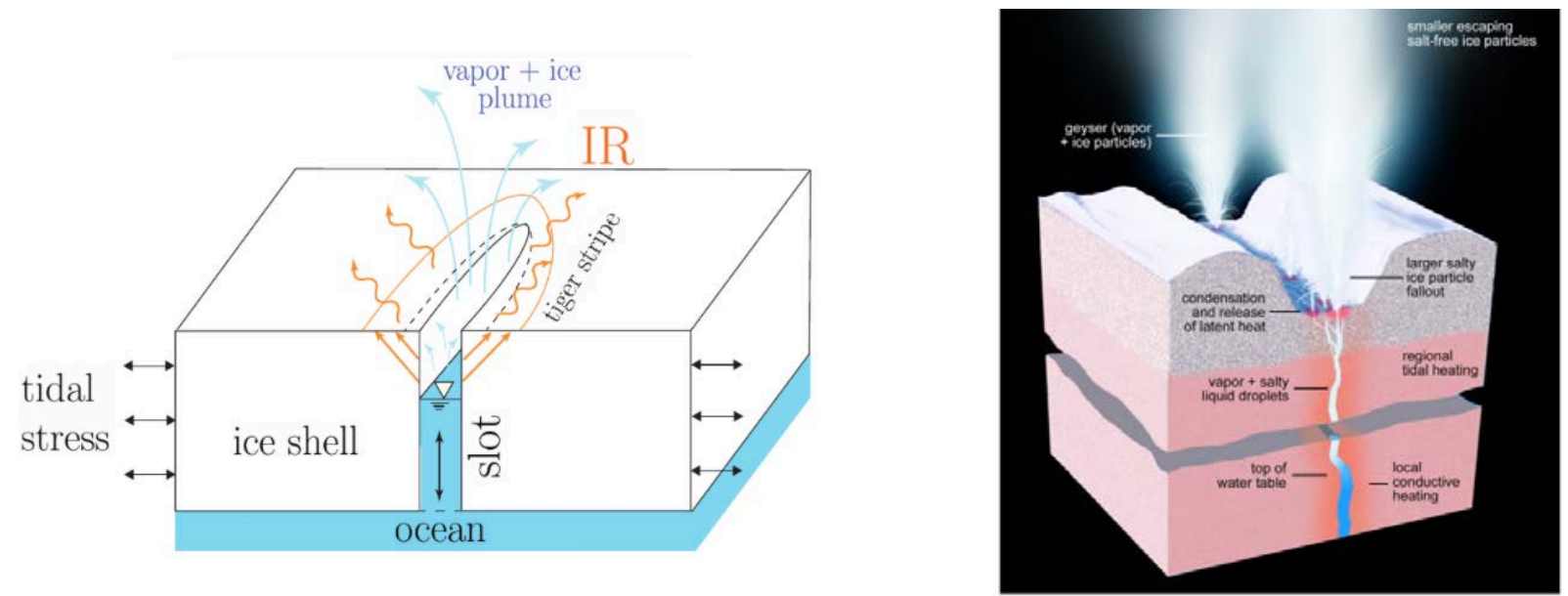

Figure 2 Left: A "Boiling" model by (Kite \& Rubin, 2016); right: a "cryovolcanic" model by (Porco, et al., 2014).

The "boiling" models are more favorable for EVE because they result in a greater vent size and lower dynamic pressure. The worst-case dynamic pressure is $\sim 10^{4} \mathrm{~Pa}$, which is well within the design range of DM. While the vent size is harder to constrain, the "boiling" models give orderof-magnitude greater estimates than the "cryovolcanic" model in general. For example, Kite and Rubin [2016] suggest "slots" of greater than 1-m in width. Therefore, EVE is likely feasible assuming the "boiling" models.

The "cryovolcanic" models are more problematic for EVE. Our preliminary analysis suggests that the dynamic pressure and the vent width could be anywhere between $10^{3}-10^{7} \mathrm{~Pa}$ and $1-30 \mathrm{~cm}$, respectively. The worst-case dynamic pressure is beyond the upper limit for DM. While some work suggests $10^{7} \mathrm{~Pa}$ dynamic pressure is likely unreasonable for geophysical reasons (D. Hemingway, pers. comm.) and more detailed analysis could better constrain the worst cases, we do not reject these possibilities. Therefore, the feasibility of EVE is undetermined with the current best knowledge under the "cryovolcanic" models.

Further study of Cassini data could better constrain parameter estimates of each model. In order to resolve between "boiling" and "cryovolcanic" models, additional orbital observations are likely needed. 
While observations from orbit around Enceladus could help provide some insight into the appropriate model, uncertainties in interior geometries and vertical profiles of dynamic pressure cannot be significantly reduced without descending into the vent. Therefore, the first mission into an Enceladus vent must face a chicken-and-egg problem: dynamic pressure and vent size remain uncertain until it descends into the vent, but designing a robot to descend into the vent requires knowledge of those parameters. Consequently, in order to maximize the chance of mission success, EVE's DM needs to be designed conservatively in terms of size and resistance to dynamic pressure.

Our trade study has determined that the DM configuration that is most robust to the uncertainties in vent parameters is a limbed robot with ice screws as the end effector. An anchoring mechanism is needed because the upward force exerted by the jet is orders of magnitude greater than the downward force exerted by Enceladus's weak gravity ( 0.01g). An ice screw is a hollow metal screw used by alpinists for ice climbing. As a byproduct, it produces an ice core when screwed in, which could be brought back by the sample return DMs. Since an ice screw can make a strong anchor with only one wall (as opposed to wedging, for example, which requires two walls), it is robust to uncertainty in the width of conduit. We validated by ice chamber experiments that ice screw end effectors can tolerate the force exerted by $\sim 10^{5} \mathrm{~Pa}$ dynamic pressure on the DM.

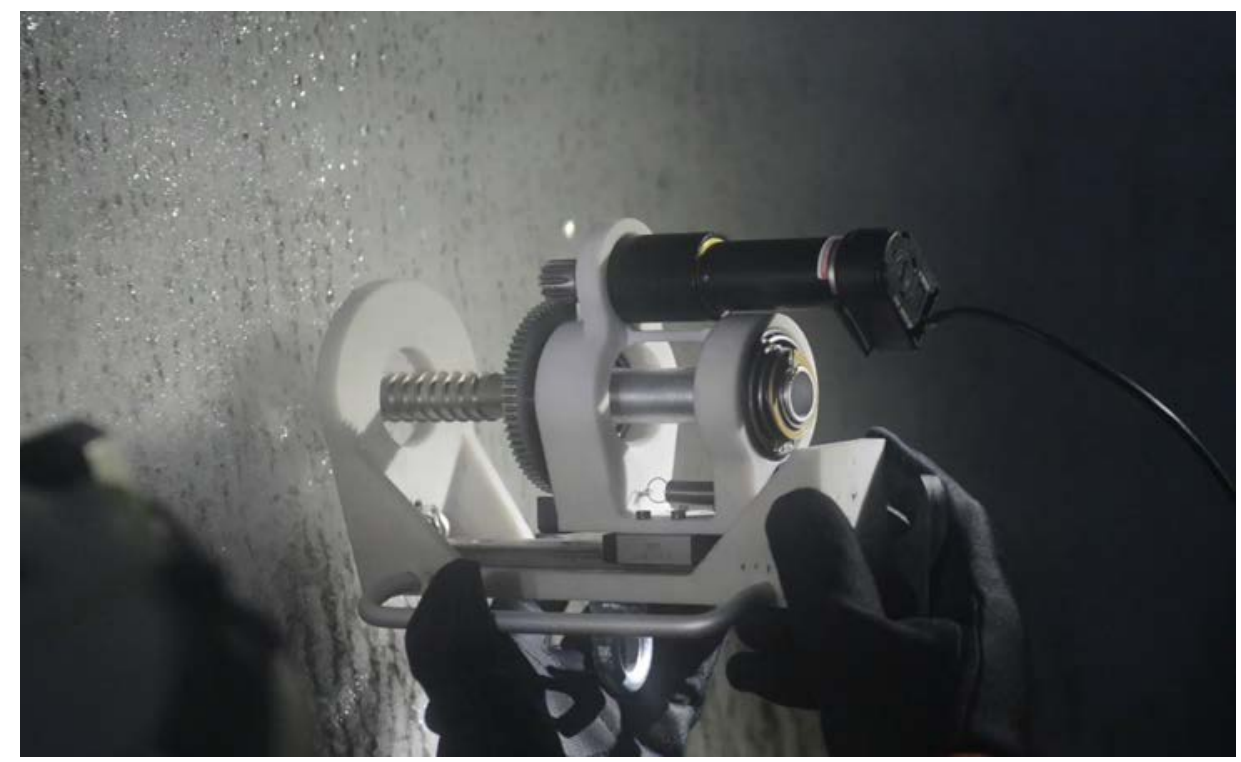

Figure 3. Test of a prototyped ice screw end effector in Helo Cave, a fumarolic ice cave on Erebus volcano in Antarctica.

Our trade study also concluded that the most realistic way to power DM is to provide power from SM through a cable because neither RTGs nor primary batteries that fit in the limited volume of DM can provide sufficient energy for the mobility system within a reasonable mission duration. A cable also eliminates difficulties in communication as well as in navigation for the return trip of sample-return DM. A three-section DM can accommodate up to $2 \mathrm{~km}$ of cable in its rear section. By adding a dedicated section for storing cable, it can accommodate up to $10 \mathrm{~km}$ of 
cable. The estimated speed of DM is $5.5 \mathrm{~m} / \mathrm{hr}$, given $10 \mathrm{~W}$ of power for mobility. If continuously operated, it can move $1 \mathrm{~km}$ in 7.5 days.

We use the proposed Europa Lander as a reference design for SM. The major difference from Europa Lander is the power source. Europa Lander uses a 45-kWh of primary battery, which weighs approximately twice of MMRTG, because the lifetime of the supporting Carrier Relay Orbiter (CRO) is limited to 30 days due to Europa's harsh radiation environment, thus a longerlived lander mission is difficult to justify (Hand, et al., 2017). In contrast, measurements indicate that the intensity of the trapped radiation environments of Saturn is much lower than the Earth's and is not likely to pose serious problems to spacecraft (Barth, et al., 2003). This justifies the use of RTGs to support a long mission.

Given this basic configuration, there is still a vast trade space to explore, particularly in the design of DM - size of the robot, DOF of each limb, choice of actuators, shape, etc. In particular, size is very important. The drag force by the flow is approximately proportional to the crosssectional area of the robot, but the mechanical force that the robot can apply in order to resist the drag also increases with the size. It is beyond the scope our Phase I study to optimize the design of DM. Instead, in order to obtain a reference point for the tolerable dynamic pressure, we developed a prototype design of DM with $\sim 10 \mathrm{~cm}$ in width. The prototype design only uses existing technologies/materials and commercially available mechanical parts (e.g., motors, gear boxes). It is a point design and not an optimal one, but it has been guided by experienced JPL mechanical engineers. The resulting design has four 5 DOF limbs, actuated by commercially available electrical motors. Each limb features a linear actuator, which is used to move DM's body against the flow. Each limb is designed to support up to $1000 \mathrm{~N}$ of force, meaning that DM can withstand up to $4000 \mathrm{~N}$ of

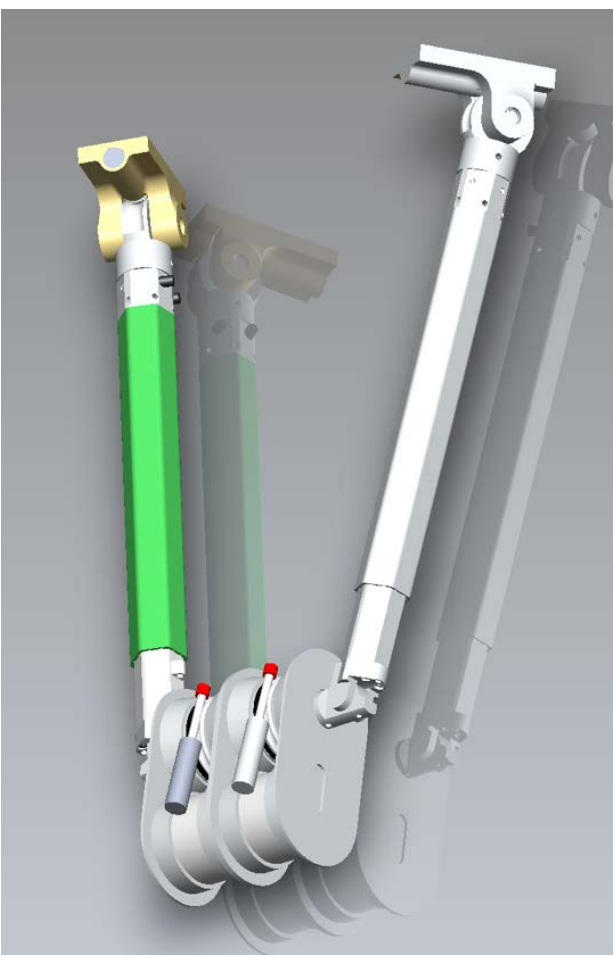

Figure 4 Prototype design of DM's front/rear section with two limbs force with its four limbs. The result of our computational fluid dynamics simulation suggests that the total force exerted on DM with $10^{6}$ Pa of dynamic pressure is $\sim 7400 \mathrm{~N}$. Therefore, the maximum tolerable dynamic pressure of this particular DM design is $5.4 \times 10^{5} \mathrm{~Pa}$. With safety margins, $\sim 10^{5} \mathrm{~Pa}$ would be the safe operational limit for this prototype design of DM. We note that the drag coefficient of the design is $\sim 1.5$, while that of typical automobiles, for example, is $0.2-0.3$. Clearly there is a significant room for design optimization, particularly in the aerodynamic shape. However, it is beyond the scope of this Phase I study to optimize the aerodynamic design of DM. 

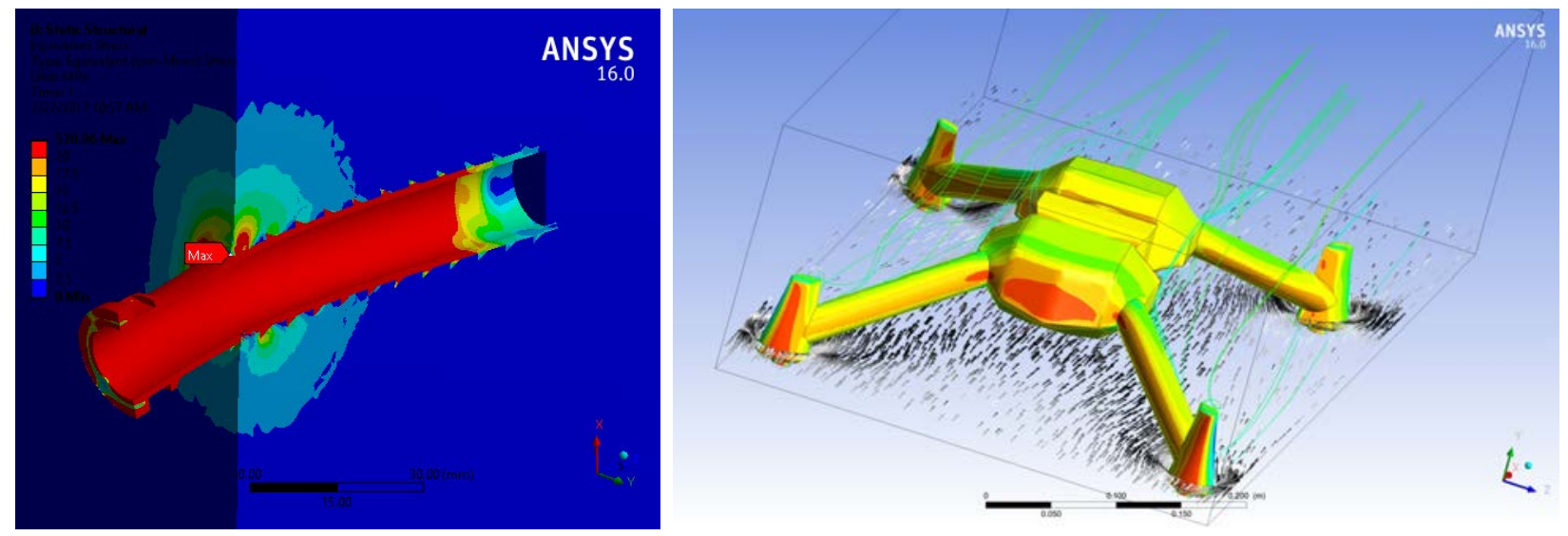

Figure 5 Left: FEM analysis of ice screw's sheer resistance; right: CFD analysis of aerodynamic drag.

Beyond the mechanical aspects of the EVE design, our study has considered the nature and degree of autonomy capabilities required to effectively execute the mission. Our study concludes that EVE must be a highly automated system in order to complete the mission within a reasonable duration. One of the most significant challenges for teleoperating DMs is the limited viewshed (geographical area that is visible from a location) in the vent. In Mars rover operations, the drive distance per operation cycle (Sol) of manual planning is limited by viewshed, which is typically $\sim 50 \mathrm{~m}$. In the vent, it would be tens of $\mathrm{cm}$. In case of Europa Lander, each operation cycle is $\sim 24 \mathrm{hr}$ long, which is constrained by the visibility of the CRO. Limiting DM motion to tens of $\mathrm{cm}$ per day, it would take decades to move one kilometer. In addition, operating tens of DMs manually would be a major challenge. In order to enable a realistic mission, required autonomy capabilities for DM include 3D mapping, localization, hazard detection and avoidance, route planning, activity planning, self-diagnosis, and recovery from failures. Agile science is highly desired to not miss interesting science opportunities during the traverse. Cooperative autonomy with multiple DMs is also important. The limited computational capabilities onboard the DM are not a limiting factor, since DM can access computational resources on SM through the cable. Furthermore, DMs can communicate with each other via SM. Hence, SM serves as the "information hub" during the automated operation.

In summary, the feasibility of EVE depends on the eruption mechanism, which at this point is unknown. Assuming the "boiling" model, in which the expected dynamic pressure is $<10^{4} \mathrm{~Pa}$ and the width of the vent is likely to be wider than $10 \mathrm{~cm}$, our prototype DM is feasible, hence the EVE concept is likely feasible. However, given the "cryovolcanic" model, in which the dynamic pressure and vent width could be anywhere between $10^{3}-10^{7} \mathrm{~Pa}$ and $1-30 \mathrm{~cm}$, respectively, the feasibility of EVE is undetermined.

Further study of the cryovolcanic model based on Cassini's observations, as well as optimization of DM design, would allow us to determine the feasibility of EVE, or at least to have a better understanding of the risks associated with the concept. In the near-term, there is still much we can do with existing data. For example, characterization of the upper limits of ocean pressure and volatile fractions could put additional constraints on dynamic pressure in the vent. Multi-species thermochemical/exsolution models would allow detailed tests of current 
conceptual models for ascent and eruption, and may allow for enhanced interpretation of plume mass spectrometric results. Further exploration of the design space of DM, in particular the optimization of aerodynamic shape and actuation mechanisms, could allow greater resistance to dynamic pressure.

Among our set of mid-term goals, resolving between the "boiling" and "cryovolcanic" models is the highest priority. This could be done by a future orbital mission such as ELF (Enceladus Life Finder). Very high resolution imaging combined with extremely sensitive passive emission spectrometry at sub-mm/THz wavelengths at optimized phase angles may give the highest potential for resolving the models. High resolution imaging of individual vents will also help identify the landing target for EVE.

Finally, we would like the readers to recall the excitement of Jules Verne's Journey to the Center of the Earth. In the classic science fiction novel, Professor Otto Lidenbrock and his company descend into an Icelandic volcano, discover a vast subsurface ocean, and encounter exotic life. In the near future, EVE could make such a fictional adventure real, not on Earth but in the frosty and mysterious world of Enceladus. 


\section{Mission Concept}

\subsection{System Configuration}

EVE consists of three modules: Descent Module (DM), Surface Module (SM), and Carrier Relay Orbiter (CRO). This Phase I study mostly focused on the DM as it is the most unique feature of EVE. For SM and CRO, we use Europa Lander and its CRO as reference designs, with minor modifications.

\subsubsection{Descent module (DM)}

A DM is a small $(<3 \mathrm{~kg}$ ) robot, whose role is to descend into the vent. As a result of our trade study describe in Section4, the most promising DM configuration is a limbed robot with ice screws as the end effector. As shown in Figure 6, a DM's standard configuration consists of three sections. The nearly identical front and rear sections accommodate mechanical components to actuate two limbs. A pair of cameras and structured light projector is mounted on the front section for perception (see Section 4.5 for details), and a tether spool is accommodated in the rear section. The middle section accommodates instruments.

An EVE mission involves tens of heterogeneous DMs, all of which have identical front and rear sections but carry different instruments in its middle section. A "scout DM" carries extra mapping devices and creates a detailed 3-D map of the geyser system with its stereo cameras and structured light. A "sample return DM" has an additional ice screw in its middle section, which is used to create and store an ice core. Alternatively, it can have a device to collect particles in the vent. In either case, a sample return DM returns to SM for analysis. An "in-situ science DM" carries miniaturized science instruments in its middle section, such as a microscopic imager and a microfluidics chip for bio-signature detection (See Section 4.6 for the discussion on science instruments).
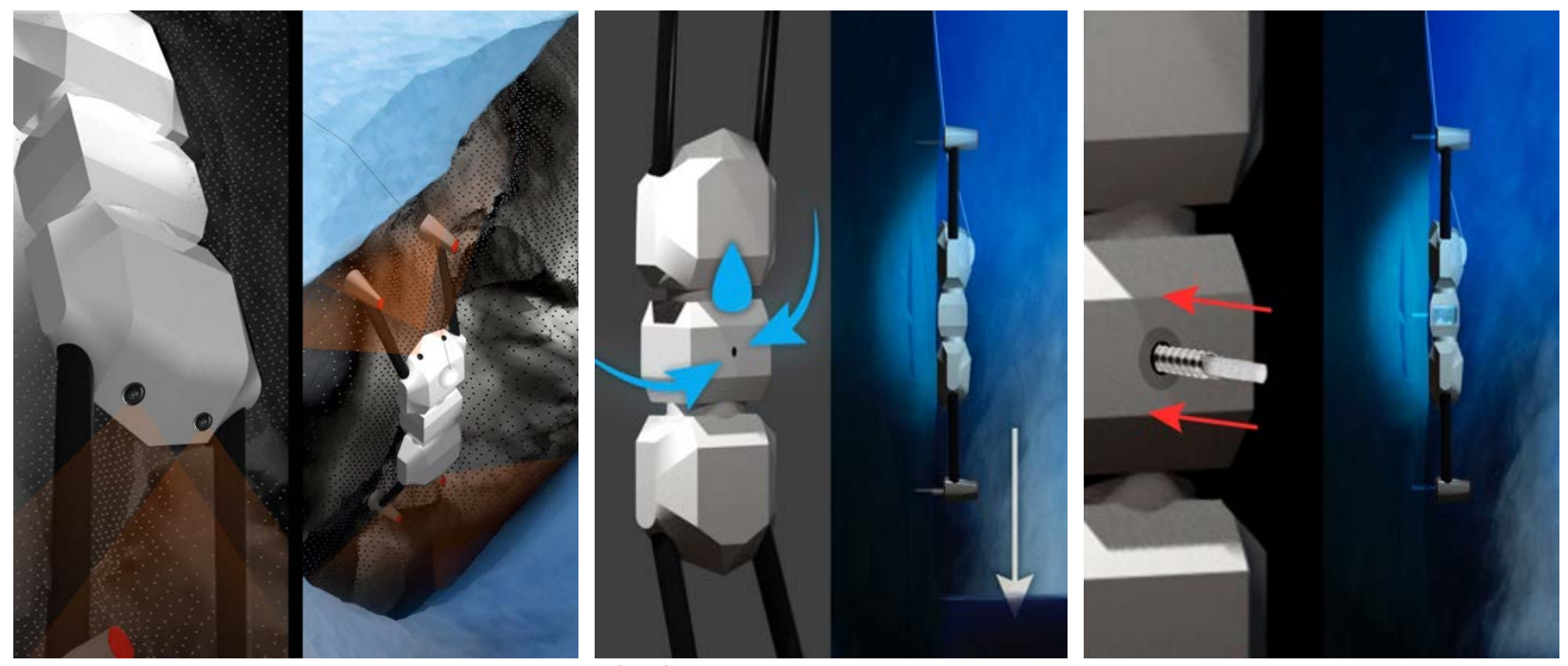

Figure 6 Scout DM (left), in-situ science DM, and sample return DM 
A DM could potentially have a train of multiple middle sections (with potential addition of limb sections) - essentially making it a "snake bot." This idea is attractive because adding sections would not significantly reduce the ability to traverse through vents or conduits, nor increase aerodynamic drag. However, due to the limitation of time and resources in the Phase I study, we only performed analysis of the three-section configuration.

One idea is to make the middle section interchangeable, so that a DM can transform to a different type. This will give significant flexibility operation. For example, an in-situ science DM can choose the best instrument based on the observations by scout DMs. A scout DM can transform into a sample return or an in-situ science DM on its second trip. However, it is beyond the scope of Phase I study to explore the mechanical implementation of an interchangeable middle section.

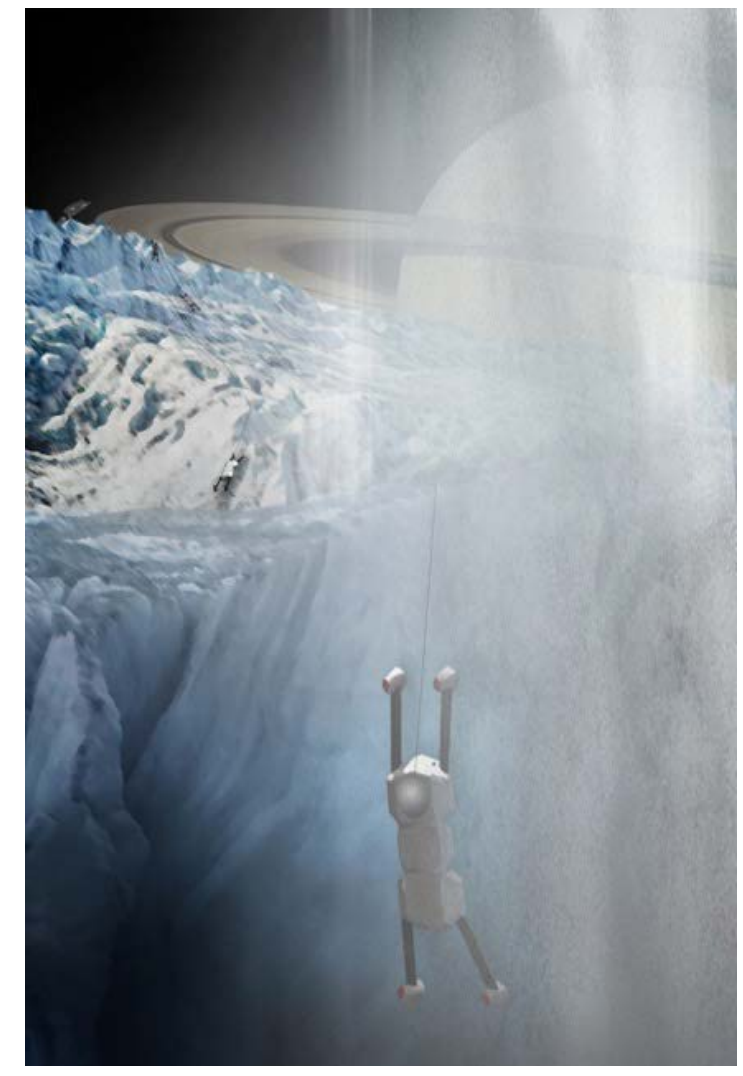

Figure 7 Descent of DM

\subsubsection{Surface module (SM)}

The SM's role is to deliver DMs to the surface of Enceladus and provide DMs with power and communication link through tethers. We use the Europa Lander (Hand, et al., 2017) as a design reference. The Europa Lander is powered by a $45-\mathrm{kWh}$ primary battery with $20+$ days of surface operation time. The decision to use this battery technology was based on the lifetime of the CRO being limited $\sim 30$ days due to Europa's harsh radiation environment, thus a longer-lived lander mission is difficult to justify. This is not the case for Enceladus. Measurements indicate that the intensity of the trapped radiation environments of Saturn is much lower than the Earth's and is not likely to pose serious problems to spacecraft (Barth, et al., 2003). This justifies the use of RTG to support a long mission ( $>1 \mathrm{yr}$ ). In the proposed design of Europa Lander, the mass of $45 \mathrm{kWh}$ of primary battery is $\sim 95 \mathrm{~kg}$ including packaging ${ }^{2}$, which is roughly equivalent to two MMRTGs (45 kg each). Each MMRTG provides $125 \mathrm{~W}$ at the start of mission and $100 \mathrm{~W}$ after 14 years.

The Europa Lander would carry $42.5 \mathrm{~kg}$ of science instruments as well as a Phoenix-derived sample collection system with a mass of $\sim 26 \mathrm{~kg}^{3}$. With this payload mass, the SM of the EVE can carry up to 20 DMs (assuming $3 \mathrm{~kg}$ each). Alternatively, it can carry a lesser number of DMs and science instruments, such as mass spectrometer, which is used to analyze samples brought back by the sample return DMs.

\footnotetext{
${ }^{2}$ Personal communication with Steve Sell, JPL

${ }^{3}$ Personal communication with Noah Warner, JPL 
A solar-powered SM is possible, but the landing site is constrained by the condition of sunlight. Given the rich topology of the Tiger Stripes in which the vents are located, finding a suitable landing ellipse would be a major difficulty. A more detailed trade study of power options is discussed in Section 4.2.

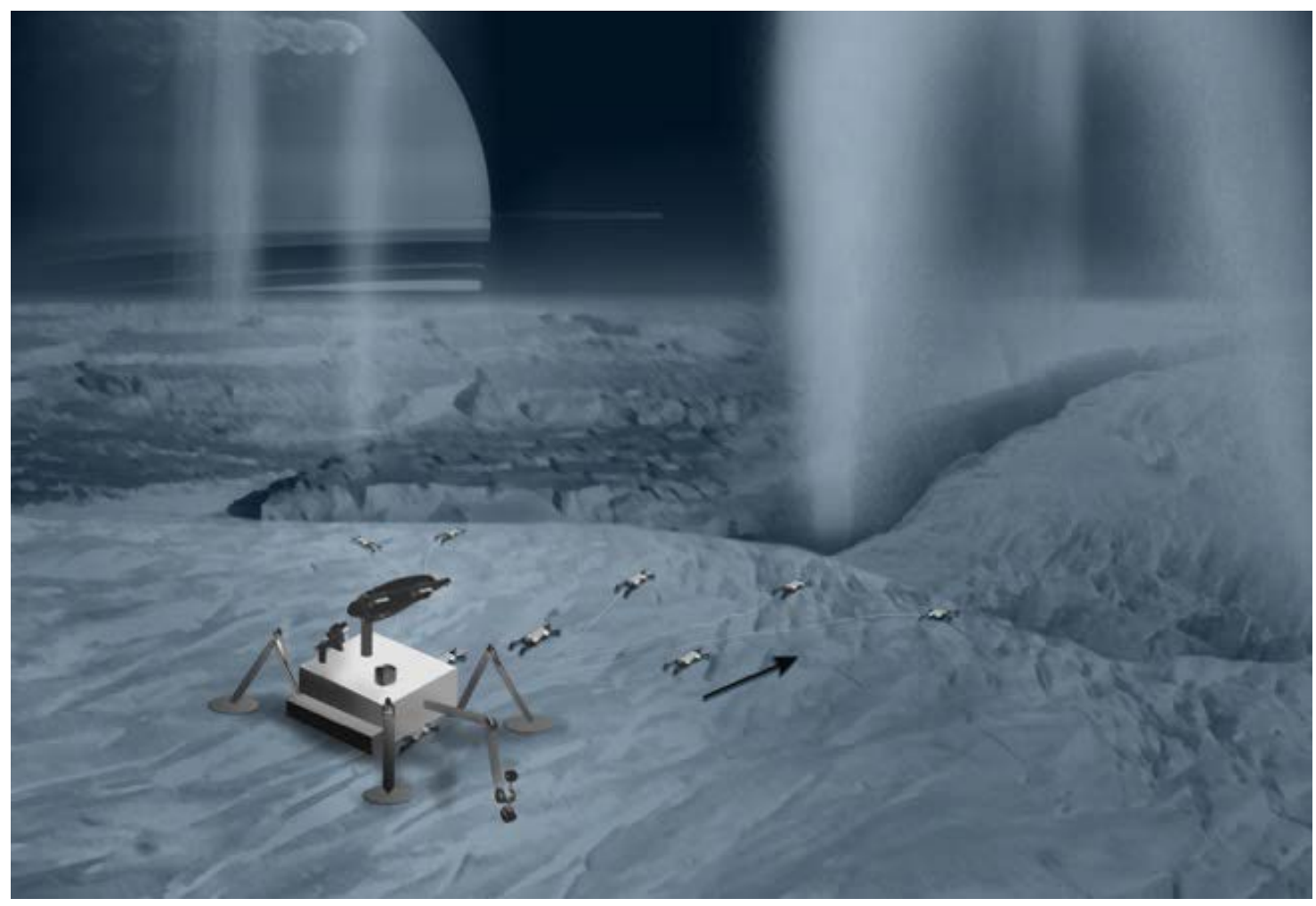

Figure 8 SM deploying DMs

\subsubsection{Carrier Relay Orbiter (CRO)}

Like the CRO of the Europa Lander, the role of EVE's CRO is to deliver SM-DMs to Enceladus orbit, and provide communication relay after landing. Due to the weak gravity of Enceladus, EVE's CRO would co-orbit with Enceladus (instead of orbiting Enceladus) at a slightly different inclination so that it can cover the south polar region over a half of Enceladus's orbital period (1.37 days). Like the proposed Enceladus Life Finder's mission, EVE's CRO can be solar powered.

\subsection{Mission Profile of EVE}

\subsubsection{Launch, Cruise, Orbit Insertion, Landing}

EVE's mission profile would be similar to that of Europa Lander up to landing (Figure 9). After being launched by an SLS-class launch vehicle, it's voyage to the Saturnian system would involve deep space maneuvers, Earth/Venus flybys, and a Jupiter flyby. Initially inserted to a highly elliptic orbit around Saturn, it incrementally lowers the aposaturnium point through Titan flybys, and finally co-orbits with Enceladus. Since the Saturnian system has only one moon that is massive enough for a changing orbit (Titan) as opposed to four in the Jovian system, the orbital transition 
may take a longer time than Europa Lander. In addition, the significantly weaker gravity of Enceladus would require a greater $\Delta \mathrm{V}$ for the orbit insertion to Enceladus's orbit around Saturn. A detailed orbital analysis is a future work.

Europa Lander's estimated landing accuracy is $100 \mathrm{~m}$ (Hand, et al., 2017). The significantly improved landing accuracy compared to Mars landing is primarily due to the lack of atmosphere, and the same condition applies to Enceladus. Hence, we assume $100 \mathrm{~m}$ landing accuracy for EVE.
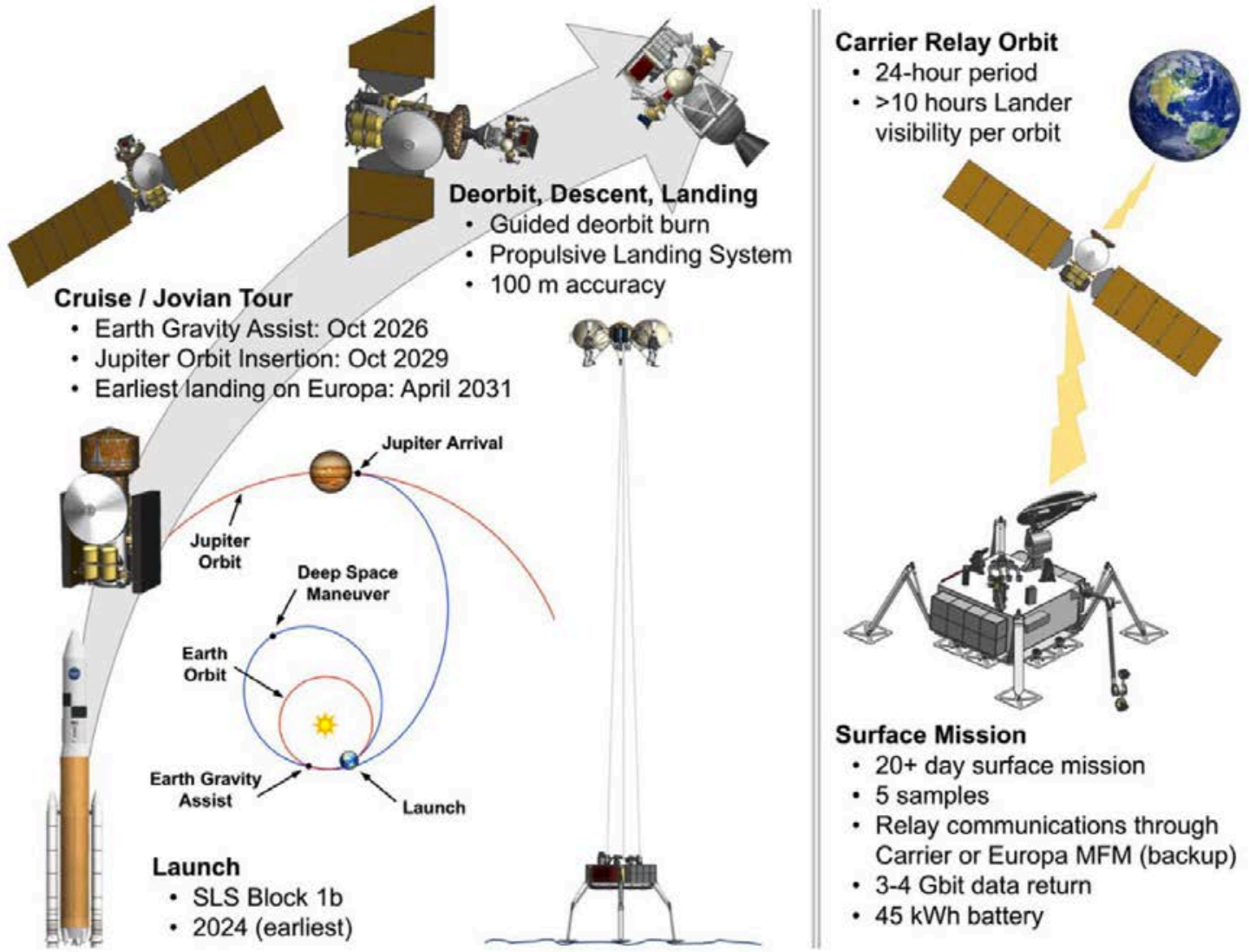

Figure 9 Key features of Europa Lander mission, from launch to landing. The EVE mission would have a similar mission profile up to landing. Image taken from (Hand, et al., 2017).

The landing site will be selected based on orbital observations. The selection is based on:

1) Landing safety (smooth flat terrain over $100 \mathrm{~m}$ radius),

2) The number of vents reachable from the landing point ${ }^{4}$, and

\footnotetext{
${ }^{4}$ Having multiple vents reachable from the landing point is desirable because it increases the chance that at least one of them is feasible for descent. However, based on current observations, multiple accessible vents within a few hundred meters unlikely. If there are discrete vents, as proposed by (Porco, et al., 2014), there are of order 100 of them spaced over $500 \mathrm{~km}$ of vent system. This would make a mean distance of order a few $\mathrm{km}$. Furthermore, if there were multiple vents within a few hundred meters, the probability is that they are very small ones carrying little flux, most likely of order $100 \mathrm{~g} / \mathrm{sec}$ or less.
} 
3) Estimated size (indicated by mass flux) and dynamic pressure of the vents.

Once landed, SM takes 360 panorama images to localize itself and identify accessible vents from the landing points. The panorama image is also used by ground operators to plan a path to the vents for DM.

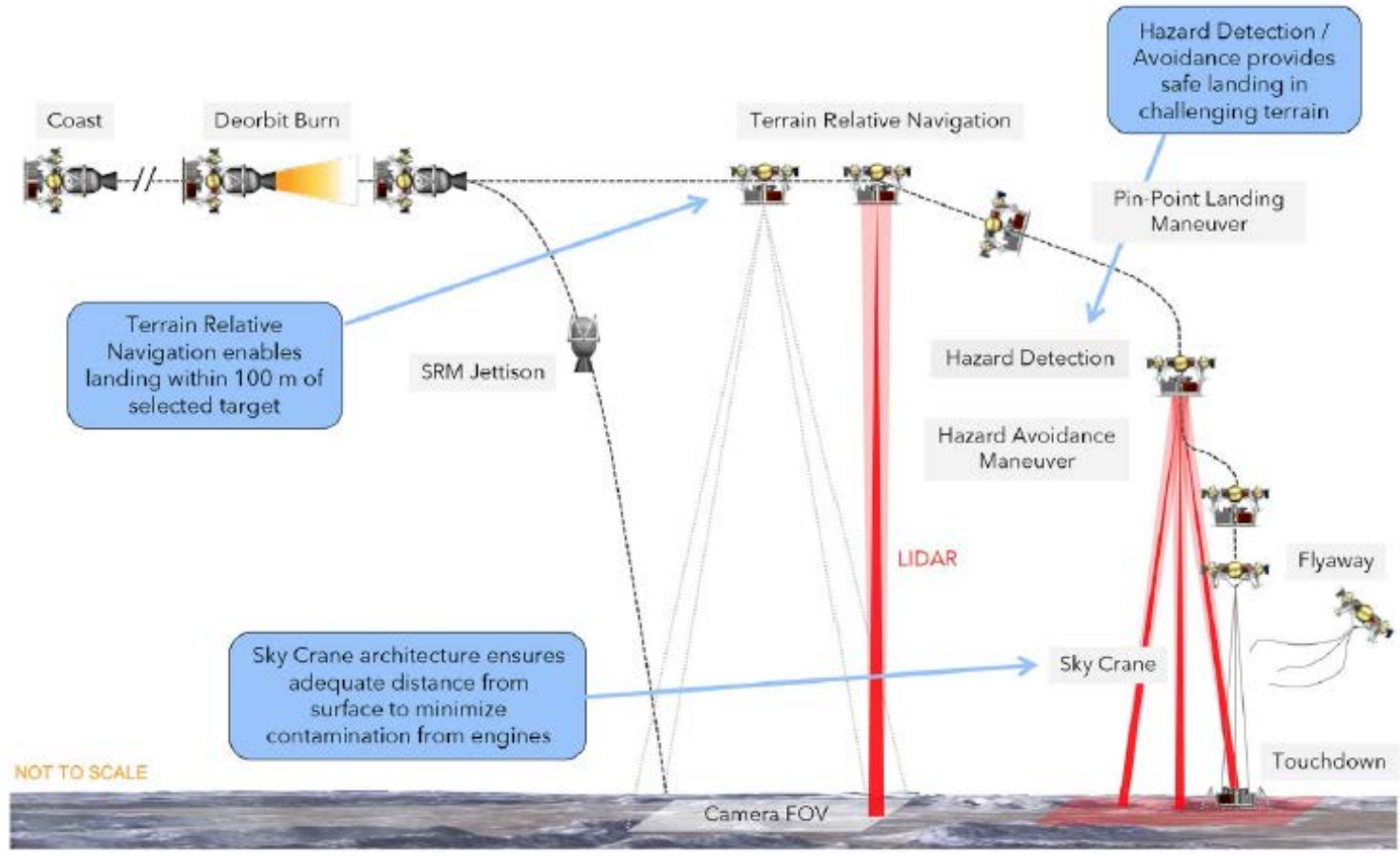

Figure 10 Europa Lander's deorbit, descent, and landing sequence. EVE will have a similar landing sequence.

\subsubsection{Scout \& eruption dynamics study}

First, SM deploys a few scout DMs. Each DM travels to the mouth of different vents (if multiple vents are within the reach) to assess the accessibility based on the visual observation of the size of the vent, the observed mass flux, and the observed flow speed. Once it finds a promising one, it goes into the vent while making a 3D map of the interior of the vent. If it finds the path is blocked either by obstacles or a decreased width of vent, it backs up and tries another vent. If it successfully goes past the chocking point, which is the most critical point for mobility, this vent is selected for further study. Some scout DM stays in the vent and continue the mapping for volcanology study as well as to create a map for following DMs. If DM's middle section is interchangeable, some scout DMs return to SM and get converted to sample return or in-situ science DMs.

\subsubsection{Sample return and in-situ science}

SM deploys sample return and in-situ science DMs. They follow the optimal path identified by the scout DMs. The sample return DMs collect an ice core, dust, and potentially liquid samples in 
the vent/conduit, and return to SM. The samples are analyzed by the mass spectrometer on SM to look for signs of life. Sample return DM has a binary nature: if it can successfully return to SM, there will be a significant science gain. Otherwise, the science gain is zero. Having multiple DMs helps hedging the risk. DMs pick sample from a diverse range of depth. Some pick samples from a shallow depth in order to secure minimum science return. Some try to return samples from a greater depth - a high-risk, high-return trip. Each DM can make multiple trips. Once a baseline goal is achieved, DMs can aim for more challenging destinations or sample from different vents, if there are multiple accessible vents.

The in-situ science DMs perform scientific observations along the way, and go as deep as possible to meet the liquid interface. Interchangeable payload gives flexibility in in-situ science. For example, if scout DMs find that the liquid interface is within an easily reachable depth, we can put microfluidics package on in-situ DMs to detect life in liquid. The observations by an in-situ DM can inform following DMs to choose the best instruments.

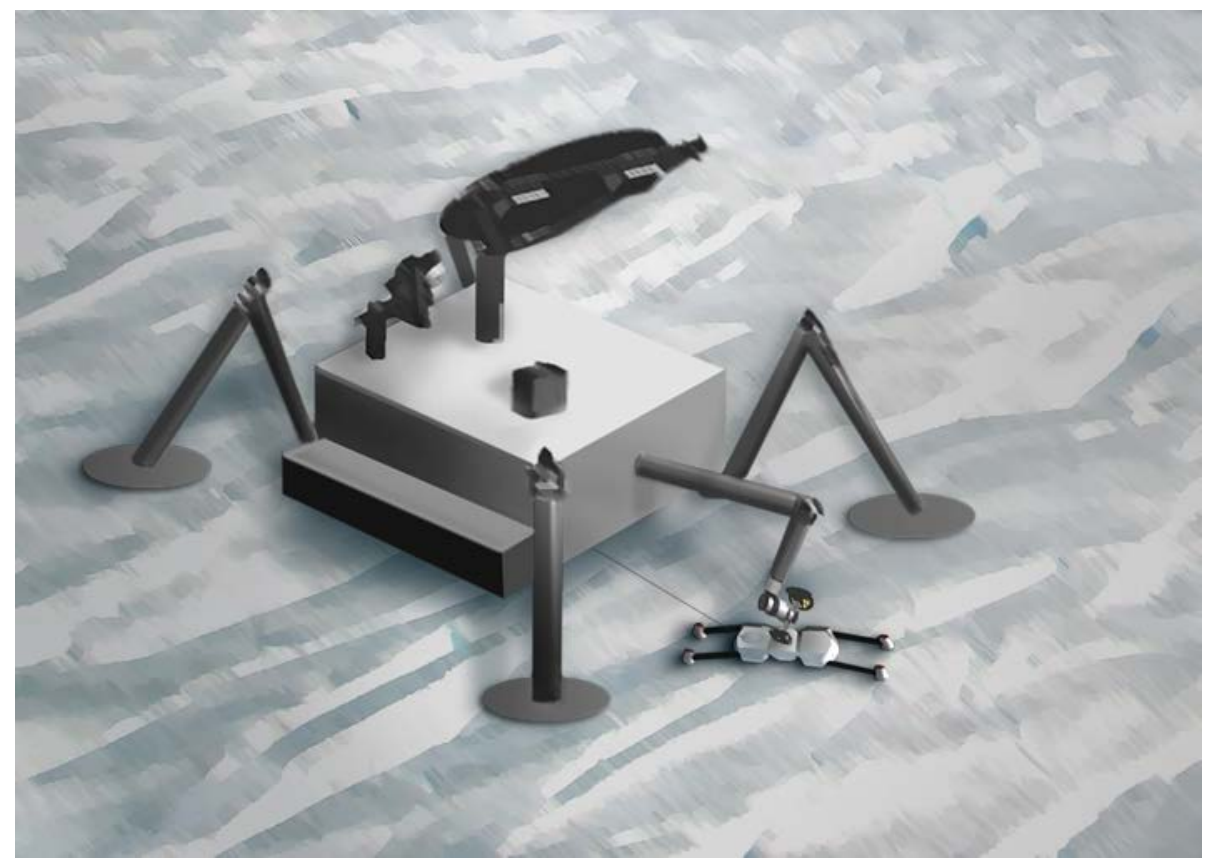

Figure 11 Sample retrieval from a sample return DM

\subsection{Comparison with existing concepts}

The goal of EVE is not to access the subsurface ocean, but to assess the accessibility of the ocean by understanding the vastly unknown conditions in the vent and conduit. However, it would be useful to compare existing concepts that aim to access the subsurface ocean because it helps understanding the advantage and disadvantage of using the vent-conduit system as a pathway to the ocean. 


\subsubsection{Thermal Ice Probe (cryobot)}

Cryobot (Zimmerman, et al., 2001) is a technology concept to melt through the ice shell. It is a torpedo-shaped vehicle as shown in Figure 12. While various designs have been proposed, (Zimmerman, et al., 2001) provided a detailed study and design of Cryobot for Europa and the Mars polar cap. Their design is $1.25 \mathrm{~m}$ in length and $12 \mathrm{~cm}$ in diameter, which melts ice using both passive heaters and a water jet heated by $1 \mathrm{~kW}$ of heat produced by RTG and descends by its own weight. Communication is provided by a surface module through a tether, or alternatively, a series of releasable communication pucks. The greatest advantage of Cryobot is its simplicity. However, melting ice is very energy inefficient, because heat quickly dissipates through ice. (Zimmerman, et al., 2001) estimate that, with a $1 \mathrm{~kW}$ heat source, of which 0.6-0.8 kW can be used for melting, the rate of progress is $1 \mathrm{~m} / \mathrm{hr}$, or 14 months for $10 \mathrm{~km}$ if operated continuously (Zimmerman, et al., 2001). The energy efficiency is $\sim 7.0-2.8 \mathrm{MJ}$ per meter of descent ${ }^{5}$. Sediment accumulating in front of the vehicle is also a challenge, but mitigated with water jets. In addition, while this concept works under Europa's gravity $(0.13 \mathrm{~g})$, it is not clear if it works under Enceladus's weaker gravity $(0.01 \mathrm{~g})$.

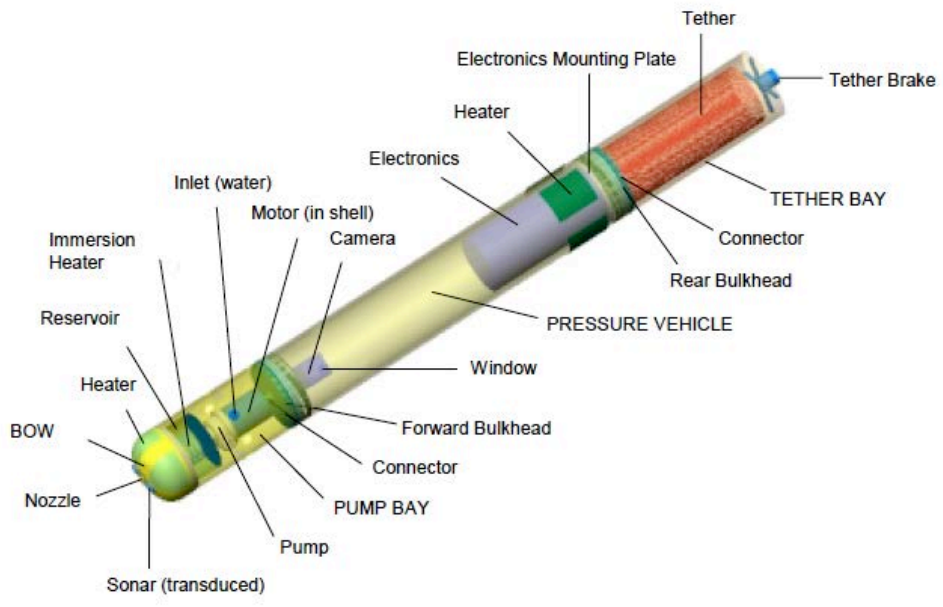

Figure 12 Cryobot proposed by (Zimmerman, et al., 2001)

\footnotetext{
${ }^{5}$ The speed estimate by (Zimmerman, et al., 2001) appears to be wrong because the $2.8 \mathrm{MJ} / \mathrm{m}$ energy requirement is less than the energy needed for melting ice with a $12 \mathrm{~cm}$ diameter probe $(3.8 \mathrm{MJ} / \mathrm{m})$. Furthermore, this does not take heat dissapation into account. For these reasons, we suspect that, in practice, the speed of the melt probe would be an order-of-magnitude slower than their $1 \mathrm{~m} / \mathrm{hr}$ estimate.
} 


\subsubsection{Mechanical Ice Probe}

The ice probe concept proposed by (Wilcox, et al., 2017) features a circular saw blade that protrudes through a slot in a hemispherical turret dome at the bottom of the probe, such that the blade cuts the ice as it spins. The ice chips would be thrown up through the slit into the turret and would be melted by the heat provided by General Purpose Heat Source (GPHS) modules containing Pu-238. The meltwater drains into sumps on either side of the sawblade, from which the meltwater would be pumped out to the rear of the probe. The main body of the probe contains a spool of aluminum tubing that would be dispensed from within the probe to the surface module lander. This tubing is nominally $1-3 \mathrm{~mm}$ in outside dimension with integral insulated electrical wires around the center hole. This tube would pneumatically transport small (mm-scale) single-use canisters containing meltwater samples from the probe to the surface for analysis.

Mechanically cutting ice is significantly more energy efficient than melting it. Required energy is $21.1 \mathrm{MJ} / \mathrm{m}^{3}$ for mechanical cutting and $333 \mathrm{MJ} / \mathrm{m}^{3}$ for melting without heat dissipation. While the ice is still melted, this concept is more energy efficient than Cryobot, because the ice chips are melted inside the insulated interior of vehicle, hence heat does not dissipate to the ambient ice. With a $1 \mathrm{~kW}$ GPHS on the ice probe and a multi-purpose RTG (MMRTG) on the surface module, (Wilcox, et al., 2017) estimate it can reach $10 \mathrm{~km}$ deep in 27 months. A unique advantage of this concept is that the liquid sample can be sent to the surface module for analysis, which allows use of instruments that can be neither miniaturized nor sterilized sufficiently for descent.

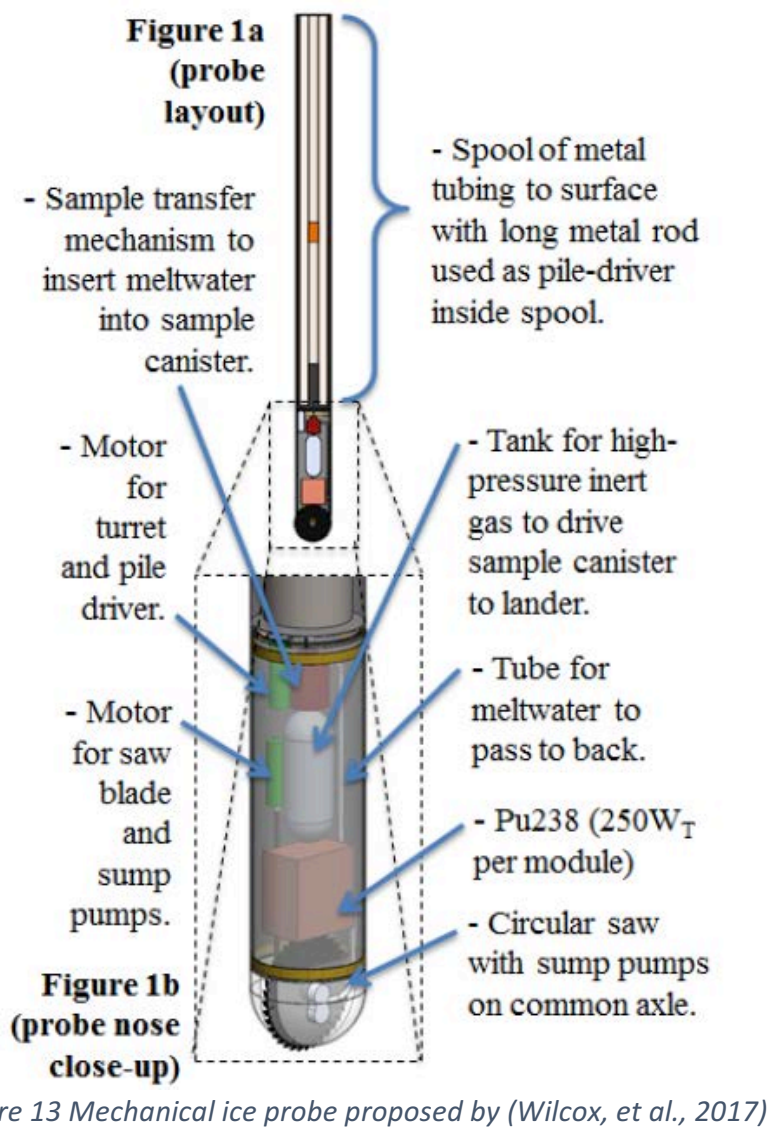




\subsubsection{Comparison with EVE}

The two concepts introduce above share several system-level features with EVE. They have separate descent and surface modules connected by a tether or a tube. They also assume RTG as the power source. Given the difficulty in communication through the ice, as well as the scarcity of sunlight, these features would be natural choices.

The energy efficiency of Eve is orders of magnitude better than thermal and mechanical ice probes because it does not need to melt or mechanically cut the ice. The required energy depends on the dynamic pressure. With our prototype design presented in Section 7, the required energy is $\sim 3 \mathrm{~kJ} / \mathrm{m}$ for $10^{3} \mathrm{~Pa}$ of dynamic pressure and $\sim 6.5 \mathrm{~kJ} / \mathrm{m}$ for $10^{5} \mathrm{~Pa}$. The required energy does not scale linearly with dynamic pressure because the energy needed for inserting and removing ice screw is constant over most of the range. Detailed derivation of the energy estimate is described in Section 4.2.1. Assuming $10 \mathrm{~W}$ is available for mobility, the speed of DM is $\sim 12 \mathrm{~m} / \mathrm{hr}$ at $10^{3} \mathrm{~Pa}$ and $\sim 5.5 \mathrm{~m} / \mathrm{hr}$ at $10^{5} \mathrm{~Pa}$. With this rate, descending $10 \mathrm{~km}$ takes about a month at $10^{3} \mathrm{~Pa}$ and 2.5 months at $10^{5} \mathrm{~Pa}$, if operated continuously.

Highly unknown vent size and dynamic pressure, particularly with the "cryovolcanic" model, are the greatest risk for EVE. Another major shortcoming of EVE is mechanical complexity, which in turn requires a high level of machine intelligence. However, the reduced reliability due to the mechanical complexity can be mitigated by operating multiple DMs.

A unique advantage of EVE is its ability to investigate the interior of vent-conduit system, which itself is a subject of intense scientific interest. The vent-conduit system on Europa, if exists, is considered a potentially habitable zone (Fugueredo, et al., 2003), as shown in Figure 14, and likewise that of Enceladus could be habitable, too. If that is the case, the vent-conduit system could be the most accessible habitable zones on icy moons, from which the first discovery of extraterrestrial life could be made. However, as we will describe shortly in Section 3, the eruption mechanism, which couples with geometry, environment, and habitability of the vent-conduit system, is completely unknown. 
THIN-SHELL MODEL

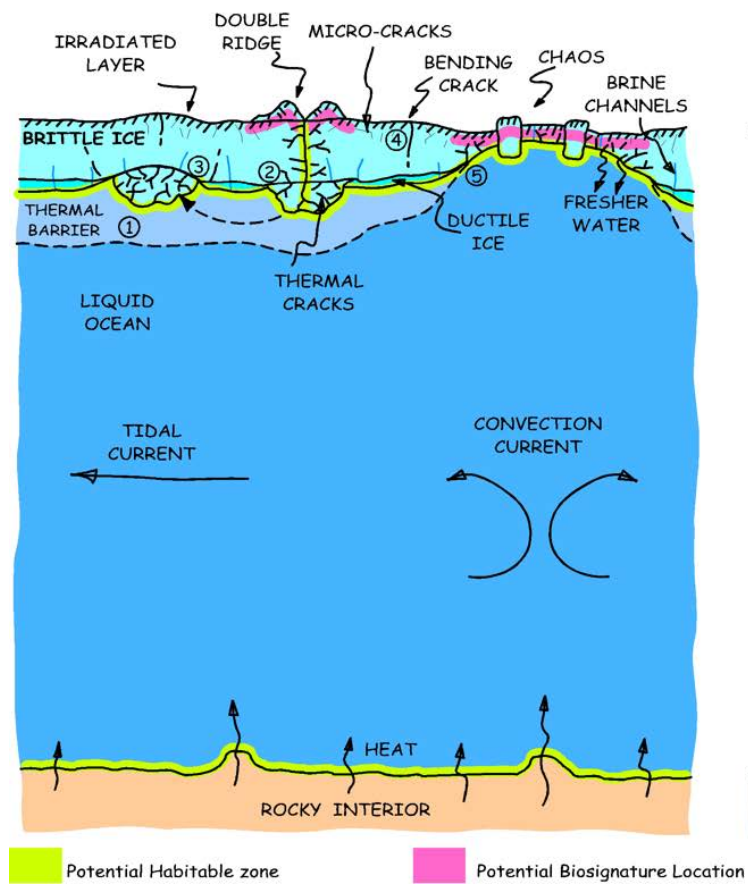

THICK-SHELL MODEL

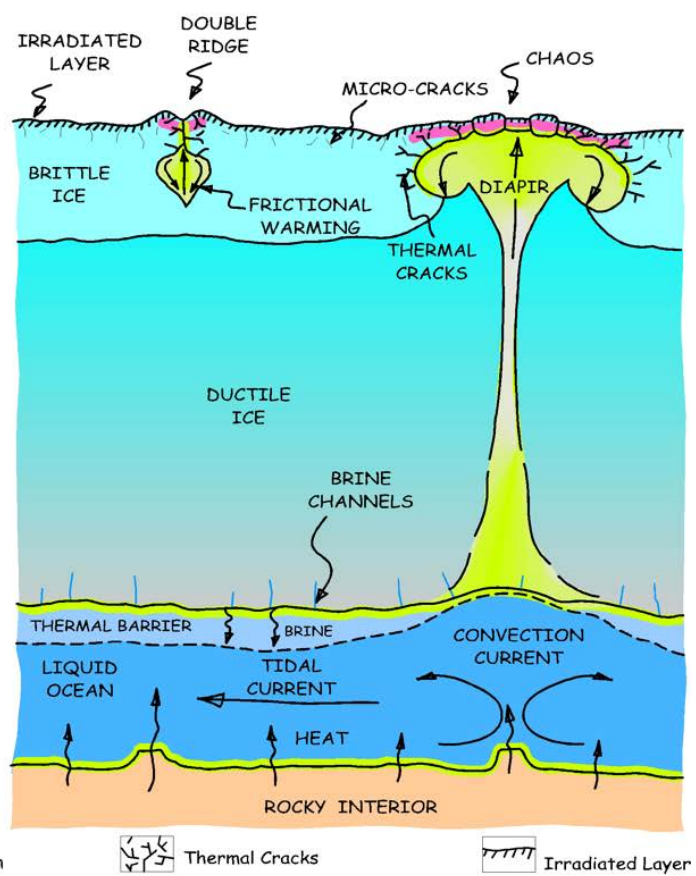

Figure 14 Potential habitable zones (shown in green) of Europa include vent-conduit system, diaper, ice ceiling, and sea floor. EVE particularly targets these zones to maximize the likelihood of the discovery of life. Image taken from (Fugueredo, et al., 2003) 


\section{Characterization of Environment in Enceladus Vents}

\subsection{Overview}

Among numerous unknowns of Enceladus geysers, the one that has the greatest impact on our concept is the eruption mechanism. While many hypothetical eruption models have been proposed, most of them fall into either of two categories: relatively calm "boiling" models and more dynamic "cryovolcanic" models. Very roughly speaking, the "boiling" models assume that liquid water boils into vapor under the surface, while the "cryovolcanic" models assume that the mixture of liquid and gas ascends the conduit and accelerated as the gas expands. More detailed explanations of the two models will be provided shortly. Existing Cassini observations cannot resolve between the two models.

The "boiling" models are more favorable for EVE because it results in a greater vent size and lower dynamic pressure. The worst-case dynamic pressure is $\sim 10^{4} \mathrm{~Pa}$, which is well within the design range of DM. While the vent size is harder to constrain, the "boiling" model gives orderof-magnitude greater estimates than the "cryovolcanic" model in general. For example, Kite and Rubin [2016] suggest "slots" of greater than 1-m in width. EVE is likely feasible with the "boiling" models.

The "cryovolcanic" models are more problematic for EVE. Our preliminary analysis suggests that the dynamic pressure and the vent width could be anywhere between $10^{3}-10^{7} \mathrm{~Pa}$ and $1-30 \mathrm{~cm}$, respectively. The worst-case is beyond the upper limit for DM $\left(\sim 10^{5} \mathrm{~Pa}\right.$ and $\left.\sim 10 \mathrm{~cm}\right)$. While some suggests $10^{7} \mathrm{~Pa}$ dynamic pressure is likely unreasonable for geophysical reasons (D. Hemingway, pers. comm.) and mode detailed analysis could better constrain the worst cases, we do not reject possibilities. Therefore, the feasibility of EVE is undetermined with the current best knowledge with the "cryovolcanic" models.

Our analysis is described below.

\subsection{Observed Data}

The Cassini mission collected a limited amount of data during the performed flyby. An analysis of the chemical composition of the plume based on Cassini Ion Neutral Mass Spectrometer (INMS) data (Waite, et al., 2011), reveals an approximately 8-10 molar\% non- $\mathrm{H}_{2} \mathrm{O}$ fraction dominated by $\mathrm{CO}_{2}$, as well as other volatiles and salts. The Ultra-Violet Imaging Spectrometer (UVIS) shows an expansion jet of the eruptive gases to be consistent with an internal sound speed of between Mach 5 and 8 (Hansen, et al., 2011). Co-analysis of UVIS and Image Science Subsystem (ISS) imagery reveals that the solid:vapor ratio likely is within the $0.35-0.70$ range [Ingersoll and Ewald, 2011], which does not particularly favor either "boiling" (vapor-dominated) or "cryovolcanic" (vapor-poor) models, and a total eruptive mass of order $\sim 200 \mathrm{~kg} / \mathrm{s}$ over the entire system. Gigawatts of thermal emission detected by the Composite Infra-Red Spectrometer (CIRS) reveals elevated temperatures and emissions in the tiger-stripes region in excess of what can reasonable be supplied via conductive losses from erupting materials through the walls of the conduit. Detection of higher temperature emissions very close to the tiger-stripes, by the Visual and Infrared Mapping Spectrometer (VIMS), highlight an unusually-elevated surface temperature 
(Goguen, et al., 2013) of approximately $200 \mathrm{~K}$ (compared with $70 \mathrm{~K}$ typical surface temperature) within a few meters of the tiger stripes. It remains unclear at this stage whether eruptions emanate from point sources along the tiger stripes, or as curtains (Spitale, et al., 2015) [Spitale et al., 2015], or perhaps both, with focusing of non-vapor components in the point sources but a more general diffuse emission of water vapor from along the stripes.

For the worst case cryovolcanic scenarios listed, most of the thermodynamic starting conditions assume a conduit and eruption temperature of $0^{\circ} \mathrm{C}$, i.e. buffered by the latent heat of solidification of water-ice. For boiling models, the temperature profile may be considerably lower.

\subsection{Dynamics Assumptions}

The physics models used to describe "cryovolcanic" models differ from the "boiling" models.

"Boiling models" (e.g., (Kite \& Rubin, 2016)) assume that the thermodynamic starting points of the erupting fluids begin at a higher entropy state (e.g., gas) for the boiling models, and theorize the presence of an interface somewhere between Enceladus' surface and the underground ocean, the liquid surface is exposed to a pressure close to or below the pressure of the triple point of water. Due to the low pressure the water is forced to boil and evaporate, providing the matter expelled from the vents. In this instance, the eruption velocities of different phases (water vapor, non-water vapor, and water solids/liquids) may differ considerably. These models do not easily explain why the sampled data shows high percentage exsolved substances such $\mathrm{CO}_{2}$ and salts that should be either concentrated within or depleted from the erupting materials. Most importantly, they are associated with larger vents, lower eruption pressures and, it turns out, less dynamic pressure.

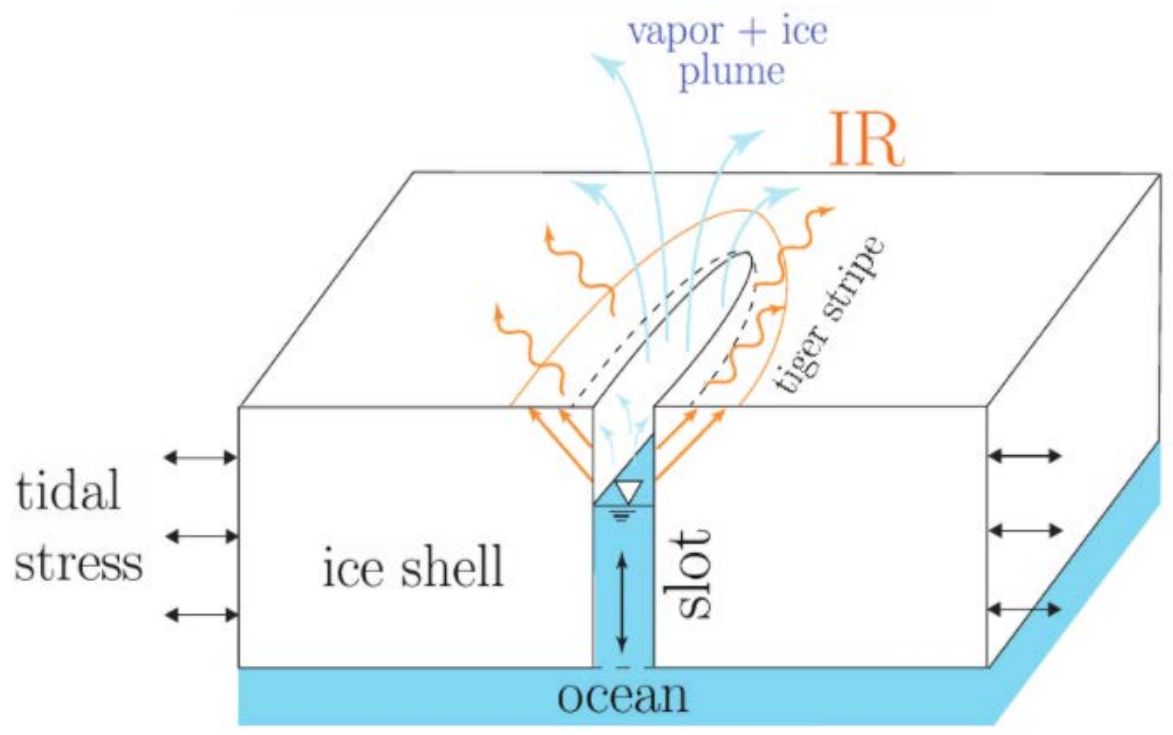

Figure 15 A "boiling" model of Enceladus eruption by (Kite \& Rubin, 2016)

"Cryovolcanic" models (Matson, et al., 2012; Porco, et al., 2014), on the other hand, propose that liquids and dissolved materials ascent in a coupled state, driven by the exsolution and expansion 
of dissolved gases under a negative pressure gradient. Thermodynamically they start their ascent in a low entropy state (liquid water), and the gases, despite being a minor percentage of the mass ratio, eventually massively dominate the volume ratio and continue drag the water particles (containing dissolved materials such as salts) up to the vent. Only later when the static pressure reach the tripe point of pressure the liquid water changes state (the model doesn't take account of any latency in the process nor of any metastable phase that may be expected and become problematic in the development). The ratio of water changed in gaseous and solid state will then depend on the ratio of latent heat of fusion and evaporation so to preserve the local balance of energy.

Due to the presence of a supersonic regime it's likely that a supersonic nozzle exists somewhere within the system, most likely close to the surface, where the sonic condition is achieved. A possible variant of it is described by a parallel wall model in which the flow is kept at a under sonic velocity, in which case the pressure is almost certainly higher, before reaching the speed of sound at the surface and quickly decompressing accelerating into the vacuum of space before reaching a much higher velocity.

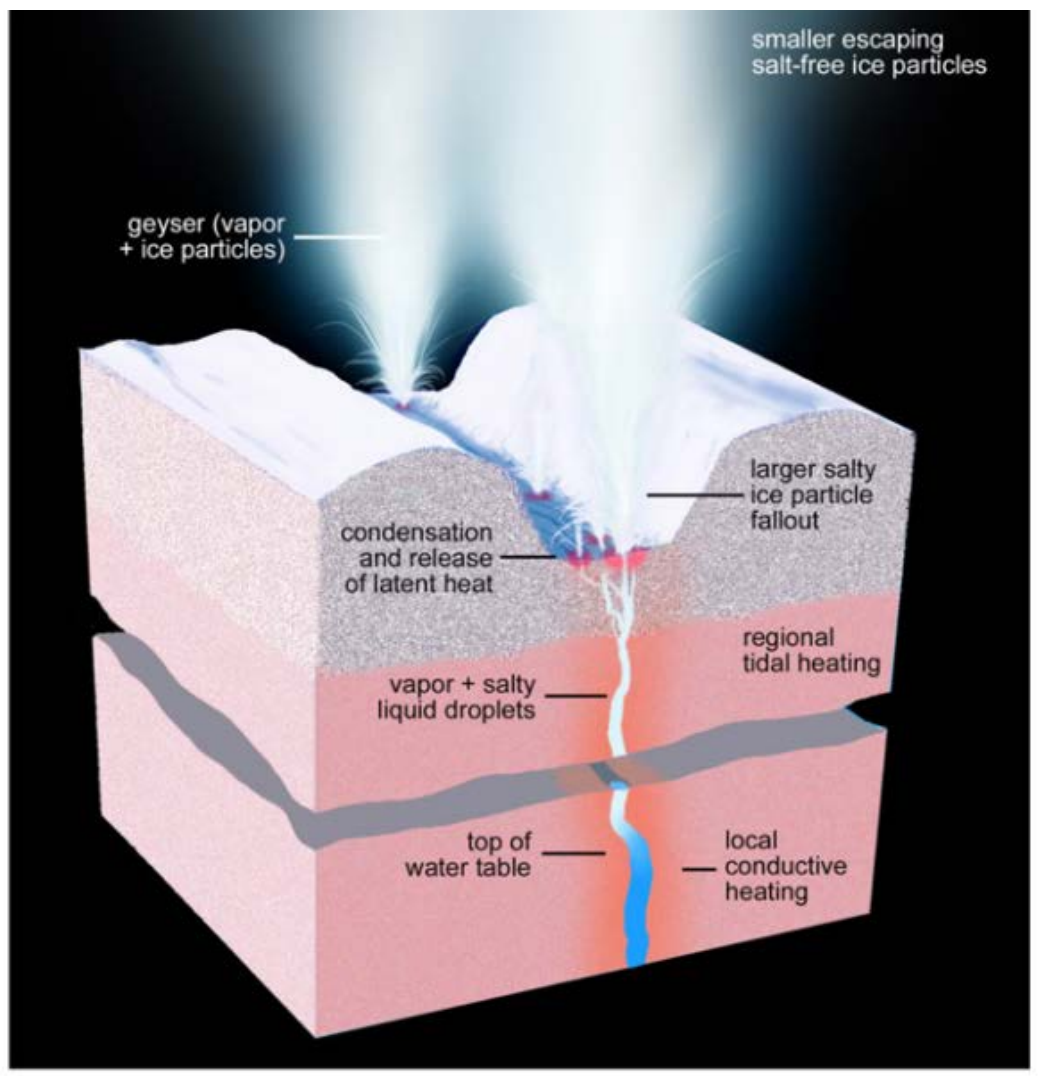

Figure 16 A cryovolcanic model of Enceladus eruption by (Porco, et al., 2014). 


\subsection{Vent dynamic model}

A fluid in motion exerts a pressure that depends both on the static pressure, $p$, and on the pressure caused by the energy of its motion, its dynamic pressure, $q$, where:

$q=1 / 2 \rho u^{2}$,

where $u$ is the velocity and $\rho$ is the density. For high speed systems, however, an alternative formulation can be defined with respect to the speed of sound. By applying the ideal gas law, as well as definitions of the speed of sound and the Mach number, $M$, it can further be shown that:

$q=1 / 2$ rp $M^{2}$,

where $\gamma$ is the ratio of specific heats. We consider three approaches to constraining upper limits to $q$.

Maximum exit velocity: We consider a scenario in which most of the acceleration occurs in the subsurface and water erupts at sub-triple points pressures (vapor + solid). This is most likely where pressures at the exit are low, which is more (although not uniquely) consistent with "boiling" models; Certainly, gas slow would have to dominate. In this case, we use eq. 2, assume $p<611 \mathrm{~Pa}$ and, from studies of the jet structures [1], $5<M<8$.

The ratio of specific heats of a pseudogas mixture, $\nu_{\text {mix }}$, can be determined using adiabatic ratios of the individual components w.r.t. pressure and volume, weighted by their mass fractions, $w$ :

$V_{\text {mix }}=\Sigma_{\mathrm{i}}\left(w_{\mathrm{i}} c_{\mathrm{p}, \mathrm{i}}\right) / \Sigma_{\mathrm{i}}\left(w_{\mathrm{i}} c_{\mathrm{v}, \mathrm{i}}\right)$

Based on results from the Cassini Ion Neutral Mass Spectrometer (INMS), we can assume that a proportion of the plume is exsolvable non-water volatiles, and based on compositions observed by Cassini's E5 and E7 Enceladus fly-bys [2] we consider it reasonable to assume $8 \%$ (molar) $\mathrm{CO}_{2}$ as a proxy for all non-water volatiles. $\mathrm{CO}_{2}$ and $\mathrm{H}_{2} \mathrm{O}$ water vapor at 0 - $\mathrm{C}$ have specific heats capacities at constant pressures of $c_{\mathrm{p}, \mathrm{CO} 2 \mathrm{v}}=800 \mathrm{~J} \mathrm{~kg}^{-1} \mathrm{~K}^{-1}, c_{\mathrm{p}, \mathrm{H} 2 \mathrm{Ov}}=1860 \mathrm{~J} \mathrm{~kg}^{-1} \mathrm{~K}^{-1}$. Specific heat capacity at constant volume, $c_{\mathrm{v}}$, can be derived using the ratios of specific heat at $0 . \mathrm{C}$, $\gamma_{C O 2}=c_{p} / c_{v}=1.31$ and $\gamma_{H 2 O}=c_{p} / c_{v}=1.33$. For water-ice, $c_{p}=c_{v}=2100 \mathrm{~J} \mathrm{~kg}^{-1} \mathrm{~K}^{-1}$. Thus, making no assumptions about the relative quantities of $\mathrm{CO}_{2}$ and $\mathrm{H}_{2} \mathrm{O}$, we find $\gamma_{\text {mix }} \sim 1.05$. Note that there is an inherent assumption that exit velocities are the same for gas and non-gas phases, which is flawed but doesn't make a massive difference to the model.

Application of eq. 2 gives $q<2.05 \times 10^{4} \mathrm{~Pa}$, equivalent to a terrestrial surface wind speed of 185 $\mathrm{m} / \mathrm{s}$ (414 $\mathrm{mph}$ ). This is an over-estimate, as some acceleration will occur below triple point pressures, but given that this is not at the higher end of our dynamic pressure space we do not consider it critical for this study.

Choked eruption: Another estimate can be derived by considering the dynamic pressure at Mach 1, a likely condition at the surface (surface choked) or at the narrowest point of a converging-diverging nozzle (the "throat"). For the same static pressure as above, the maximum value for $q$ would 1-2 orders of magnitude less than that for the above scenario using eq. 2 (The difference corresponds to the "Max v" line and the colored lines in Figure 17-b. 
However, in this case it is useful to determine the solution semi-analytically for higher unknown pressures using eq. 1 , as $u$ is known to be equal to $C$, the mixed-phase sound speed velocity, an approximation for which is (Lorenz, 2002):

$C=(A B)^{-1}$

$A=\left[\Sigma_{\mathrm{i}}\left(\phi_{\mathrm{i}} \rho_{\mathrm{i}}\right)\right]^{0.5}$

$B=\left[\Sigma_{\mathrm{i}}\left(\phi_{\mathrm{i}} / \rho_{\mathrm{i}} c^{2}\right)\right]^{0.5}$,

where $c$ and $\rho$ are the speed of sound and densities of the individual components.

As previously, we assume that $\mathrm{CO}_{2}$ drives ascent, and that it exsolves from the $\mathrm{H}_{2} \mathrm{O}$ in equilibrium according to Henry's Law $\left(\mathrm{H}^{\mathrm{cp}}=0.034 \mathrm{~mol} \mathrm{~L}^{-1} \mathrm{~atm}^{-1}\right)$. Water remains in a liquid state (>611 Pa), consistent with adiabatic liquid ascent (cryovolcanism). Results are given in Figure 17 for a range of pressures and $\mathrm{CO}_{2}$ starting molar fractions.

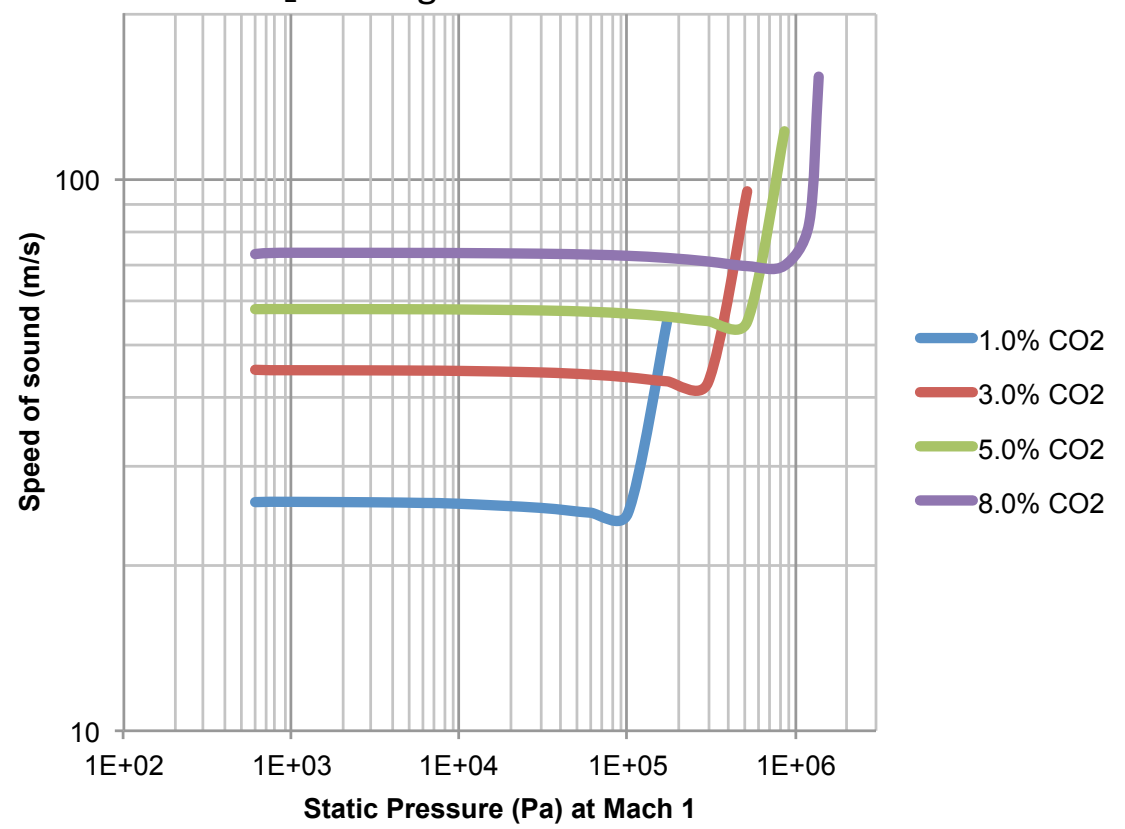

(a) Speed of sound 

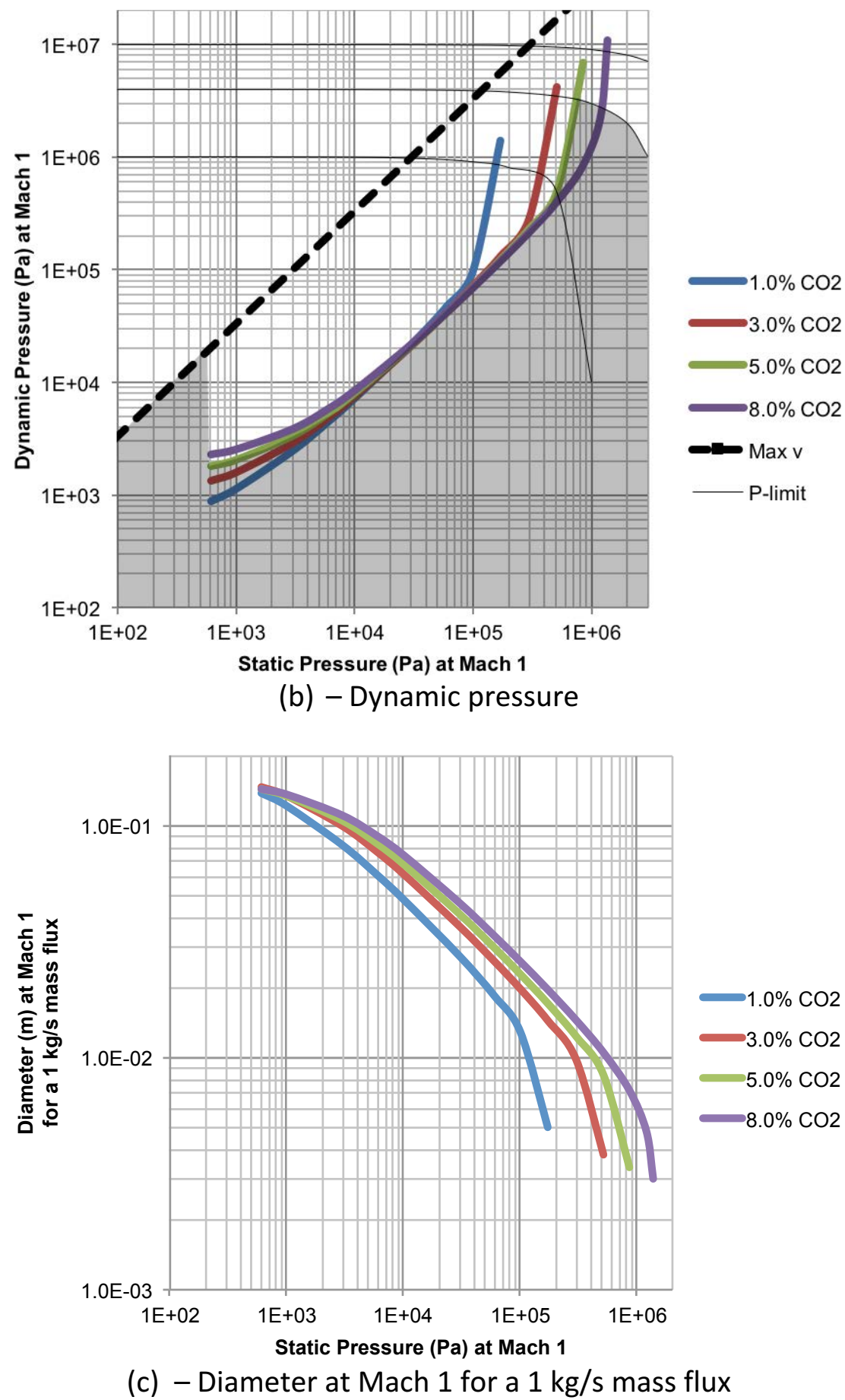

Figure 17 (a) Sound speed, (b) dynamic pressure and (c) diameter for a CO2-driven eruption at Mach 1 (surface choked or in nozzle throat). $\mathrm{CO} 2$ is used as a proxy for all volatiles, and the lowest and highest values (1 and 8 molar\%) both seem unlikely, given that $\mathrm{CO} 2$ has a considerable molar weight. Upper limit at $\rho=920 \mathrm{~kg} \mathrm{~m}-3$ (ice density), lower limit at $p=610 \mathrm{~Pa}$ (triple point of water). Results for an upper Mach 8 velocity (marked MPV) are also included. The diameter is for a radially-symmetric conduit with an eruptive mass flux, $\dot{M}$, of $1 \mathrm{~kg} / \mathrm{sec}$; From conservation of mass $(\dot{M}=$ rho . u . A), diameter scales with $\sqrt{M}$ for different mass fluxes. In (b), we assumed that the ocean is at $4 \mathrm{MPa}$, and that there is 5 molar\% of CO2. Anywhere that is shaded grey is "plausible". To the right of 600 Pa static pressure is all cryovolcanic conditions at Mach 1 (where the dynamic pressure is highest). To the left of $\sim 600 \mathrm{~Pa}$ is all boiling models. 
In this scenario, the dynamic pressure in the throat approximates the static pressure. Eruptions may only occur if the mean fluid density integrated from ocean to surface is less than the mean crustal density, i.e. the liquid is hydraulically buoyancy, and so results with $\rho>920 \mathrm{~kg} \mathrm{~m}^{-3}$ are rejected (curves are truncated); Further analysis will rein this in further. The highest dynamic pressures ( $>1 \mathrm{MPa}$ ) occur with high $\mathrm{CO}_{2}$ contents and static pressures (also $1 \mathrm{MPa}$ ), equivalent to a terrestrial ground wind speed of $1290 \mathrm{~m} \mathrm{~s}^{-1}$. Such values may also be unrealistic if they exceed ocean pressures, or if exsolution of available $\mathrm{CO}_{2}$ is suppressed (likely) due to supersaturation or formation of carbonic acid, which will be considered in future analyses. Furthermore, we can also relate static pressure to conduit cross-sectional area as a function of mass flux (Figure 17 bottom), by considering conservation of mass $(\dot{M}=\rho u A)$, where $A$ is the cross-sectional area (N.B. $A=\pi r^{2}$, where $r$ is the radius). Note that for fissure eruptions the fissure width is considerably smaller.

Supersonic nozzle flow: (Mitchell, 2005) proposed that abrasive erosion of conduit walls in volcanic eruptions would, given as a sufficiently long-lived eruption, as seems plausible on Enceladus, result in the fluid dynamic conditions (e.g. a de Laval nozzle) necessary to facilitate descent of the throat into the subsurface and enable supersonic flow the surface. From conservation of mass, if we assume isentropic flow, which is reasonable for a supersonic jet, through an ideal de Laval nozzle, it has been shown (Liepmann \& Roshko, 2002) that: 


$$
\begin{aligned}
& \left(\frac{A}{A^{*}}\right)^{2}=\frac{1}{M^{2}}\left[\frac{2}{\gamma+1}\left(1+\frac{\gamma-1}{2} M^{2}\right)\right]^{(\gamma+1)(\gamma-1)} \\
& \frac{A^{*}}{A}=\frac{\rho u}{\rho^{*} u^{*}}=\frac{\left[1-\left(\frac{p}{p_{0}}\right)^{(\gamma-1) / \gamma}\right]^{\frac{1}{2}}\left(\frac{p}{p_{0}}\right)^{1 / \gamma}}{\left(\frac{\gamma-1}{2}\right)^{(1 / 2)}\left(\frac{2}{\gamma-1}\right)^{(1 / 2)(\gamma+1) /(\gamma-1)}} .
\end{aligned}
$$

where $A$ is the conduit cross-sectional area, and superscript * refers to the condition at $M=1$.

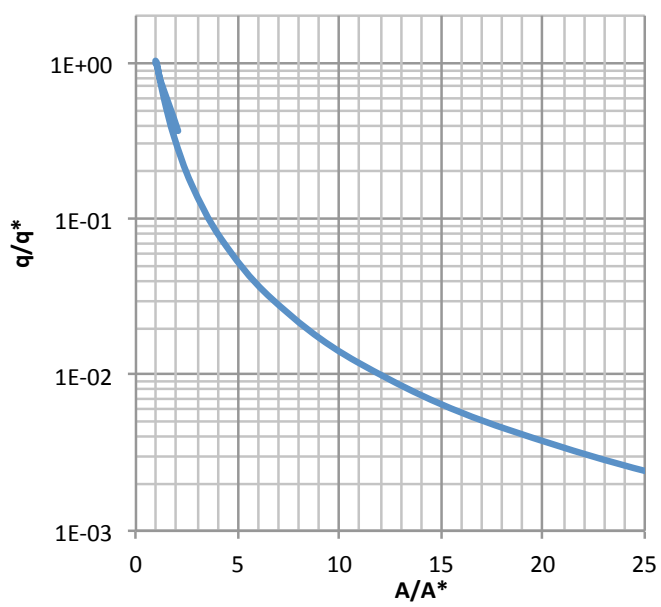

Figure 18 Relationship between dynamic pressure and conduit cross sectional area in a de Laval nozzle assuming isentropic flow and negligible phase changes, both w.r.t. the condition at Mach 1 ( $^{*}$ annotated). The short tail is for elevations below the throat, where flow is subsonic.

Hence it is possible to track how pressure, Mach number and cross-sectional area change relative to their values at the throat, which was determined above (Figure 17). Dynamic pressure is presented, relative to the throat as a function of expanding vent/jet angle (Figure 18), demonstrating that the throat is likely to be the location of peak pressure (it may be a few percent higher within a few vent radii of the surface). It is clear that dynamic pressure is at its peak in the throat at Mach 1. Note that this analysis does not consider the consequences of phase change after this condition, which is inevitable during decompression through the triple point.

Stagnation pressure: Finally, we can demonstrate an additional limit to dynamic pressure on the basis of conservation of energy, thus:

$T \mathrm{~d} s-P \mathrm{~d} v+u \mathrm{~d} u-g \mathrm{~d} z-\Sigma_{\mathrm{i}} \mathrm{d} w_{\mathrm{i}} \mathrm{d} h_{\mathrm{i}}=0$, (6) where $T$ is the temperature, $s$ is entropy, $v$ is specific volume, $g$ is the gravity $(-0.113 \mathrm{~m} / \mathrm{s}), w_{i}$ is the mass fraction of a material $i$ changing state, and $h_{\mathrm{i}}$ is the enthalpy of that change of state. We know that $\mathrm{d} v=1 / \mathrm{d} \rho$. We can assume that $g \mathrm{~d} z$ small and positive, that velocity is 0 at source and positive in the vent, that $d s$ is positive (second law of thermodynamics), and that all changes of state are endogenic hence $\Sigma_{\mathrm{i}} \mathrm{d} w_{\mathrm{i}} \mathrm{d} h_{\mathrm{i}}$ is positive, thus:

$P^{*}-P_{\mathrm{i}}<u \mathrm{~d} u \mathrm{~d} \rho$ where i refers to the condition in the source reservoir. Finally, integrating the right-hand-side (assuming a fairly linear correlation between $\mathrm{d} u$ and $\mathrm{d} \rho$ which should be reasonable to $1^{\text {st }}$ 
order), assuming that velocity is 0 at source, and substituting in for the definition of dynamic velocity we find that:

$P^{*}+q<P_{\mathrm{i}}$

$P_{\mathrm{i}}$ represents the concept of stagnation pressure in fluid dynamic texts. Thus, we demonstrate that the concept of stagnation pressure does apply for this situation. Upper limit curves due to the stagnation pressure concept are added as thin, dark lines fin Figure 17(b); Note that the 10 MPa pressure line is likely unreasonable for geophysical reasons (D. Hemingway, pers. comm.), but demonstrating this was beyond the scope of what was plausible in this study.

Furthermore, we can define the effective stagnation pressure, $P_{\mathrm{s}, \mathrm{eff}}$ as:

$\left(P_{\mathrm{s}, \mathrm{eff}}-P_{0}\right) \mathrm{d} v=-T \mathrm{~d} s+g \mathrm{~d} z+\Sigma_{\mathrm{i}} \mathrm{d} w_{\mathrm{i}} \mathrm{d} h_{\mathrm{i}}-\mathrm{Q}^{\prime}(9)$.

where $Q^{\prime}$ represents loss of energy from the system (most likely by conduction), and use this to estimate how far below the stagnation pressure the sum of $P_{s}+q$ is. The worst-case scenario is one in which the flow is isentropic $(T \mathrm{~d} s=0 \mathrm{~kJ} / \mathrm{kg})$, ascent starts at the top of the deepest conceived ocean ( $g \mathrm{~d} z=4.62 \mathrm{~kJ} / \mathrm{kg}$ ). It is more difficult to put universally applicable limits on $P_{0}$ and $\mathrm{d} w_{\mathrm{i}}$, but we hope to do more in the future. If we assume that solubility is well described by Henry's Law, and that $\mathrm{CO}_{2}$ is at saturation at source, we can estimate an upper limit for $\mathrm{d} w_{\mathrm{i}}$ as a function of $P_{0}$, but we can't escape the possibility that the lower limit for $\mathrm{d} w_{\mathrm{i}}$ is negligible.

Summary: Taken together, there exists a very wide range of dynamic pressures to account for. Every curve on Figure 17 should be considered an upper limit, and furthermore a conservative one. Within that context dynamics pressures as great as $\sim 9 \mathrm{MPa}$ must be considered, although if we accept the assertion of $\mathrm{D}$. Hemmingway (pers. comm.) that $10 \mathrm{MPa}$ is far too great an ocean pressure then 3-4 MPa may be more reasonable. This is considerable, and the equivalent to a surface wind on Earth of around $2500 \mathrm{~m} / \mathrm{s}$, which sounds extreme. It is, of course, also considerably less than in most terrestrial volcanic eruptions. For the Kite and Rubin "boiling" model, we would anticipate far lower, trivial dynamic pressures.

\subsection{Diameter estimates}

The cross-sectional areas for "boiling" models are generally quite high. (Kite \& Rubin, 2016) suggest "slots" of greater than 1-m in width, which is not considered problematic. However, for cryovolcanic models, especially those with high pressure surface conditions, vent diameters tend to be way smaller. From conservation of mass, the mass flux, $\dot{M}=\rho u A$, and so if we assume an ideal gas we can determine the cross-sectional area and hence the diameter as a function of mass flux. This is illustrated in (Figure 17) for a $1 \mathrm{~kg} / \mathrm{s}$ vent. Note that the ideal gas equation is an imperfect predictor of the relationship between pressure and density, but that in this case it will give higher density for a given pressure than alternative formulations, and hence predicts the smallest (i.e., worst-case) cross sectional areas. As diameter is proportional to the square root of the mass flux, a perhaps more reasonable flux through a vent is $2.5 \mathrm{~kg} / \mathrm{s}$, based on observations by Porco et al. (pers. comm.) who identify of order 100 the number of discrete point sources within the $\sim 500 \mathrm{~km}$ of tiger stripes. Hence, within the parameter space being explored, we find $10-300 \mathrm{~mm}$ is plausible, and selection of the most active vents (in terms of mass flux) could give larger diameters yet. Note, however, that this is a lower limit range, 
and we suspect that the $\sim 10 \mathrm{~mm}$ end is implausible; It's just that we haven't had the time to more fully explore the problem, which remains unpublished. We decided, in absence of a more refined model, to keep the $100 \mathrm{~mm}$ diameter limit as reference.

\subsection{Nozzle shape estimations}

The models presented do not make detailed predictions of the conduit and vent geometry, beyond the de Laval nozzle structure shown in Figure 18. The worst-case scenario is represented by a single very thin fracture all the way along the stripes. That case has yet to be proved impossible but it seems very unlikely on the basis of thermal efficiency; On Earth in silicate volcanic eruptions, materials freeze on the walls of such narrow conduits, blocking them, focusing erupting materials through the widest points which eventually circularize. This seems likely to occur on Enceladus. If a true fissure process exists on Enceladus, it is therefore likely to be transient and short-lived (irrelevant given the continuous activity observed), or the result of a "boiling" model [e.g. Kite and Rubin, 2016].

\subsection{Suggestions for future study to better constrain key parameters}

Further study of Cassini data could better constrain parameter estimates of each model. For example, resolving between discrete "point" sources and more extensive "fissure" eruptions may allow certain models to be tested. However, in order to resolve between "boiling" and "cryovolcanic" models, additional orbital observations are likely needed. For example, the "boiling" model of Kite and Rubin [2016] seems to require a "slot" geometry, i.e. a fissure, while the cryovolcanic models are not as thermally stable as a fissure. Observing mass flux with carefully controlled orbital geometries, as well as the solid/vapor ratios along the tiger stripes, preferable at $<10 \mathrm{~km}$ would be valuable.

In the near-term, there is still much we can do with existing data. For example, characterization of the upper limits of ocean pressure and volatile fractions could put additional constraints on dynamic pressure in the vent. Multi-species thermochemical/exsolution models would allow detailed tests of current conceptual models for ascent and eruption, and may allow for enhanced interpretation of plume mass spectrometric results.

Capturing the dynamic conditions at as high a spatial resolution as possible within individual jets should allow testing between different models, as well as constraints upon those individual models. This will require careful instrument design and selection, and very specific geometric constraints on observations. However, if a cryovolcanic model is determined to be true, additional constraints will need to be determined, as the range of exit pressures (and hence area and dynamic pressure) are extremely large.

Very high resolution imaging combined with extremely sensitive passive emission spectrometry at submm/THz wavelengths at optimized phase angles may give the highest potential for resolving this. The former allows analysis of the structure of the solid particle components of the plume, much like with Cassini ISS. The latter allows simultaneous characterization of optical depth of different polar molecules in the vapor phase (especially water), as well as some 
constraints on pressure and temperature; with sufficiently high resolution observations it may be possible to truly constrain the fluid mechanics of observed jets; The alternative, UV spectroscopy (as used by Cassini UVIS), struggles to resolve the effects of temperature and pressure, works in a transmissive mode which requires solar or stellar occultations and thus further constrains observation geometry.

Further constraining the chemistry of the plumes, as proposed by ELF, will also be beneficial if there are sufficient advances in thermochemical studies to (i) make a reasonable range of predictions of ocean chemistries, and (ii0 allow testing of how different eruption modes modulate input chemistries. 


\section{Trade Studies}

We performed trade analyses in six domains: mobility, power, tether, autonomy, perception, and science instrument systems.

\subsection{Mobility System}

\subsubsection{Mobility type}

We first performed a high-level trade-off on down-the-vent mobility. We considered the following three options:

1. No control (falling)

2. 1-D mobility (rappelling)

3. 2-D mobility (climbing)

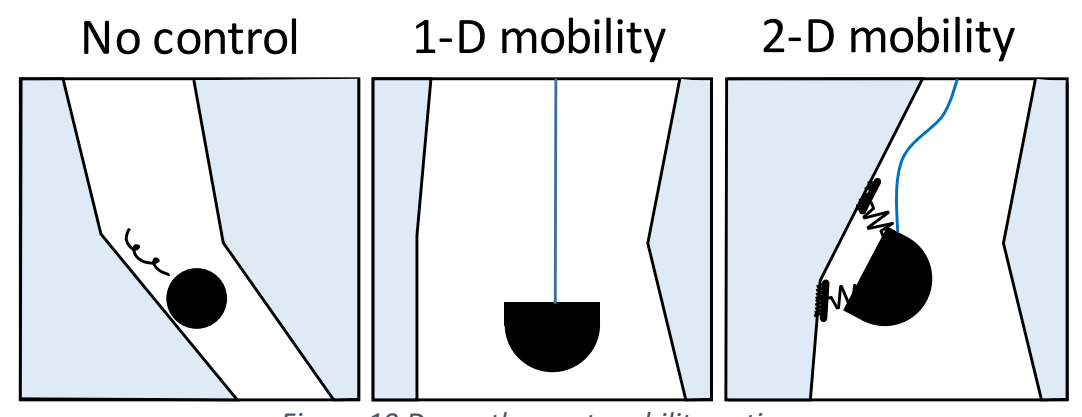

Figure 19 Down-the-vent mobility options

In the "no control" option, DM goes down the vent by solely depending on the gravitational force, with no controllability of its position and speed (e.g., drop sphere). The "1-D mobility" option also solely depends on the gravitational force to go down, but DM's depth and descent speed is controllable through unreeling or reeling in tether. In the "2-D mobility" option, DM can traverse on the vent walls through a mechanism to attach to the surface.

We conclude that 2-D mobility is the only option that can be feasible. The table below summarizes the analysis result. The details of the analysis are described in the rest of this section.

Table 1 Comparison of high-level down-the-vent mobility options

\begin{tabular}{|c|c|c|}
\hline & Can descend Enceladus vent? & Other risks \\
\hline No control (falling) & No & Communication \\
\hline 1-D mobility (rappelling) & No & $\begin{array}{l}\text { Cannot go through non- } \\
\text { straight path }\end{array}$ \\
\hline 2-D mobility (climbing) & Possibly & $\begin{array}{l}\text { Mechanical complexity, } \\
\text { needs intelligent software } \\
\text { capability }\end{array}$ \\
\hline
\end{tabular}




\subsubsection{Feasibility of the "no-control" (falling) option}

The "no control" option (falling) is infeasible in Enceladus vents even with the most optimistic estimate of the dynamic pressure, due to Enceladus's weak gravity $(0.011 \mathrm{~g})$. On Europa, it is potentially feasible because of the significantly greater gravity $(0.13 \mathrm{~g})$, but the dynamic pressure and vent geometry is unconstrained and hence it is not possible to quantitatively assess the feasibility.

Our analysis is based on assumptions that 1$) \mathrm{DM}$ is a sphere with $5 \mathrm{~cm}$ radius $(r), 2) \mathrm{DM}^{\prime}$ 's density $(\rho)$ is $2,000 \mathrm{~kg} / \mathrm{m}^{3}$ (twice of liquid water), and 3) the dynamic pressure $\left(P_{\mathrm{D}}\right)$ is $1000 \mathrm{~Pa}$ (the most optimistic estimate in the "cryovolcanic" model). Given that Enceladus's surface gravity $(g)$ is $0.11 \mathrm{~m} / \mathrm{s}^{2}$, the upward force provided by the gravity is:

$$
F_{\text {Down }}=\frac{4}{3} \pi r^{3} \rho g=0.24 \mathrm{~N} .
$$

The upward force provided by the flow is:

$$
F_{\mathrm{Up}}=\pi r^{2} C_{D} P_{D}=1.9 \mathrm{~N} \text {, }
$$

where $C_{D}=0.47$ is the drag coefficient of a sphere. The upward force is significantly greater than the upward force, hence the drop sphere cannot go down the vent.

In order to make the "no control" option feasible, it has to be either: a) greater than $~ 80 \mathrm{~cm}$ in radius or b) heavier than $\sim 30,000 \mathrm{~kg} / \mathrm{m}^{3}$ in density. a) is not viable given that the lower bound on the vent width is $\sim 10 \mathrm{~cm}$. b) is not possible as it is denser than the densest element (Osmium).

\subsubsection{Feasibility of the "1-D mobility" (rappelling) option}

Rappelling is not feasible on Enceladus as it solely depends gravity to provide downward force (thus the same argument as drop package applies). It could be a feasible option on Europa, but again quantitative feasibility study is not possible due to unconstrained vent conditions. It also has difficulty in going through non-straight vent.

\subsubsection{Feasibility of the "2-D mobility" (climbing) option}

Climbing has a potential of being able to go down the vent because 1) it can mechanically provide additional downward force and 2) it can navigate through a non-straight path. The feasibility of this option primality depends on 1) the maximum downward force that the attachment mechanism can provide, and 2) the availability of power. The major risks of 2-D mobility include mechanical complexity and the need for intelligent autonomy capability to control the complex mechanism. Further analysis of 2-D mobility will be performed in the rest of this section. The autonomy system will be discussed in Section 4.4 .

\subsubsection{Attachment Mechanism}

As mentioned previously, the major challenge for 2-D mobility is the significant uncertainty in the condition of vents, particularly in the dynamic pressure. We identified multiple attachment mechanisms, and assessed their robustness to the uncertainties.

We considered the following attachment mechanisms: 
1. Ice screw: An anchoring device commonly used for ice climbing and crevasse rescue, shown in Figure 20. Typically, ice screws present a hollow center that reduce the amount of energy required in the installation, as well as three to four teeth tips to start the hole and a triangular thread. The shape allows the thread to self-feed itself once the first thread is fully inside the ice, which allows to release the preload required to start the hole. Ice screws are available in different length and are typically rated for a max load of $10 \mathrm{kN}$ (Beverly \& Stephen , 2009).

2. Microspine gripper: Initially developed at Stanford (Asbeck, et al., 2006) and later at JPL (Parness, 2011), the micro-spines gripper uses numerous tiny spines to engage asperities on the surface. It has been successfully applied to LEMUR rock climbing robots (Parness, et al., 2012), and is planned be used in the proposed Asteroid Redirect Mission to grasp and secure a boulder (Figure 21).

3. Cam/wedging: Pushes against opposing walls in the vent to create a hold using an overcenter mechanism.

4. Archimedes screw: Uses two rotational inputs to screw itself down the vent. It has been used for screw propelled vehicles to drive on soft surfaces including snow.

5. Melt anchors: Uses heat to creates a hole on the ice, which the element itself is inserted and act as anchoring (Figure 22). Generally, to be effective it requires a combination of several probes to establish a single grip. The technology has been developed for establishing a stationary grip for icy moon in low gravity environments, such as a surface walking robot for Europa.

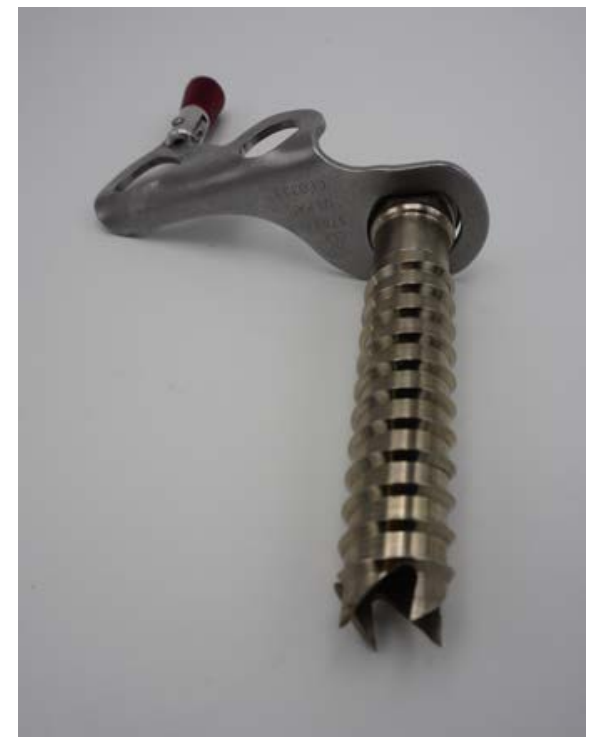

Figure 20 Ice screw 


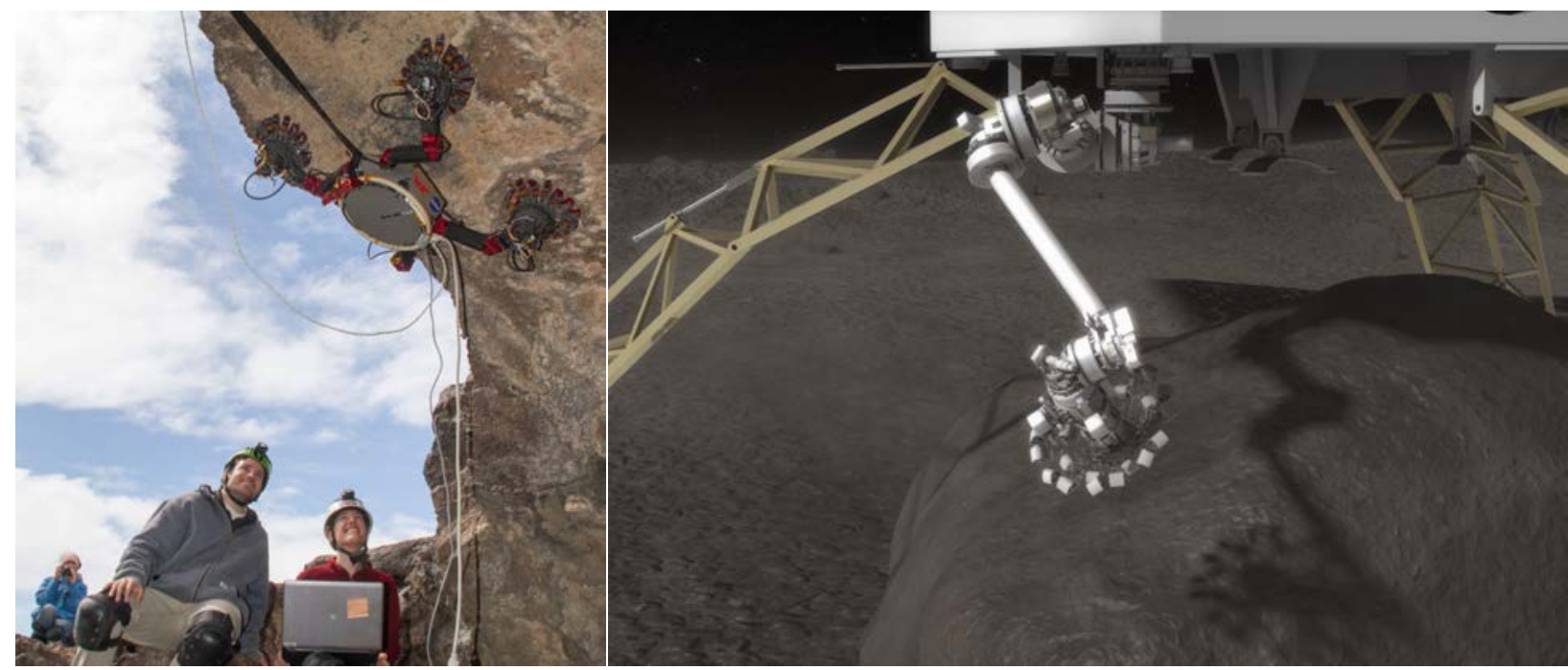

Figure 21 Microspine grippers used for LEMUR climbing robot (left) and the Asteroid Redirect Mission (right)

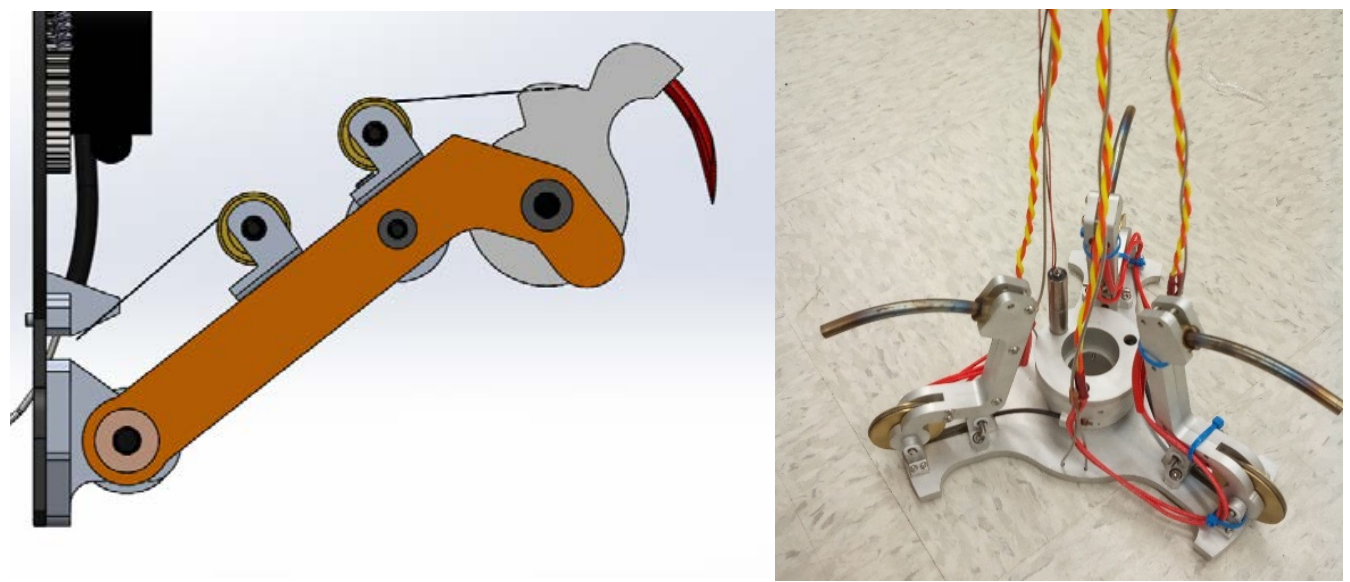

Figure $22 \mathrm{JPL}$ 's melt anchor prototype (left) and a gripper with three melt anchors (right)

These options were compared by five criteria as listed below. The first four is the robustness to uncertainties in the environment of the vent-conduit.

a) Resistance to dynamic pressure: As explained in Section 3, the dynamic pressure in the vent is highly uncertain. The ability to tolerate extremely high dynamic pressure is important.

b) Robustness to unknown mechanical properties of ice: The state of ice can range from pristine ice (strongest) to powdery snow (weakest), and the mechanical properties (e.g., strength) varies significantly. The mechanical properties of ice in the vent is unknown. Therefore, the attachment mechanism should work over a wide range of ice state.

c) Robustness to unknown geometry of vent: The width of the vent is not only unknown but it could vary significantly along the depth. Therefore, the attachment mechanism should work with a wide range of vent width.

d) Robustness to unknown surface roughness: The smoothness of surface of the vent wall is unknown. The attachment mechanism should robustly work on both smooth and undulated surfaces. 
e) Energy/resource required: In addition to the energy consumption, this criterion involves the required supporting mechanism such as robotic limbs.

The five attachment methods are compared by the Pugh matrix, in which options are scored by each of the criteria and ranked by the weighted sum of the scores (Burge, 2009). Its objective is to evaluate a number of design candidates leading ultimately to which best meets a set of criteria. The Pugh matrix is given below. The scores are determined through an intensive discussion by the study team, including two experienced mechanical engineers (Co-I Parness and Co-I Carpenter) and a scientist specialized on ice properties (Co-I Curtis).

\begin{tabular}{|c|c|c|c|c|c|c|c|}
\hline Design & Factor & $\begin{array}{l}\text { Dynamic } \\
\text { Pressure }\end{array}$ & $\begin{array}{l}\text { Properties of } \\
\text { the Ice }\end{array}$ & $\begin{array}{l}\text { Geometry of } \\
\text { the Conduit }\end{array}$ & $\begin{array}{l}\text { Surface } \\
\text { Roughness }\end{array}$ & $\begin{array}{l}\text { Energy/Resou } \\
\text { rces Required }\end{array}$ & Totals \\
\hline & Weight & 5 & 5 & & & 5 & \\
\hline Ice Screw & & 8 & 7 & 10 & 9 & 4 & 190 \\
\hline $\begin{array}{l}\text { Microspine } \\
\text { Gripper }\end{array}$ & & 6 & 3 & 10 & 2 & 6 & 135 \\
\hline Cam/Wedging & & 7 & 5 & 5 & 7 & 7 & 155 \\
\hline $\begin{array}{l}\text { Archimedes } \\
\text { Screw }\end{array}$ & & 8 & 6 & 6 & 8 & 8 & 180 \\
\hline Melt Anchors & & 8 & 7 & 10 & 9 & 3 & 185 \\
\hline & & & & & & & \\
\hline
\end{tabular}

Rank each design in each category. 1 is the worst, 10 is the best. The weights determine the relative importance of each aspect.

\section{Hollow Interior Penetrates Ice} Single Wall Capable

$>4$ Actuators required for movement (Assumes limbed muti DOF)

Figure 23 Pugh matrix for comparing attachment mechanisms

The highest ranked mechanism is ice screw, while Archimedes screw and melt anchors result in comparable scores. Ice screw is chosen as the baseline in our concept, and given further investigations in the rest of this study. While Archimedes screw and melt anchors are also promising, we do not perform detailed investigation of these mechanisms due to the lack of time and resource of the study.

The justifications of the scores in the Pugh matrix is given below.

\subsubsection{Dynamic pressure}

Ice screw and melt anchors can make the strongest anchor because it deeply penetrates into the ice. Archimedes screw can have a hollow interior and mitigate the upward force by the flow, thus it is given the same score as ice screw and melt anchors. 


\subsubsection{Properties of ice}

Ice screw, Archimedes screw, and melt anchor are less sensitive to the properties of ice than the other options because they penetrate ice. We give a slightly smaller score to Archimedes screw than ice screw and melt anchor due to the relatively shallow depth of penetration.

\subsubsection{Geometry of the vent}

Cam/wedging and Archimedes screw must be in contact with opposing walls in order to be operational. As a result, they require relatively accurate prior knowledge on the width of the vent, and have difficulty in adapting to varying vent width. On the other hand, the other three mechanisms only have to be in contact with a single wall, hence significantly more robust to the uncertainty and variation of the vent width.

\subsubsection{Surface roughness}

While the surface state of the ice walls in the vent-conduit system is unknown, the surface is most likely smooth as the weak hoar frost layer, which often develops on ice cave walls on Earth, is blown away by the strong flow. Microspine grippers perform poorly on a smooth surface. On the other hand, ice screw and melt anchor can make a strong anchor on a smooth ice surface.

\subsubsection{Energy/resource required}

Ice screw, microspine gripper, and melt anchor are given low scores because they require at least four multi-DOF, fully actuated limbs. Ice screw and melt anchor need extra energy for drilling and melting ice, respectively.

\subsection{Power System}

\subsubsection{DM Energy Requirement}

We next make a first-order estimate of the energy requirement of DM. This is in part a preparation for the power system trade discussed in the next subsection. Based on the mechanical system trade study, we assume DM is equipped with four limbs and ice screw end effectors. Here we focus on the energy requirement for the mobility. Heating energy can be partially provided by RHU (radioisotope heating unit). We do not include energy requirement for instruments as it varies between different types of DM. Therefore, the estimate in this section must be viewed as an optimistic one, although mobility system is likely to dominate the energy consumption in most types of DMs.

DM is locomoted by repeating the following motions. (Also see Figure 24.)

1. Disengage the ice screw end effector of one limb, push the end effector by extending/contracting the limb, and engage the end effector again

2. Repeat the same for the other three limbs

3. Push the body forward by contracting the front limbs and extending the rear limbs. Based on our prototype design described in Section 7, we assumed the stroke length of the limbs is $15 \mathrm{~cm}$. 


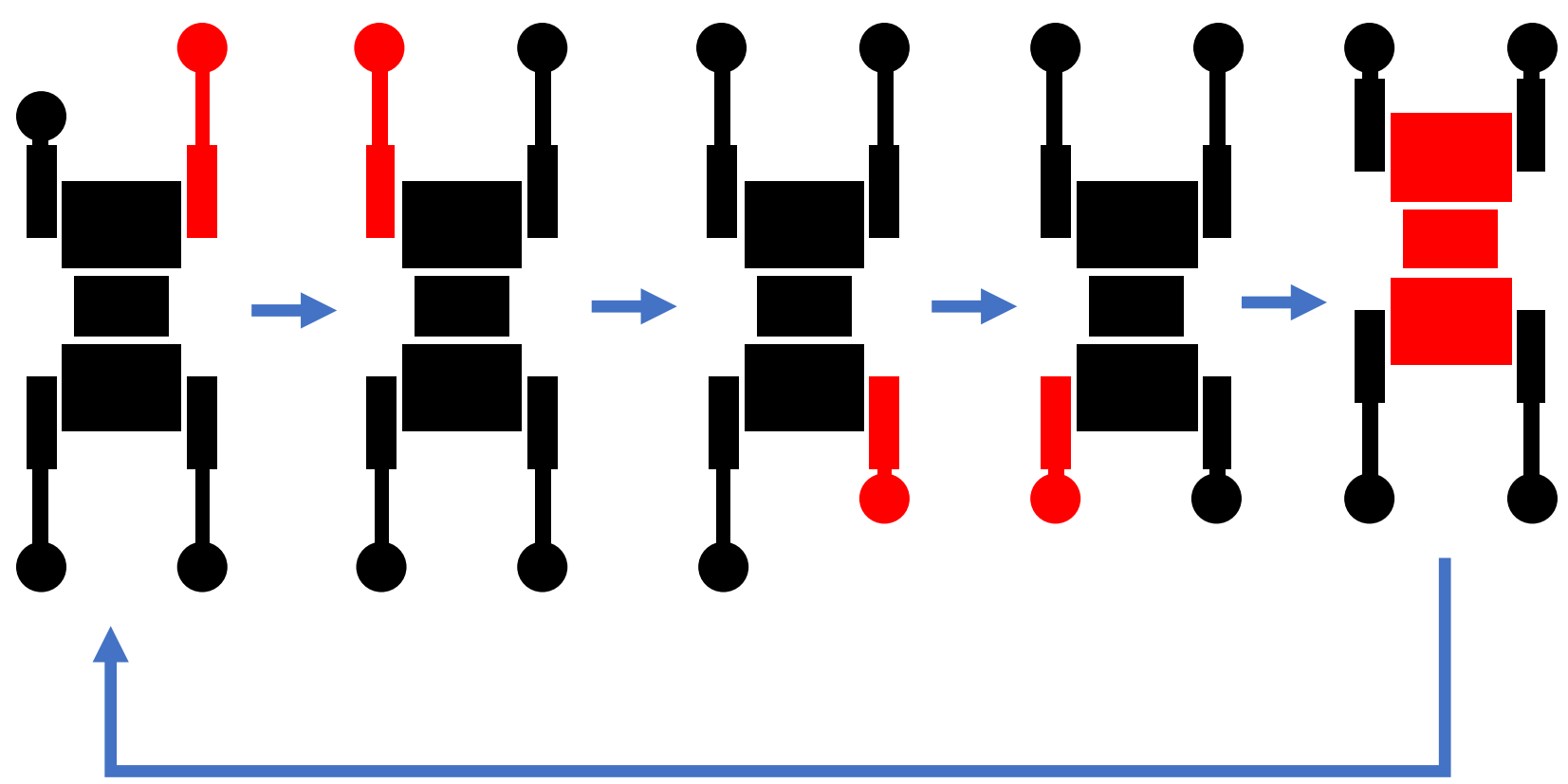

Figure 24 DM's gait

Energy is required for the following three activities:

1. Insertion/removal of screw

2. Movement of limbs

3. Movement of body

For all the three categories, energy requirement increases with the dynamic pressure of the flow in the vent. A greater dynamic pressure increases the aerodynamic drag, which in turn increases the energy required for moving limbs and body against the flow. A greater drag also requires deeper insertion of ice screw, which in turn requires more energy for inserting and removing the screw. Aerodynamic drag depends on the shape and size of DM. We assumed the prototype design described in Section 7, and estimated the drag by a computational flow dynamic (CDF) simulation.

Figure 25 shows the resulting estimation of required energy for making $1 \mathrm{~m}$ of progress. The basis of the estimation and assumptions are described in detail in the following subsections. 


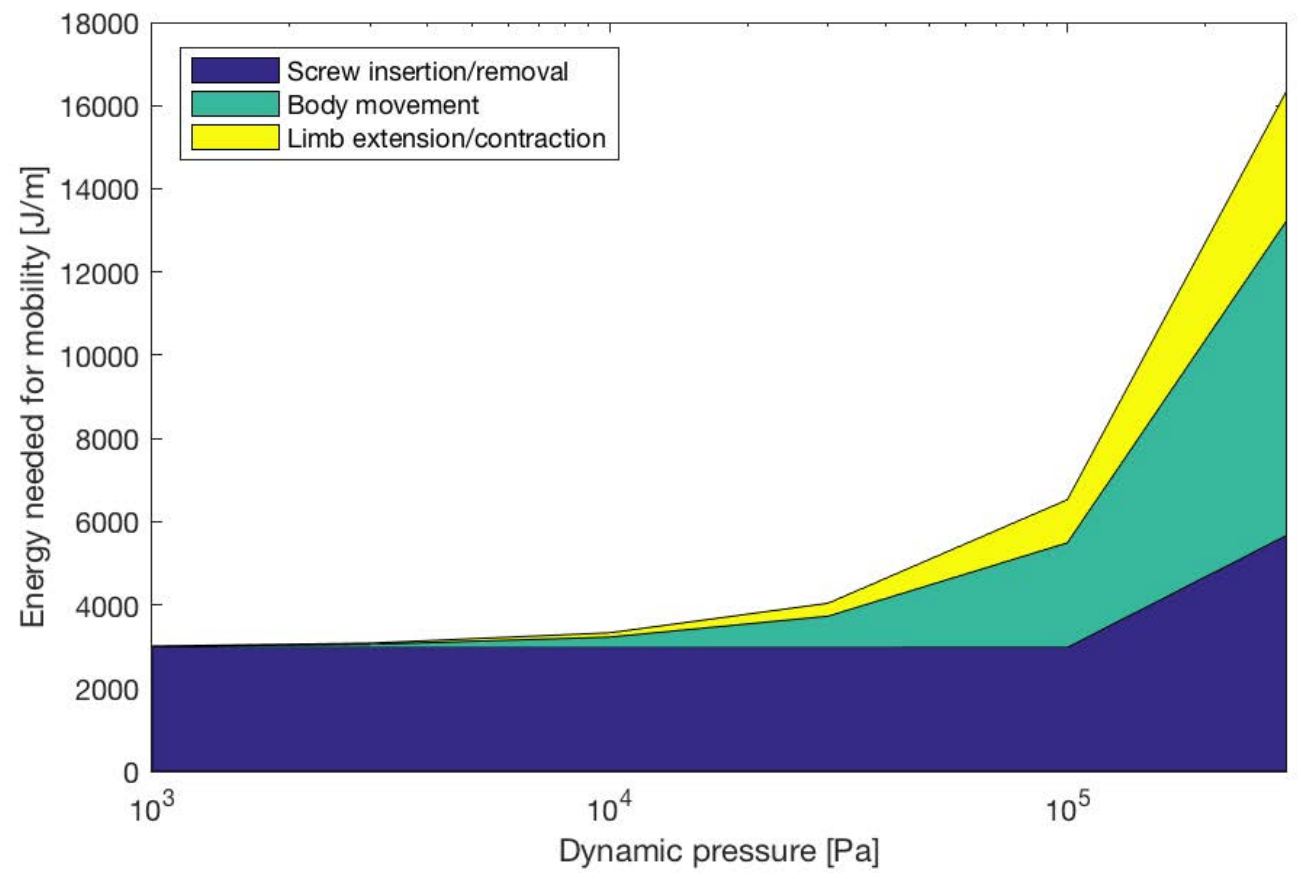

Figure 25 Estimated required energy for a given dynamic pressure of the upward flow in the vent

\subsubsection{CFD Analysis of Aerodynamic Drag}

Since the analysis is supposed to be a first order study, we did not model the actual flow, considering the transonic condition or the different components. Instead, we analyzed the model in a simplified case, imposing a fixed dynamic pressure of $0.5 \mathrm{~Pa}$, and linearly scaled the result. For the analysis, three 3 models where prepared. The first model was set with the limbs open at an angle of approx. $30^{\circ}$. The second model had the limbs parallel and closed. The third model represented the climber without the limbs. The same flow domain has been used for all the three models, then the forces have been extracted for each component.

The model is a k- $\varepsilon$ with an inlet speed of $1 \mathrm{~m} / \mathrm{s}$ and a turbulence of $5 \%$ (standard parameter), the body and the floor are modelled as no slip surfaces, while the other walls are free slip surfaces. The convergence condition is set at $10^{-4}$ for mass and velocity. Since the model is very complex and for the most part doesn't act as an aerodynamic body, no inflation has been added to capture the boundary layer. Instead the mesh has been refined in proximity of the body, with more attention close to any peculiar feature. 


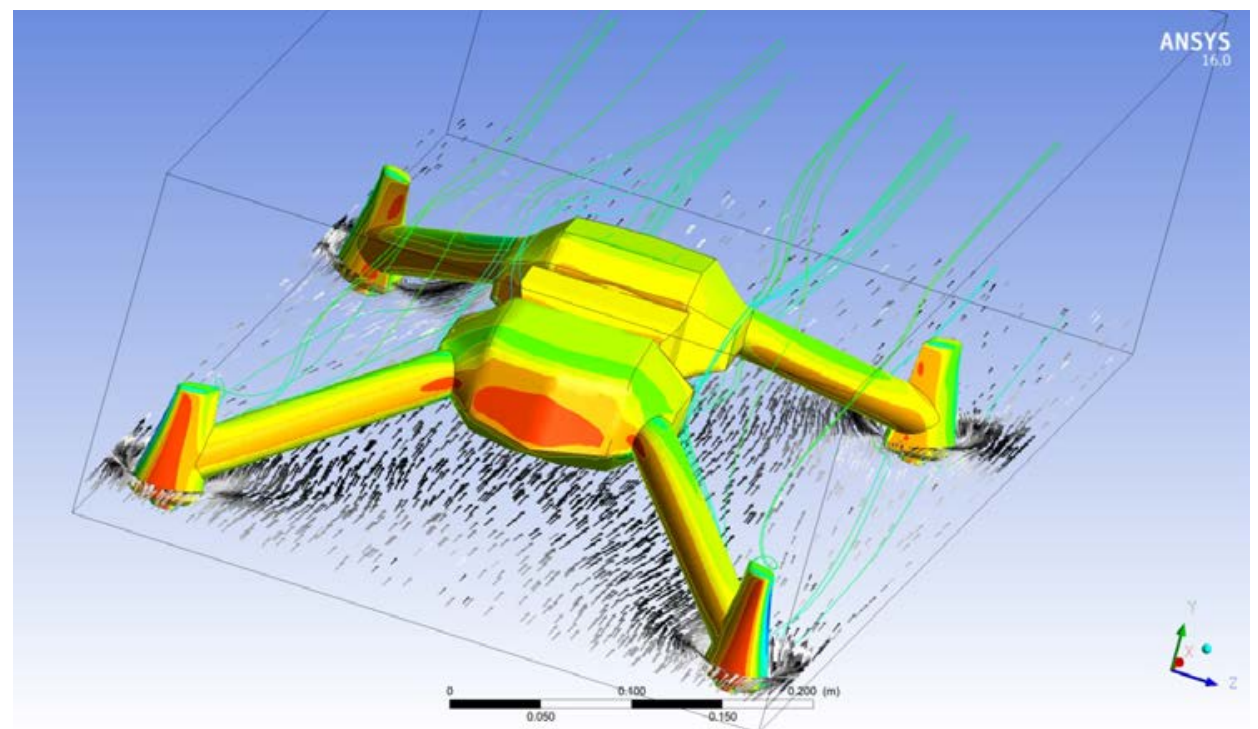

Figure 26 CFD Analysis. Open legs configuration

The results are shown in Table 2 and Figure 27. Note that the closed limb formation results in significantly reduced drag. For the estimation of energy requirement, we assume the closed limb formation. We also assume the drag scales linearly with the dynamic pressure.

\begin{tabular}{lrrrr}
$\begin{array}{l}\text { Aerodynamic drag }(\mathrm{N}) \text { for } \\
\text { dynamic pressure }\end{array}$ & Open limbs & Closed limbs & No limbs \\
\hline Total Body + Legs & 1671 & 744 & \\
Total Body & 466 & 437 & 734
\end{tabular}




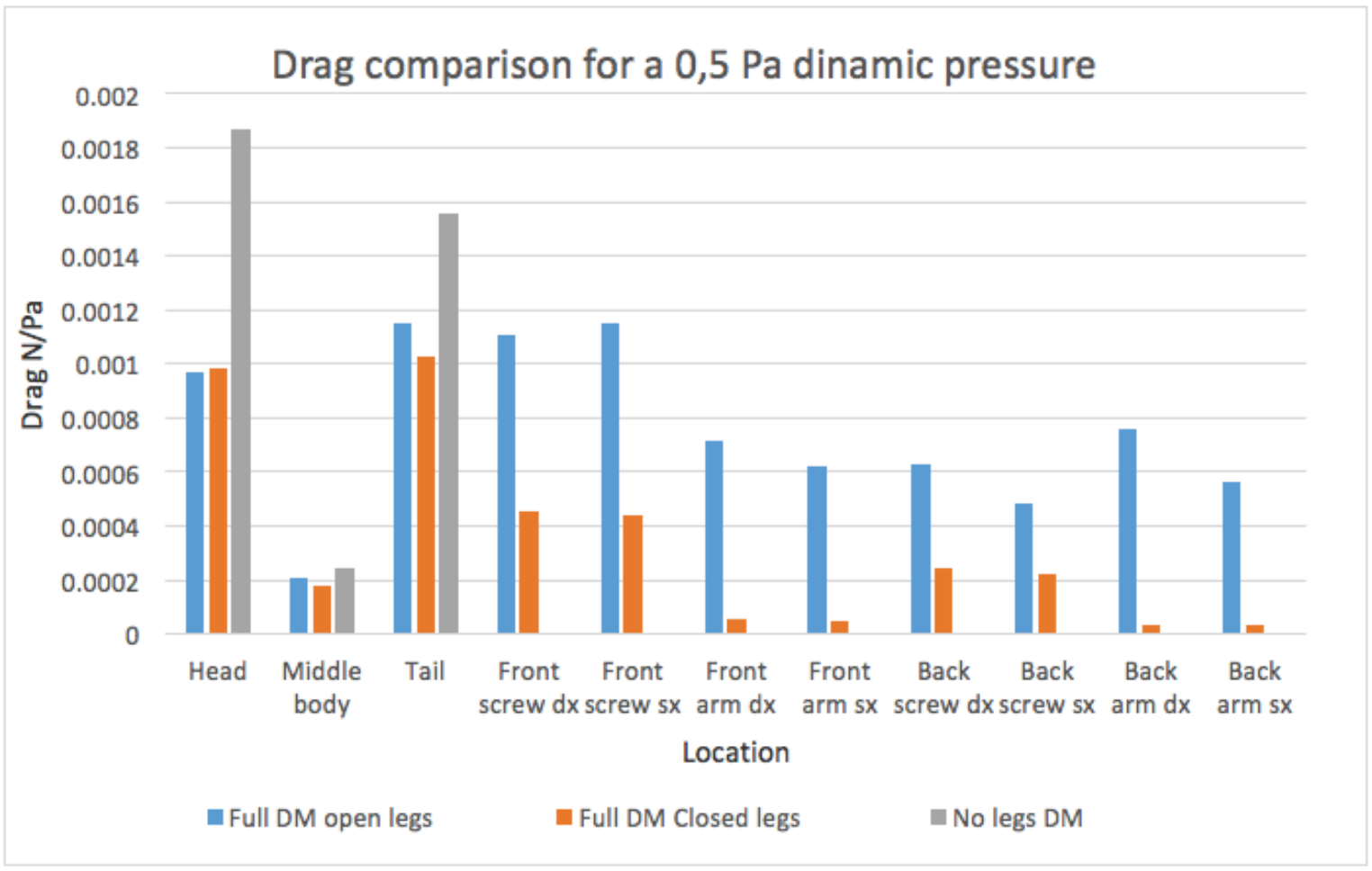

Figure 27 CFD results: drag for individual components

\subsubsection{Energy for body and limb movement}

The energy required for moving the body and limbs is derived from Table 2 with a few assumptions.

- The energy efficiency of motor and gear $\left(\epsilon_{M}\right)$ is 0.37 (from input electric energy to output mechanical work), derived from catalog specs of commercially available miniature, high energy density motor and gear ${ }^{6}$.

- The energy efficiency of the linear joint of the limb $\left(\epsilon_{J}\right)$ is 0.8 .

For the aerodynamic drag force $\left(F_{D}\right)$ from Table 2, the required energy needed to move by $1 \mathrm{~m}$ is given by:

$$
E=\frac{F_{D}}{\epsilon_{M} \epsilon_{J}}
$$

\subsubsection{Energy for screw insertion/removal}

We found very limited literature on the quantitative analysis of required energy and strength of ice screw, probably because ice screw is primarily used not for scientific/engineering

\footnotetext{
${ }^{6}$ We assumed Maxon's DCX $19 \mathrm{~S}$ motor $(12 \mathrm{~V})^{6}$, which has $1.9 \mathrm{~cm}$ diameter, $3.4 \mathrm{~cm}$ length, and >$40^{\circ} \mathrm{C}$ operational temperature. At the nominal operation point, it outputs $11 \mathrm{mNm}$ of torque and rotates at $4490 \mathrm{rpm}$, with $79 \%$ maximum efficiency. As for the energy efficiency of gear, we refer to Maxon's GPX 22 HP 4-stage planetary gear ${ }^{6}$, which has up to 439:1 gear ratio and 55\% energy efficiency.
} 
applications but for mountaineering. Therefore, we performed ice chamber experiments to find first-order estimates.

First, to estimate the required insertion depth of ice screws, we experimentally assessed the maximum shear force an ice screw can tolerate for a give $\mathrm{n}$ insertion length. The experimental setup is shown in Figure 28. We used the same Black Diamond ice screw with $19.5 \mathrm{~mm}$ diameter. The result is shown in Figure 29. The details of the experiments are described in Section 6.2.
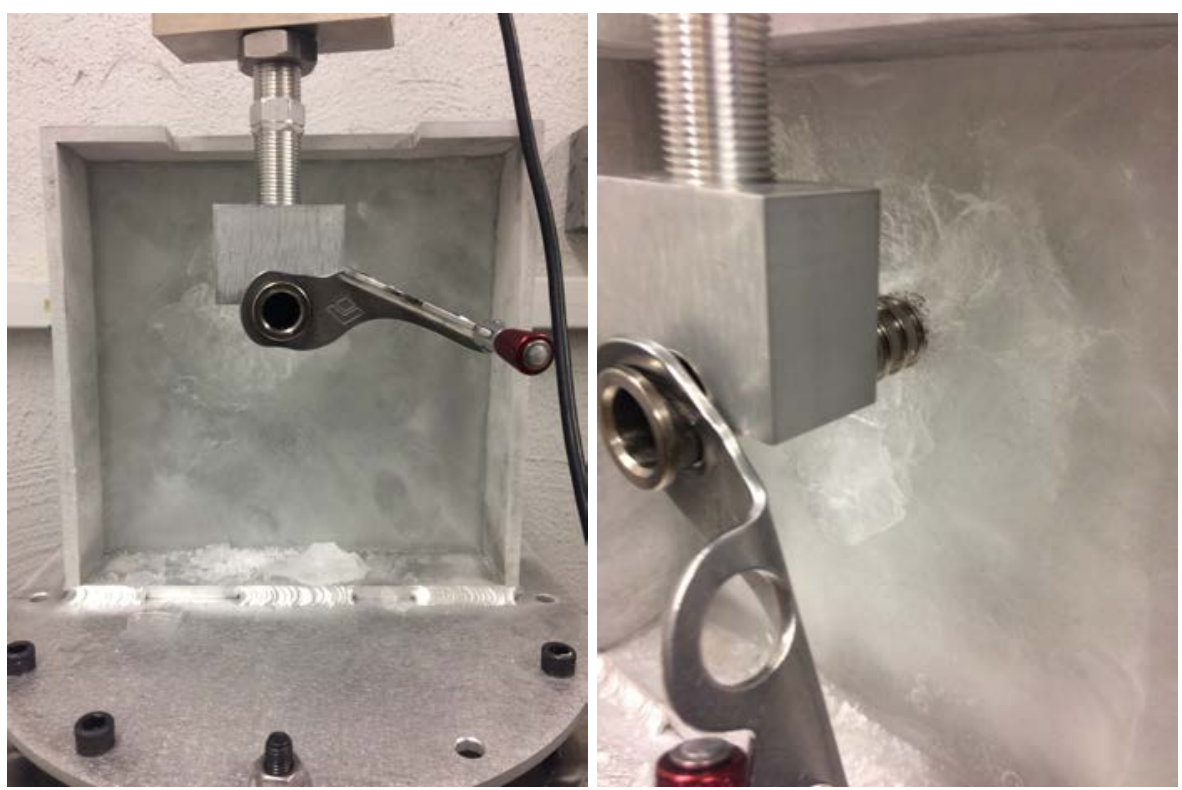

Figure 28 Experimental setup to measure the tolerable sheer force of ice screw. The ice screw is inserted to ice and pushed downwards by a pressing stand.

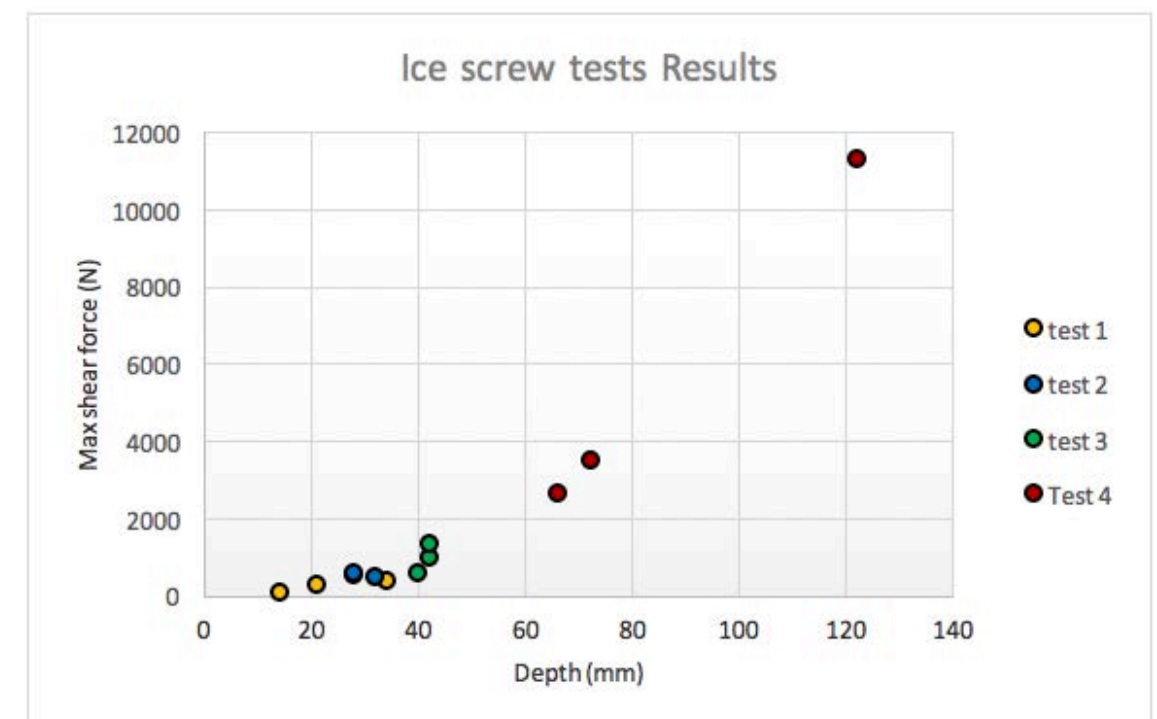

Figure 29 Result of experiments, showing the maximum sheer force for a given insertion depth. 
From the experimental results, we made the following assumptions:

- Insertion depth needs to be at least twice the diameter of screw in order to make an effective anchor

- With the minimum insertion depth, the $19.5 \mathrm{~mm}$ screw can support $900 \mathrm{~N}$ of sheer force

- With a greater insertion depth than the minimum, the maximum sheer force increases linearly at the rate of $125 \mathrm{~N} / \mathrm{mm}$

- For a screw with different diameter (D) and insertion depth (L), the maximum sheer force is proportional to $\mathrm{DL}$

- The energy efficiency of motor and gear is 0.37 (from input electric energy to output mechanical work), derived from catalog specs of commercially available miniature, high energy density motor and gear ${ }^{7}$.

With these assumptions and the CDF results in Table 2, we computed the necessary insertion depth.

Next, we performed experiments (Figure 30) to assess the energy required for inserting and removing ice screw for a given insertion depth. We used a $10 \mathrm{~cm}$ ice screw manufactured by Black Diamond, which is $19.5 \mathrm{~mm}$ in diameter, and a pristine water ice. The maximum torque was 3.6 $\mathrm{Nm}$. It required $\sim 500 \mathrm{~J}$ of energy to insert $8 \mathrm{~cm}$ of the screw and then remove it. The details of the experiment are described in Section 6.1.

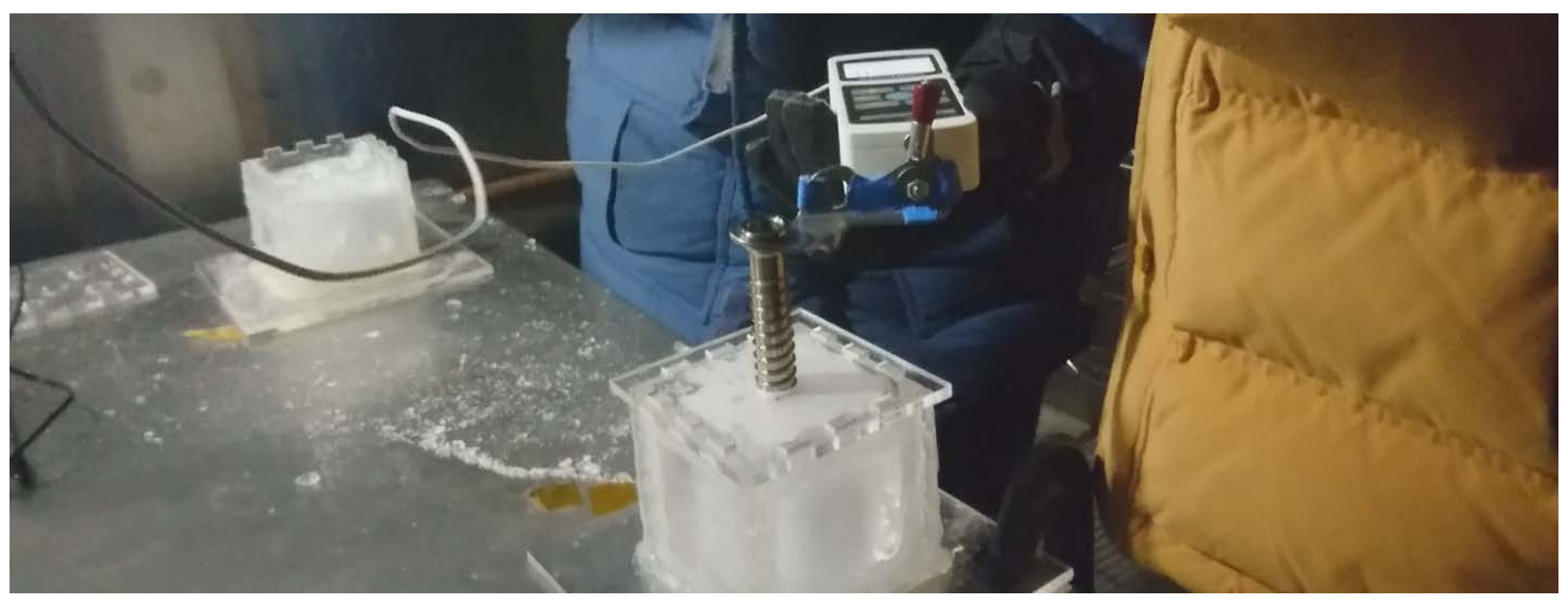

Figure 30 Experiment to measure the energy required to insert and remove an ice screw

\footnotetext{
${ }^{7}$ We assumed Maxon's DCX $19 \mathrm{~S}$ motor $(12 \mathrm{~V})^{7}$, which has $1.9 \mathrm{~cm}$ diameter, $3.4 \mathrm{~cm}$ length, and $>-40^{\circ} \mathrm{C}$ operational temperature. At the nominal operation point, it outputs $11 \mathrm{mNm}$ of torque and rotates at $4490 \mathrm{rpm}$, with $79 \%$ maximum efficiency. As for the energy efficiency of gear, we refer to Maxon's GPX 22 HP 4-stage planetary gear ${ }^{7}$, which has up to 439:1 gear ratio and 55\% energy efficiency.
} 
The ice screw used for DM is smaller than the one used for the experiment. To estimate the energy for different screw diameter $(d)$ and insertion depth $\left(l_{I}\right)$, we conservatively assumed that the energy is proportional to $d l$ (as opposed to the volume).

To complete one locomotion cycle (see Figure 24), DM needs to repeat screw insertion/removal four times. The distance DM travels with one locomotion cycle is equivalent to the stroke length of limbs $\left(l_{S}\right)$ (same as the stroke length). Our energy estimate is given by:

$$
E=\frac{4}{l_{S}} \cdot \frac{d}{19.5 \mathrm{~mm}} \cdot \frac{l_{I}}{100 \mathrm{~mm}} \cdot 500 \mathrm{~J}
$$

\subsubsection{Power Source Trade Study}

Next, we compare options for powering the DM. Below are the five options considered in the trade study.

1. Primary battery on DM; no tether

2. Miniaturized RTG on DM; no tether

3. Primary battery on SM, supplied to DM through tether

4. Solar cell on SM with tether, supplied to DM through tether

5. RTG on SM with tether, supplied to DM through tether

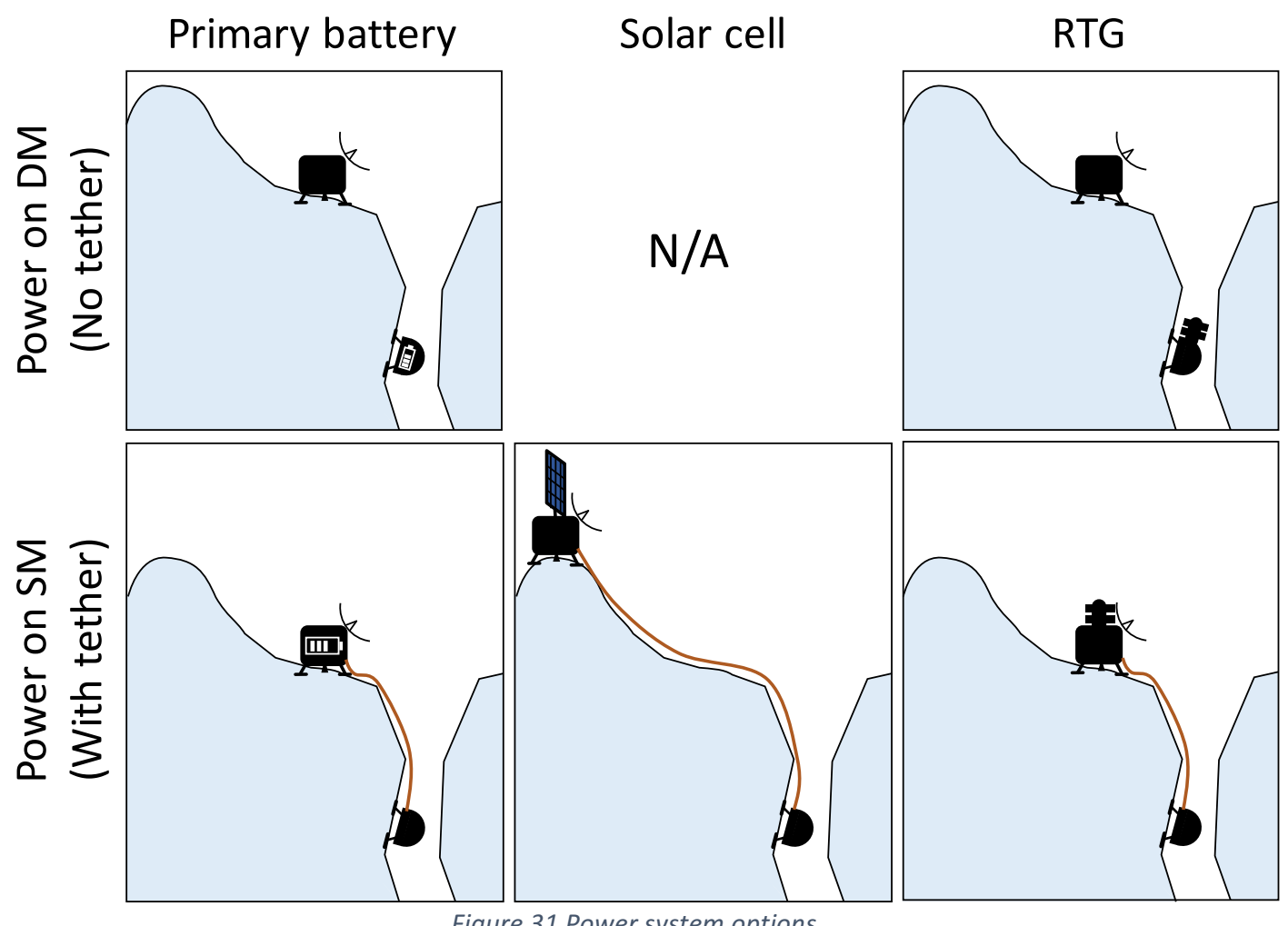

Figure 31 Power system options 
These options are compared by three criteria:

A) The distance DM can travel

B) The speed DM can travel

C) Maximum number of DMs

D) Major risks involved in the energy source

The major assumptions of the analysis include:

- For "power on DM" options, DM has five additional middle sections to accommodate battery/RTG.

- Each additional module is hemispheric with a $5 \mathrm{~cm}$ radius

- We did not take the increased aerodynamic drag due to additional sections in to account. Therefore, the estimates for "power on DM" options must be view as optimistic.

- For the "no tether" options, SM's the minimal power requirement is the same as Europa Lander - $1100 \mathrm{Wh} /$ day or $45 \mathrm{~W}$, including minimal communications for background engineering and survival heater energy (Hand, et al., 2017).

- $\quad$ Each DM uses up to $10 \mathrm{~W}$ of power for mobility

The result of the analysis is summarized in Table 3. The basis of estimations and assumptions for each configuration are described in the following subsections.

Table 3 Comparison of power systems. The distance of "power on DM" configurations is the distance that can be traveled by each $D M$, while that of "primary battery on SM" is the total distance traveled by all DMs combined. The distance of "power on DM"RTG is bounded not by available energy but by mission duration due to its slow speed. Max number of DMs of "power on DM" configurations is bounded by the payload mass of SM, while that of "power on SM" configurations is bounded by power, hence it represents the number of DMs that can be operated simultaneously. (SM can bring more DMs.)

\begin{tabular}{|c|c|c|c|c|c|}
\hline Configuration & $\begin{array}{l}\text { Energy } \\
\text { source }\end{array}$ & $\begin{array}{l}\text { Distance } \\
\text { (m) }\end{array}$ & $\begin{array}{l}\text { Speed } \\
(\mathrm{m} / \mathrm{hr})\end{array}$ & $\begin{array}{l}\text { Max } \\
\# \text { of } \\
\text { DMs }\end{array}$ & Major risk \\
\hline \multirow[t]{2}{*}{$\begin{array}{l}\text { Power on DM } \\
\text { (No tether) }\end{array}$} & $\begin{array}{l}\text { Primary } \\
\text { battery }\end{array}$ & 350 & 5.5 & 10 & Unreliable comm. \\
\hline & RTG & $\begin{array}{l}1300 \text { (per } \\
\text { year) }\end{array}$ & 0.15 & 10 & Unreliable comm. \\
\hline \multirow[t]{3}{*}{$\begin{array}{l}\text { Power on SM } \\
\text { (With tether) }\end{array}$} & $\begin{array}{l}\text { Primary } \\
\text { battery }\end{array}$ & $\begin{array}{l}2600 \text { (all } \\
\text { DMs } \\
\text { combined) }\end{array}$ & 5.5 & $1-3$ & \\
\hline & Solar cell & & 5.5 & $1-2$ & $\begin{array}{l}\text { Needs at least } 20 \mathrm{~m}^{2} \text { solar cell; Landing site } \\
\text { constrained by sunshine }\end{array}$ \\
\hline & RTG & & 5.5 & $>10$ & \\
\hline
\end{tabular}


From the analysis, we conclude that "RTG on SM" is the most viable option, while "RTG on DM", "primary battery on SM", and "solar cell on SM" are challenging but possible. Each configuration is discussed in further detail in the following subsections.

\subsubsection{Primary Battery on DM}

We assumed the energy density of non-rechargeable lithium battery (1200 Wh/L). We also assumed that $80 \%$ of the five battery sections of DM can be used for batteries, and $50 \%$ of the energy is used for mobility.

Even with these optimistic assumptions, each DM can only travel $350 \mathrm{~m}$. Assuming $10 \mathrm{~W}$ power for mobility, the lifetime of DM is just 60 hours. Given $100 \mathrm{~m}$ landing accuracy of SM, significant portion of the range is consumed by moving from SM to vent. With this configuration, only the shallow portion of vents can be explored with very limited time. This drawback can be partially mitigated by bringing a large number of DMs. However, assuming the payload mass of Europa Lander ( $65 \mathrm{~kg})$ and the increased mass of DM due to battery ( $6 \mathrm{~kg})$, only $10 \mathrm{DMs}$ can be accommodated. Therefore, we conclude that this configuration is not a viable option.

\subsubsection{RTG on DM}

We assumed miniaturized RTGs with the same power density as the multi-mission RTG (MMRTG) fill $80 \%$ of the volume of the five additional sections. An MMRTG generates $\sim 125 \mathrm{~W}$ at the beginning of mission, which falls to $\sim 110 \mathrm{~W}$ at the end of the 14-year designed lifetime ${ }^{8}$. Its envelope volume including the radiation panels is $\sim 0.21 \mathrm{~m}^{3}$. With these assumptions, RTG on DM can produce $0.5 \mathrm{~W}$ of power, $50 \%$ of which is assumed to be used for mobility. This results in the speed of $0.15 \mathrm{~m} / \mathrm{hr}$.

The extremely slow speed is a major challenge of this configuration. If operated continuously, DM can cover $\sim 1300 \mathrm{~m}$ per year, but in practice, even a highly-automated DM would need to pause for mapping and scientific observations, hence the distance could be significantly shorter. Still, if a long mission duration is permitted, this is a possible option.

A unique challenge is that the radiation panels of RTG would create strong aerodynamic forces. A specialized design of RTG with aerodynamic shape would be required.

\subsubsection{Primary battery on SM}

We use the same specifications as the Europa Lander concept: $45 \mathrm{kWh}$ battery capacity and 20day lifetime on surface. After subtracting $45 \mathrm{~W}$ (=22 kWh for 20 days) minimum energy requirement, $23 \mathrm{kWh}$ is available for DM. With this energy, DM can travel $2600 \mathrm{~m}$. If there are multiple DMs, this is the distance traveled by all the DM collectively. With the limited range and mission life time, returning samples to SM would not make sense, and there would be no time for scouting. Hence, all the DMs would be for in-situ science.

${ }^{8}$ https://ntrs.nasa.gov/archive/nasa/casi.ntrs.nasa.gov/20080003866.pdf 
This configuration is not impossible but not plausible.

\subsubsection{Solar cell on SM}

As the vents on Enceladus are in the south polar region, SM may have continuous sunlight in the southern summer by carefully choosing the landing site. On the other hand, a solar-driven SM cannot operate during the southern winter. Therefore, the mission life time is upper bounded by the half orbital period of Saturn, which is 14.7 years. (Enceladus's axis tilt and orbital inclination are nearly zero, and Saturn has $26.7^{\circ}$ axial tilt. Therefore, Enceladus experiences the same seasonal changes as Saturn.)

The solar radiation intensity at Saturn is $15 \mathrm{Wm}^{-2}$. Assuming $20 \%$ efficiency, at least $15 \mathrm{~m}^{2}$ area of solar cell is necessary for SM's survival. In order to operate DMs, at least $20 \mathrm{~m}^{2}$ would be necessary. The solar cells must be gimbaled to chase the Sun. Due to the low elevation angle of Sun in the south polar region, the solar cells must be deployed vertically, which is challenging but possible under the weak gravity of Enceladus (0.01g).

In this configuration, DM needs to travel a greater distance to the vent, as SM's landing site must be determined based primality on the lighting condition instead of the proximity to vents. For example, if SM lands on the ridge of one the "Tiger Stripes," DM would need to travel a few kilometers (Porco, et al., 2006) to reach the mouth of a vent.

\subsubsection{RTG on SM}

We assume SM has two MMRTGs, which produces 110W each. The mass of two MMRTGs (90 $\mathrm{kg}$ ) is similar to the mass of the $45-\mathrm{kWh}$ primary battery of Europa Lander ( $95 \mathrm{~kg}$ including packaging). The range of DM is limited not by energy but by tether. The abundance of power allows to operate more than ten DMs at their top speed $(5.5 \mathrm{~m} / \mathrm{hr})$. There are no limitations on landing site and mission duration. Therefore, we conclude that this configuration is the most viable one for EVE.

\subsection{Tether System}

There are two configurations for tether:

1. "Spool on SM" option: SM stores tether and DM pulls it out

2. "Spool on DM" option: DM stores and deploys tether 


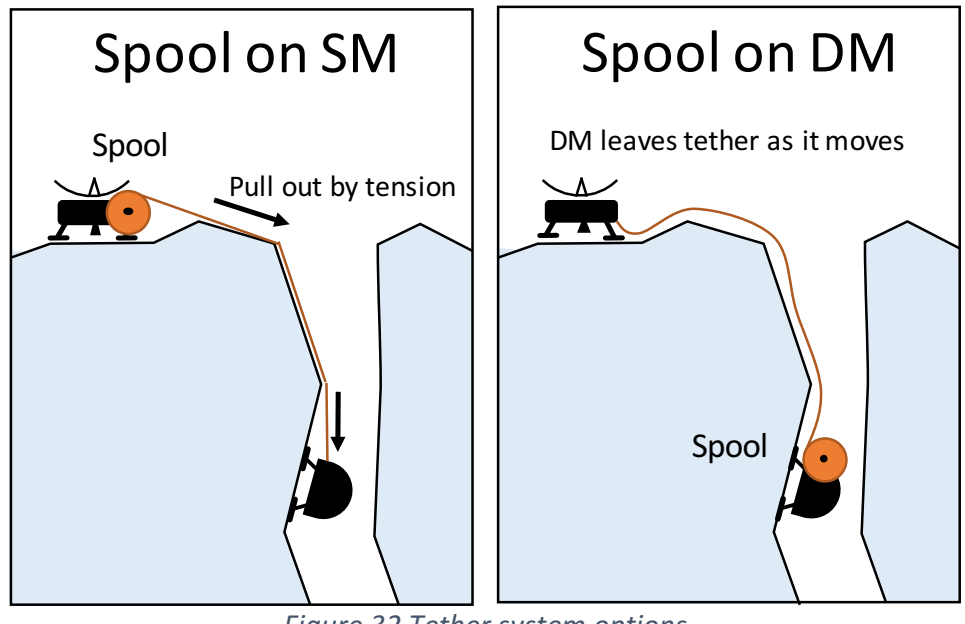

Figure 32 Tether system options

The "spool on SM" option would have a major technical difficulty in tether deployment, as DM must provide the tension to pull it out. As a result, the tether must have mechanical strength to support the tension. Furthermore, the tether must move with DM. Tether's abrasion and entanglement could disable DM. Therefore, this option is realistic only for a short distance.

The "spool on DM" does not have difficulty in deployment because no tension is required for deployment, and the tether does not have to move once deployed. A disadvantage is that the tether length is limited by DM's storage capacity, which in turn limits the maximum depth that the mission can explore.

We made a first-order estimate of the required storage volume for the "spool on DM" option. We assume a $2000 \mathrm{~m}$ long 38 AWG (American Wire Gauge) insulated copper magnet wire (Figure $33^{9}$ ), which has $0.101 \mathrm{~mm}$ diameter. Three copper wires are assumed to be used for GND, power, and data lines. The total volume of the wires is $4.8 \times 10^{-5} \mathrm{~m}^{3}$ or $48 \mathrm{~cm}^{3}$, which is about $20 \%$ of the volume of DM's rear section. While a longer tether could be accommodated, the rear section also needs to accommodate the mechanical system for actuating two limbs (details in Section 7). The $20 \%$ allocation for tether in the rear section would be a reasonable option. Alternatively, $\sim 10 \mathrm{~km}$ tether is possible by adding a dedicated section for tether, at the cost of increased aerodynamic drag. The electric resistance of the $200 \mathrm{~m}$ cable is $\sim 600 \mathrm{ohm}$, which results in $45 \%$ loss of energy when

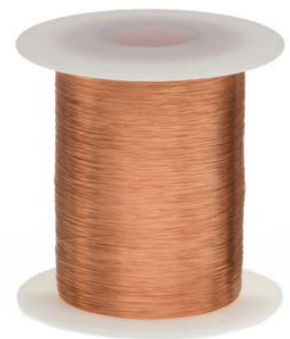
transmitting $10 \mathrm{~W}$ at $100 \mathrm{~V}$.

\footnotetext{
9 Data and image taken from Remington Industries's webpage: http://www.remingtonindustries.com/magnetwire/magnet-wire-38-awg-2-oz-2494-length/?utm medium=googleshopping\&utm source=bc
} 
Based on this analysis, we conclude that "spool on DM" is the most realistic configuration. The possible length of tether is $\mathbf{2} \mathbf{~ k m}$ if the spool is accommodated in the rear section, or $\mathbf{1 0} \mathbf{k m}$ if a dedicated tether section is added.

\subsection{Autonomy System}

Unlike existing Mars rovers, which are mostly controlled manually, EVE's DMs should be highly automated for several reasons.

i) Unavailability of orbital reconnaissance: In strategic (i.e., long-distance) planning of Mars rovers, rover planners are greatly assisted by the high resolution $(25 \mathrm{~cm})$ orbital images provided by Mars Reconnaissance Orbiter's HiRISE camera, as well as the digital elevation model (DEM) created from stereo HiRISE images. The rover planners have a bird's-eye view, in which they can identify topology, terrain type, and obstacles beyond the rover's line of sight. EVE will not have this luxury.

ii) Poor viewshed: In tactical (i.e., day-to-day) planning of Mars rovers, the drive distance per operation cycle (Sol) by manual planning is limited by viewshed (geographical area that is visible from a location), which is typically $50 \mathrm{~m}$. In the vent, it would be tens of $\mathrm{cm}$. The operation cycle of EVE would be the same as Enceladus's 1.37-day orbital period due to the co-orbiting CRO. Limited to tens of $\mathrm{cm}$ per operation cycle, it would take decades to move just one kilometer.

iii) Multiple DMs: Past space missions have operated only one or two spacecraft. In EVE, up to tens of DMs must be operated simultaneously. Operating multiple DMs would not only increase the labor and cost if manually operated, but would also limit the amount of data to be downlinked from each DM. On-board decision making would remove this bottleneck.

iv) Dynamic environment: When problems occur, Mars rovers can safely stop anytime and wait for ground control because the environment of Mars is largely static. In contrast, Enceladus's vent-conduit system is dynamic. DMs will have to take immediate actions upon the detection of anomalies, rather than stopping and waiting for ground response.

Figure 34 presents an autonomy capability roadmap, which shows required mission-level, system-level, function-level, and algorithm-level autonomy capabilities with dependencies among them. Capabilities in one column are linked to enabling/supporting capabilities in the 
next column to the right. Narratives for each of the system-level capabilities are provided below.

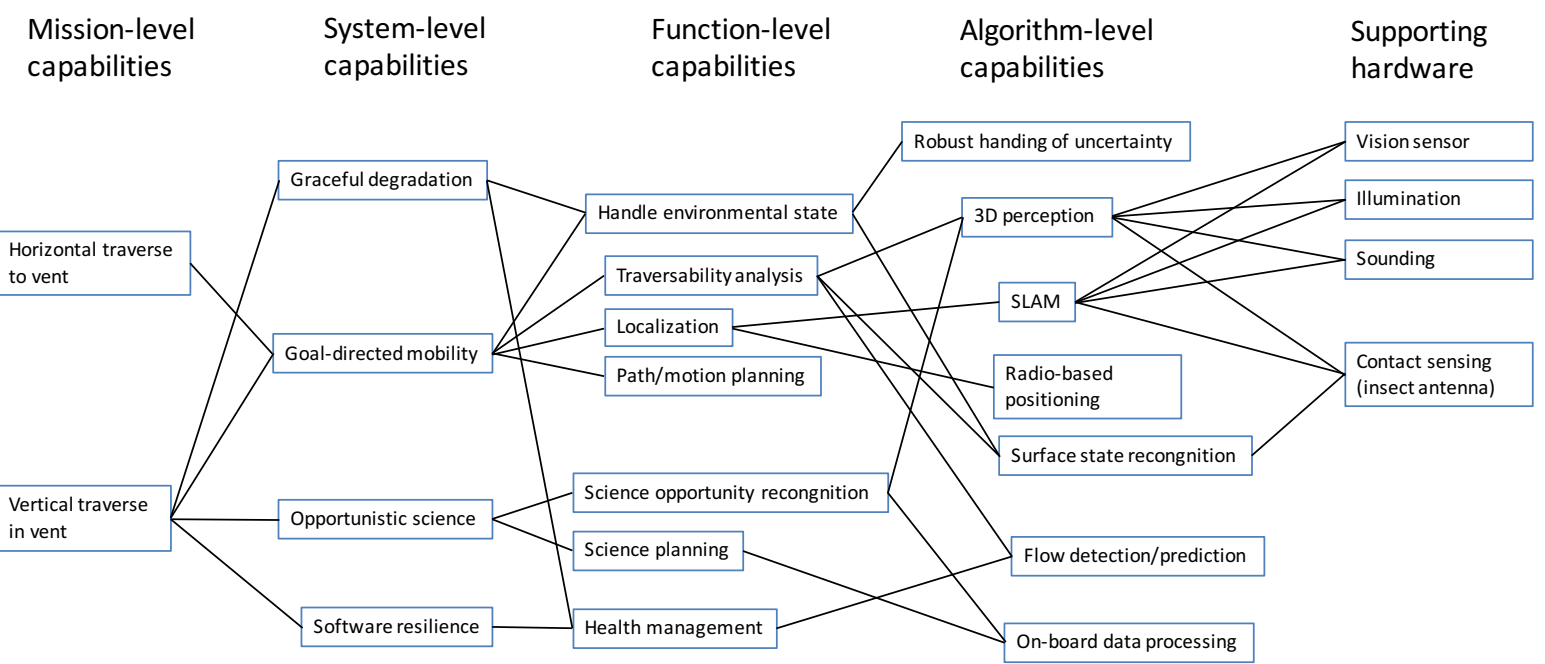

Figure 34 Autonomy Capability Roadmap for EVE

\subsubsection{Graceful degradation}

Mechanical complexity of DM is a double-edged sword. It would undermine the success of the mission if DM's software is designed in a way that any single mechanical failure results in the shut-down of the DM. However, it benefits the mission if the software is designed to exploit the flexibility of the mechanical system and reconfigure it upon mechanical failures. For example, a human soldier is not immediately incapacitated by an injury to one of his limbs. Instead, he would quickly adapt to the change in his physical system and stay active by using his remaining limbs. Likewise, if one of the ice screws of a DM is failed or one of the joints is locked, the DM's software should adapt to the change in its hardware system by changing the gait and continuing the mission at a degraded rate.

\subsubsection{Goal-directed mobility}

Because the global map of the vent-conduit system is not available, the ground operator cannot specify physical locations for a DM to go beyond the regions already explored by other DMs or its own very limited line of sight. Therefore, DM should take a higher-level goal, such as "go as deep as possible" and "maximize the chance to reach the liquid interface." It has to identify route options to achieve the goals, estimate the traversability and risk of the route options, update the map as it gathers new information, share the information with other DMs, and back up if it encounters difficulty in traversal or judges that the route ahead is too risky.

\subsubsection{Opportunistic science}

Mars rover Curiosity takes more images than it can transmit to Earth due to the bandwidth limitation. Due to EVE's more limited communication bandwidth and the multiple DMs, only a fraction of collected data would be able to return to Earth. However, we do not want DMs to unintelligently pass by evidence of life without noticing it. An onboard capability to autonomously 
detect scientifically interesting scenes, collect relevant data, and select the data to transmit to Earth within the bandwidth limit, is highly desired.

Opportunistic science is not merely an alternative to conventional ground-in-the-loop science but an enhancement of it. As Dr. Penny Boston stated in her keynote speech at the 2016 NIAC Symposium, human scientists repeat various mental activities at a high frequency, e.g., scan at regional scale and focus on general site types, scan at walking scale and focus on individual features or site, and perform in situ analysis by focusing on restricted features. The daily iterative process of MSL, for example, is "not the optimal way to do science in a new environment because it is missing a lot of components that the human naturally brings into it (Boston, 2016)." While realizing such a capability is technically challenging, it would bring a significant benefit for future robotic explorations like EVE.

\subsubsection{Software resilience}

A current challenge in software engineering is the development of a software system that can detect when one of its software components or subsystems is exhibiting anomalous behavior due to an uncaught defect in the design or implementation of the code, and mitigate/accommodate this anomalous behavior and still meet its execution objectives. Such capabilities are sometimes referred to as "self-healing" software, but are more generally captured under the banner of Software Resilience. This capability to appropriately manage the state of the software system can be considered to be a counterpart to the "graceful degradation" capability which manages the state of the spacecraft hardware system.

\subsection{Perception System}

DM's perception system has dual purposes. It is an essential component of the autonomy system (Figure 34) and also provides science data. Its main focus is to create 3D maps of the interior of the vent-conduit system. Below we compare several candidate systems and compare their advantages and disadvantages.

\subsubsection{Overview}

Since orbiter reconnaissance of the interior of vent is impossible, DMs must map the vent and localize itself. This section discusses the applicability of current sensing technology to the exploration of crevasses in the ice crust of ocean worlds. We evaluate the applicability of stereo, structured light, time-of-flight cameras, and Lidars. We consider five criteria: power consumption, volume, range and accuracy. We discuss acceptable ranges for each criterion. Our study suggests the superiority of assisted stereo in the context of an icy-world mission.

We studied the applicability of current depth sensing technology to icy crevasses. We assumed that depth sensing exclusively supports the two following tasks: 3D mapping for path planning/obstacle avoidance, and visual odometry (VO). We limited our study to sensors that fit inside a $1 \mathrm{dm}^{3}$ robot. We also assumed that the robot remains stationary while imaging. The objective of this study is to list existing applicable sensors, and rank the sensors according to power consumption, size, range, accuracy, and accommodation to low temperatures. 
The depth-sensing technologies that we are considering are: stereo vision, structured light, assisted stereo, time-of-flight camera, spinning Lidar, and radar. In the following, we introduce each technology and present qualitative results illustrating their application to icy surfaces. We then rank each sensor according to the five criteria defined above.

\subsubsection{Candidate Sensing Technologies}

\subsubsection{Stereo}

A stereo camera computes depth by stereopsis (Figure 35). Stereo exhibits a compelling sensing range, low volume and low power consumption. Its accuracy gracefully degrades with range in naturally-lit environments. In a dark environment, a floodlight is required, and range is dictated by the luminous intensity of the light and shutter speed. To enable stereopsis, a stereo sensor needs to find matching patches of pixels across left and right images. As a result, stereo only works for textured surfaces that allow us to match discriminative blocks of pixels. It fails on smooth uniform surfaces such as clean ice.

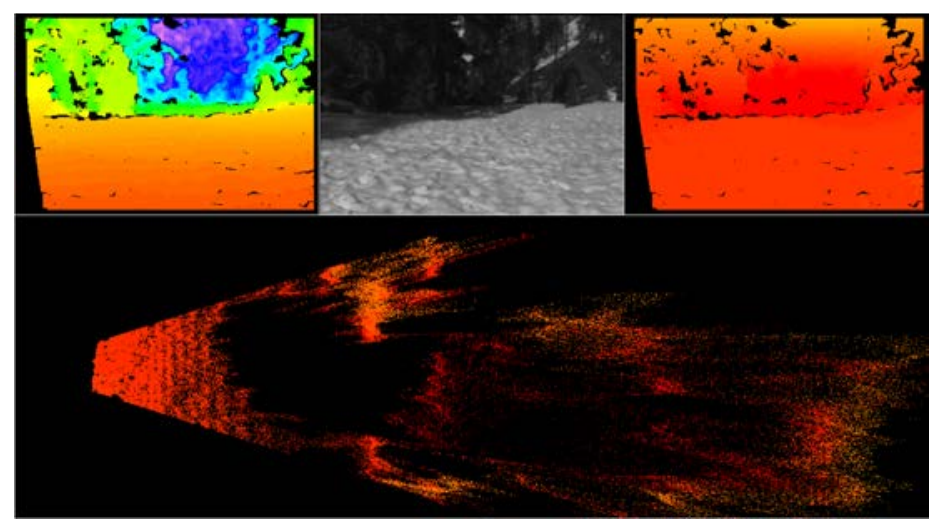

Figure 35: Stereo on snow. Sensor: stereo camera. Material: patchy natural snow. Top-left: stereo-based range image. Top-right: stereo-based elevation map. This figure illustrates that stereo allows us to compute the depth of well-lit textured surfaces. Credit: L. Matthies.

For illustration, we provide this link to a low-power camera sensor:

http://www.himax.com.tw/products/cmos-image-sensor/image-sensor-soc/hm5065/

\subsubsection{Structured Light}

A structured-light sensor projects a light pattern and captures its return with a camera (Figure 36). The deformation of the pattern captured by the camera maps to depth. Range is dictated by the luminous intensity of the projector. By contrast to stereo, structured light cannot leverage natural light to compute depth beyond the reach of its light projector. However, structured light allows us to estimate depth on untextured surfaces. It provides optimal results on Lambertian surfaces. Performances degrade with increased specularity. 

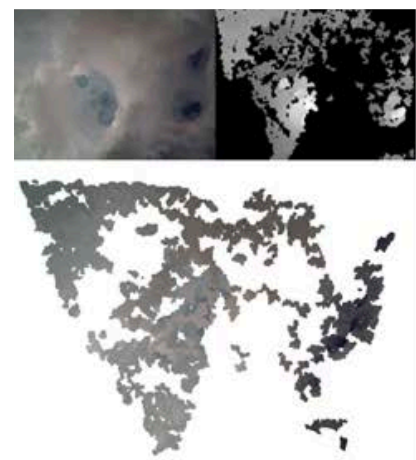

depth: $70 \mathrm{~cm}$
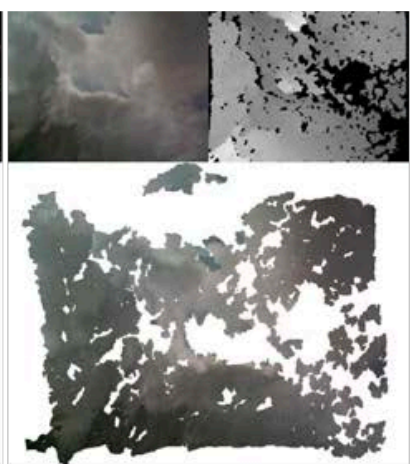

depth: $90 \mathrm{~cm}$
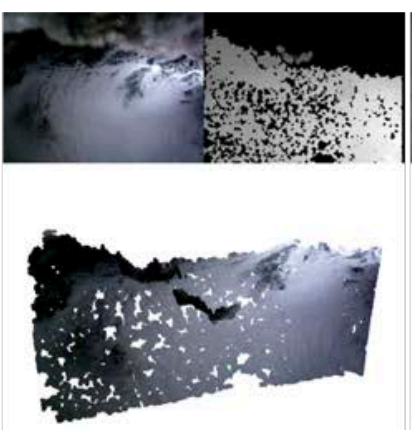

depth: $2 m$
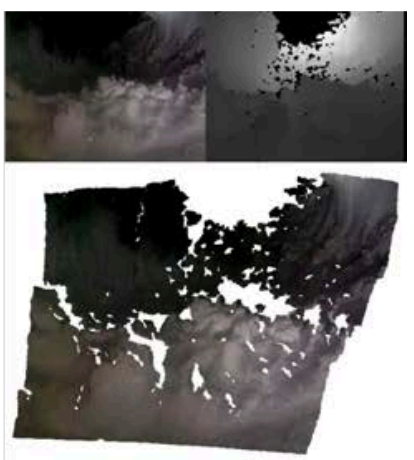

depth: $5 \mathrm{~m}$

Figure 36: Structured light on snow and ice. Sensor: PrimeSense infrared structured light sensor. Material: natural ice and snow at Erebus, Antarctica. Captured by A. Curtis. For each subfigure, the top-left image shows color, the top-right image shows depth, and the bottom image shows the resulting colored point cloud. This figure shows the applicability of structured light for matte surfaces. The sensor has a minimum range of $50 \mathrm{~cm}$. The first subfigure shows this limitation. In the second subfigure, the depth of clear ice cannot be computed.

For illustration, we provide this link to a structured-light sensor:

http://www.i3du.gr/pdf/primesense.pdf

\subsubsection{Assisted Stereo}

Assisted stereo combines the principles of stereo and structured light (Figure 37). It requires more space than stereo or structured light, but it works on untextured surfaces, and it provides depth beyond the reach of the robot light if natural light is present.
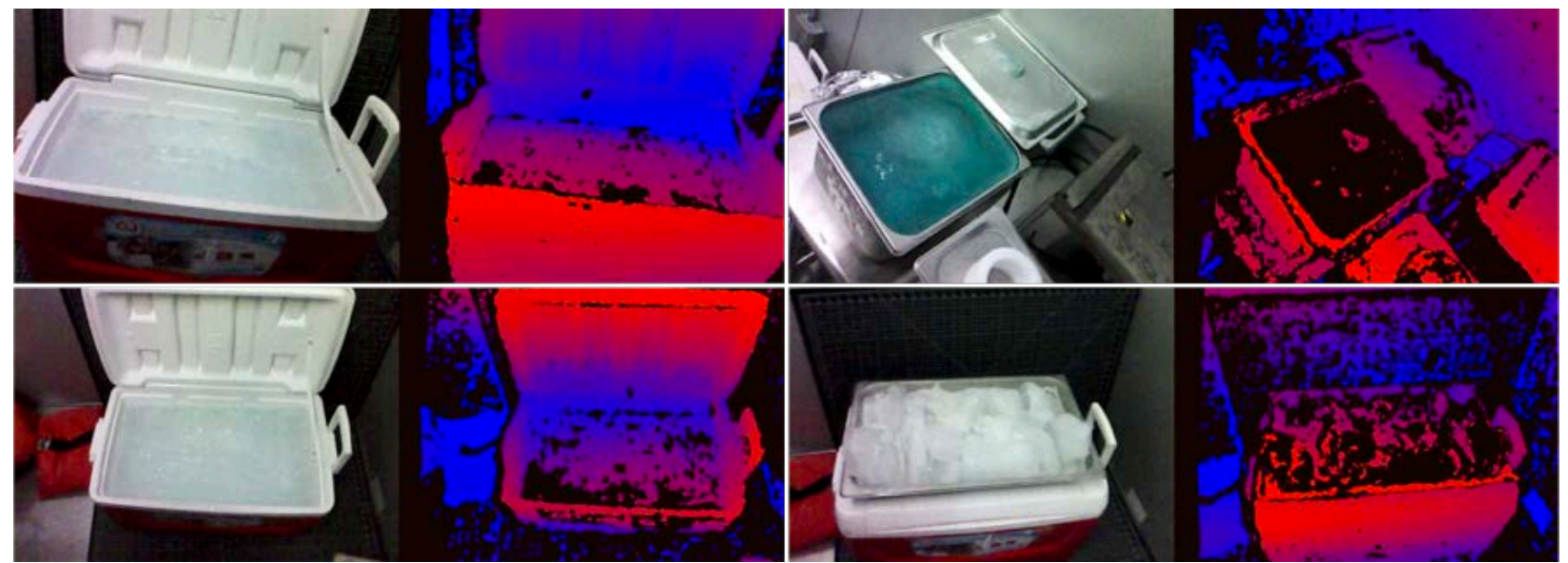

Figure 37: Assisted stereo on ice. Sensor: Intel infrared structured-light sensor. Material: artificial ice at JPL.

The top-right image shows that the sensor does not work on translucent ice. The other images show

convincing results for opaque ice.

For illustration, we provide this link to an assisted-stereo sensor:

https://software.intel.com/en-us/realsense/r200camera

\subsubsection{Time-of-flight Camera}

Time-of-flight cameras measure the return time of a light pulse with a 2D array of receptors, providing scene-wide depth measurements at 25+ frames per second. Current time-of-flight 
cameras provide more reliable measurements than the systems listed above, at the cost of more power, more space, and less range.

For illustration, we provide this link to a ToF camera sensor:

http://downloads.mesa-imaging.ch/dlm.php?fname=pdf/SR4000_Data_Sheet_rev1.5.pdf

\subsubsection{Spinning Lidar}

Lidar sensors measure the return time of a narrow light pulse (Figure 38). To generate a range image, the scene is scanned by panning and tilting the Lidar. Lidars are generally more accurate than camera-based systems, at the cost of more power and more space. Their reliance on mechanical scan can potentially make them sensitive to temperature.

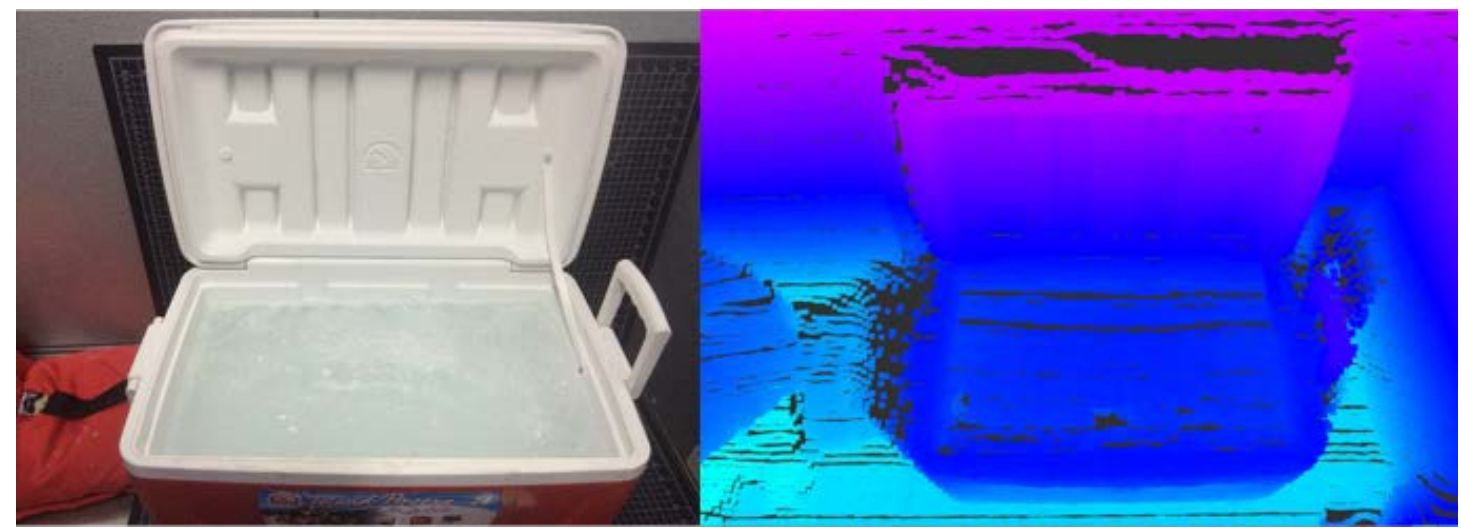

Figure 38: Spinning Lidar on ice. Sensor: Hokuyo infrared spinning Lidar. Material: artificial ice at JPL. This figure shows the applicability of a spinning Lidar to range sensing on opaque ice.

For illustration, we provide this link to a Lidar sensor:

https://www.hokuyo-aut.jp/search/single.php?serial=170

\subsubsection{Radar}

We included Radar in the discussion for completeness. The accuracy of current Radar systems is an order of magnitude poorer than that of other sensors considered in this study.

\subsubsection{Discussion}

(Assisted) stereo, structured light, ToF cameras and Lidar are typically operated either in near-IR or visible. Operating at other wavelengths is in principle feasible, with no obvious disparity in the effort required for enabling a new operating wavelength.

Figure 39 summarizes our discussion. We note that

- For the three camera-based solutions, FPGA-based stereo processing would use 1 to 30 watts for $0.2 \mathrm{~s}$.

- Sensing range and accuracy assume that there is no natural lighting; artificial lighting is included in all power estimates.

- The relevance of the criteria considered in this study will vary depending on the task. 
- We built this table based on our experience with commercial sensing devices and lighting equipment. It is possible that one could build - for instance - a flash (non-scanning) Lidar device specifically for this application, that would perform better than assisted stereo on all counts. ToF cameras/Lidars are currently evolving at a fast pace, driven partly by the emerging self-driving car industry. Solid-state Lidars could have an important impact on this problem.

In summary, this exercise indicates that assisted stereo and structured light are the two technologies that best fit the constraints of icy-world crevasse exploration at this moment. This problem will need to be re-evaluated as solid-state Lidar continues to progress.

\begin{tabular}{|c|c|c|c|c|c|c|c|c|c|c|}
\hline & \multicolumn{2}{|c|}{$\begin{array}{l}\text { Power } \\
\text { Consumption }\end{array}$} & \multicolumn{2}{|c|}{$\begin{array}{l}\text { Device } \\
\text { Volume }\end{array}$} & \multicolumn{2}{|c|}{$\begin{array}{l}\text { Sensing } \\
\text { Range }\end{array}$} & \multicolumn{2}{|c|}{$\begin{array}{l}\text { Sensing } \\
\text { Accuracy }\end{array}$} & \multicolumn{2}{|c|}{$\begin{array}{l}\text { Temperature } \\
\text { Resistance }\end{array}$} \\
\hline $\begin{array}{l}\text { Stereo } \\
\text { Vision }\end{array}$ & 3 & $\begin{array}{l}5 \mathrm{~W} \\
\text { exposure: .1s- } \\
\text { inf }\end{array}$ & 4 & $2 \times 1 \mathrm{~cm}^{3}$ & 4 & $\begin{array}{l}0.1 \mathrm{~m} \text {-inf } \\
\text { (further } \\
\text { with } \\
\text { longer } \\
\text { exposure) }\end{array}$ & 2 & $\begin{array}{l}\text { Fails if no } \\
\text { texture }\end{array}$ & 3 & \\
\hline $\begin{array}{l}\text { Structured } \\
\text { Light }\end{array}$ & 3 & $\begin{array}{l}7.5 \mathrm{~W} \\
\text { exposure: .1s- } \\
\text { inf }\end{array}$ & 4 & $\begin{array}{l}2 \times 1 \\
\mathrm{~cm}^{3}\end{array}$ & 4 & $\begin{array}{l}0.1 \mathrm{~m} \text {-inf } \\
\text { (further } \\
\text { with } \\
\text { longer } \\
\text { exposure) }\end{array}$ & 3 & & 3 & \\
\hline $\begin{array}{l}\text { Assisted } \\
\text { Stereo }\end{array}$ & 3 & $\begin{array}{l}7.5 \mathrm{~W} \\
\text { exposure: .1s- } \\
\text { inf }\end{array}$ & 3 & $\begin{array}{l}3 \times 1 \\
\mathrm{~cm}^{3}\end{array}$ & 4 & $\begin{array}{l}0.1 \mathrm{~m} \text {-inf } \\
\text { (further } \\
\text { with } \\
\text { longer } \\
\text { exposure) }\end{array}$ & 3 & $\begin{array}{l}\text { Superior } \\
\text { to stereo } \\
\text { or } \\
\text { structured } \\
\text { light }\end{array}$ & 3 & \\
\hline $\begin{array}{l}\text { ToF } \\
\text { Camera }\end{array}$ & 4 & $\begin{array}{l}>10 \mathrm{~W} \\
\text { exposure: } \\
\text { flash }\end{array}$ & 2 & $\begin{array}{l}100 \\
\mathrm{~cm}^{3}\end{array}$ & 2 & $\begin{array}{l}0.1 \mathrm{~m}- \\
10 \mathrm{~m}\end{array}$ & 4 & & 3 & \\
\hline $\begin{array}{l}\text { Spinning } \\
\text { Lidar }\end{array}$ & 1 & $\begin{array}{l}10 W \\
\text { scan: } \\
5 s\end{array}$ & 1 & $\begin{array}{l}100 \\
\mathrm{~cm}^{3}\end{array}$ & 3 & $\begin{array}{l}0.1 \mathrm{~m}- \\
30 \mathrm{~m}\end{array}$ & 4 & & 1 & $\begin{array}{l}\text { mechanical } \\
\text { sensitivity? }\end{array}$ \\
\hline
\end{tabular}




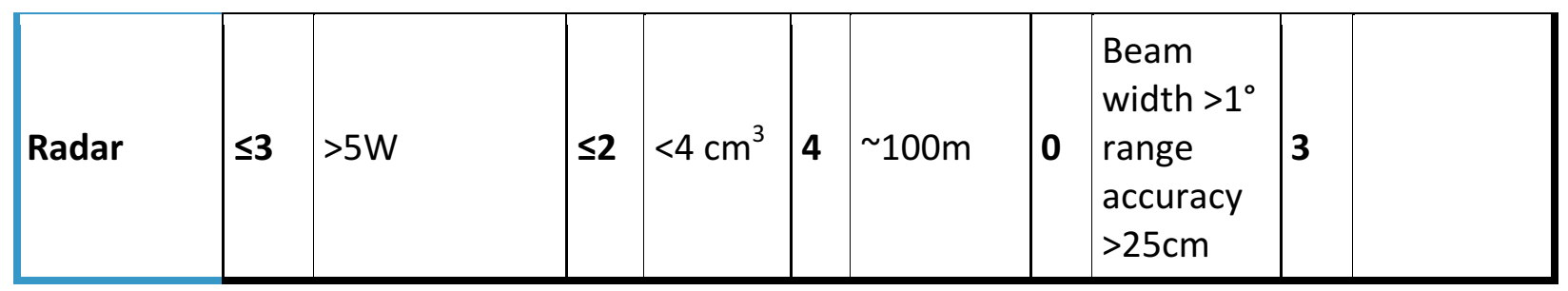

Figure 39: Qualitative evaluation of range-sensing devices. Rankings are on a 0-4 scale. See text for details.

\subsection{Science Instruments}

EVE will perform astrobiology and volcanology observations in the vent-conduit system, with the grand goal being the detection of life. Like Viking and Europa Lander, no singular measurement would provide sufficient evidence for the detection of life; rather, the conclusion that evidence of life had been detected would require multiple lines of evidence, from different instruments, on a set of samples examined across a variety of spatial scales (Hand, et al., 2017). However, the payload volume of DM is so limited that all the variety of instruments necessary to detect life is not likely to be accommodated in a DM. Therefore, we will take an alternative approach where instruments are distributed between multiple in-situ science DMs. In addition, sample return DMs will bring ice, liquid, and dust sample to SM, where various elaborate analyses are performed.

\subsubsection{Instruments on In-situ Science DM}

The payload space in DM is extremely limited. Our standard three-section design (see Section 2.1.1) accommodates science instruments in its middle section. Its internal volume is approximately $5 \mathrm{~cm} \times 5 \mathrm{~cm} \times 10 \mathrm{~cm}$. DM can have additional instrument sections (like a snakebot), but the volume of each section will be limited anyway. A good news is that, because the interior of the vent-conduit system is almost completely unknown and orbital reconnaissance is impossible, even very simple measurements could significantly advance scientific knowledge. Below is a partial list of science instruments that can be miniaturized and fit in an in-situ science DM.

\subsubsection{Volcanology package: temperature, pressure, and flow speed}

Measurement of temperature, pressure, and flow speed in the vent-conduit system, combined with 3D mapping provided by the perception system (Section 4.5), will significantly advance the understanding of the eruption mechanism. Off-the-shelf miniaturized temperature sensors are available. A miniaturized pitot tube can measure static and dynamic pressures, and the flow speed can be estimated from the difference of them. This package is likely to occupy only a fraction of the payload volume; hence it can be a standard package for all DMs. 


\subsubsection{Habitability package: pH, salinity, and oxidation-reduction potential (ORP)}

$\mathrm{pH}$, salinity, and oxidation-reduction potential (ORP) are three of the most important indicators for habitability, all of which can be measured using off-the-shelf miniaturized devices. Sometimes, $\mathrm{pH}$ is even an indicator of extant life. For example, a significant change in $\mathrm{pH}$ (from 9.2 to 0 ) over just a few km in range, observed in a sulfuric acid cave, Cueva de Villa Luz cave, Tabasco, Mexico, is due to the activities of microbial lives (Boston, 2016).

Commercially available potentiometric devices can operate with a very small volume of liquid sample. If DM can reach the liquid interface, it can directly measure the $\mathrm{pH}$ of the body of water. Alternatively, it can operate before reaching the liquid interface by collecting liquid particles in the flow with an absorbent material.

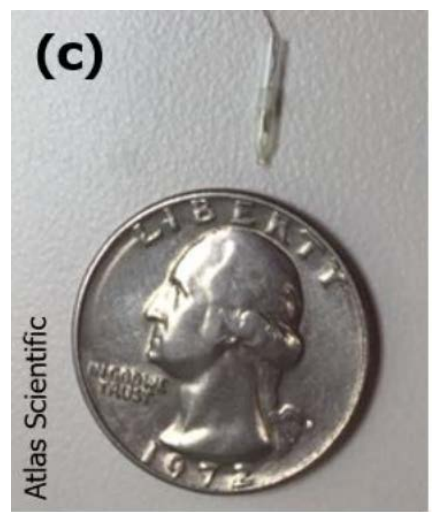

Figure 40 Miniature pH probe. Image take from (Kehl, et al., 2016)

\subsubsection{Microscopic imager}

Imaging, both macroscopic and microscopic, is an effective tool for detecting life. At a macroscopic scale, microorganisms frequently produce distinctive "biovermiculation patterns," as shown in Figure 41, which could be a universal signature of life (Boston, 2016). At a microscopic scale, life often creates distinctive structures, as in Figure 42.

Macroscopic patterns can be identified with DM's stereo cameras, while finding microscopic patterns requires a dedicated microscopic imager. Combination of macroscopic and microscopic imaging with UV LED would be useful as many lifeforms on Earth exhibit UV fluorescence.

Microscopic imager is a relatively small instrument. For example, Mars Hand Lens Imager (MAHLI) (Figure 43), the microscopic imager mounted on the robotic arm of Curiosity, is $\sim 4 \mathrm{~cm}$ in width. Miniaturizing it for DM would not be a major challenge. However, DM would not be able to accommodate a mechanism for a complex sample preparation. Therefore, its use would be limited to imaging of the raw surface of ice walls as well as liquid water. Microscopic observation that requires sample preparation is performed in SM on samples brought by sample return DMs. 

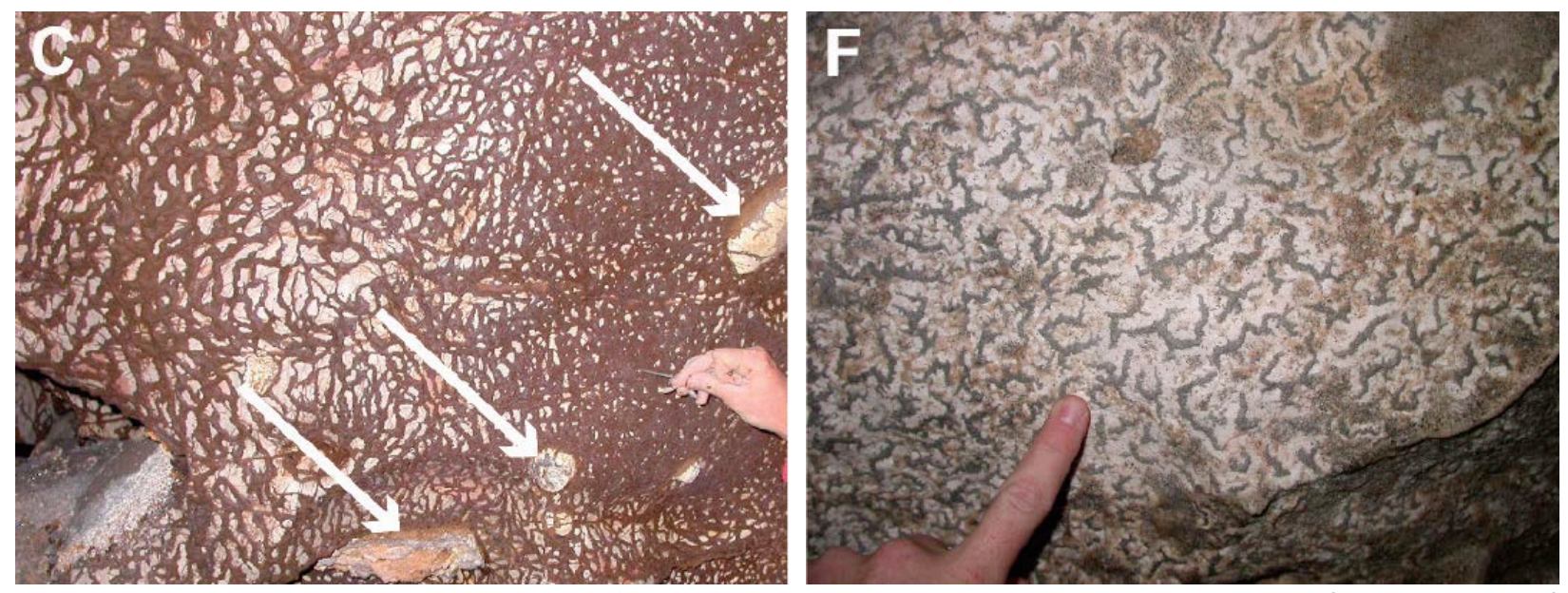

Figure 41 Macroscopic biovermiculation patterns found in the Frasassi cave system, Italy. Images taken from (Jones, et al., 2008)
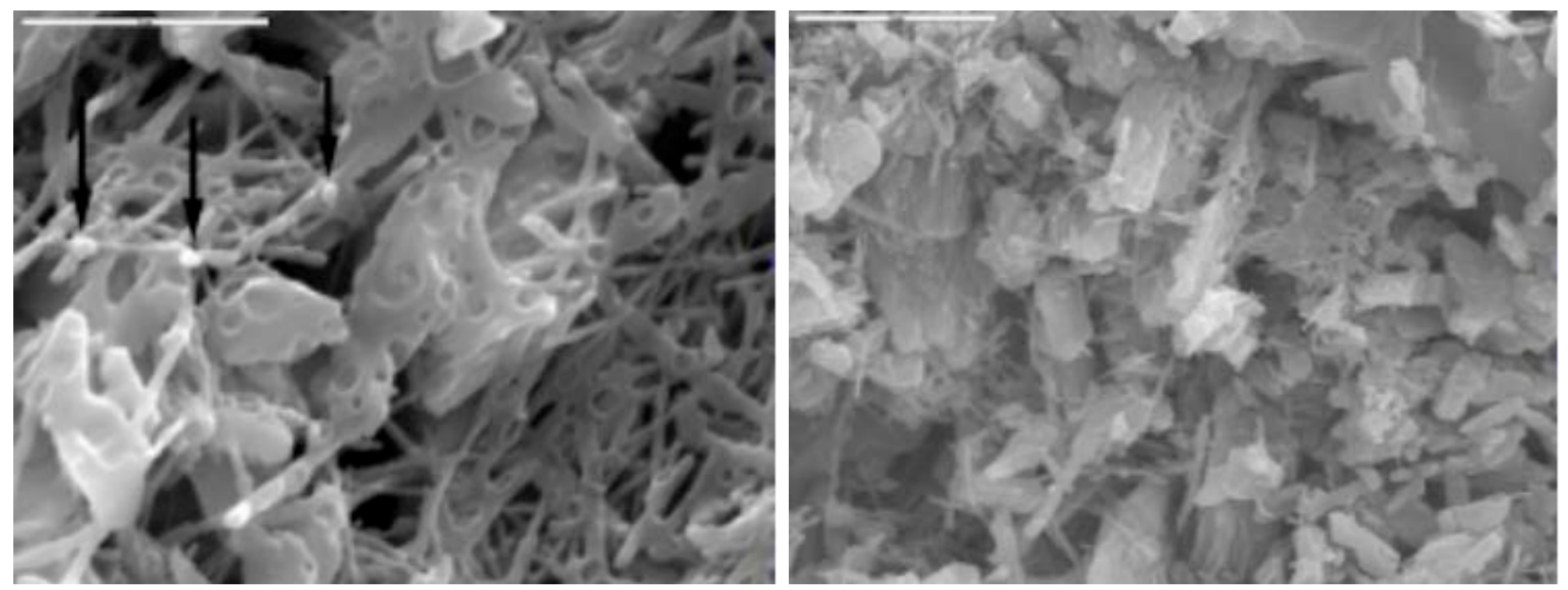

Figure 42 Microscopic structures created by microbial life. Left: biologically active calcite moonmilk (Crisco), Spider Cave, NM. Right: Fossil microbial mat textures, Hidden Cavem NM. Scale bar $=2 \mu \mathrm{m}$. Images are taken from (Boston, et al., 2001) 


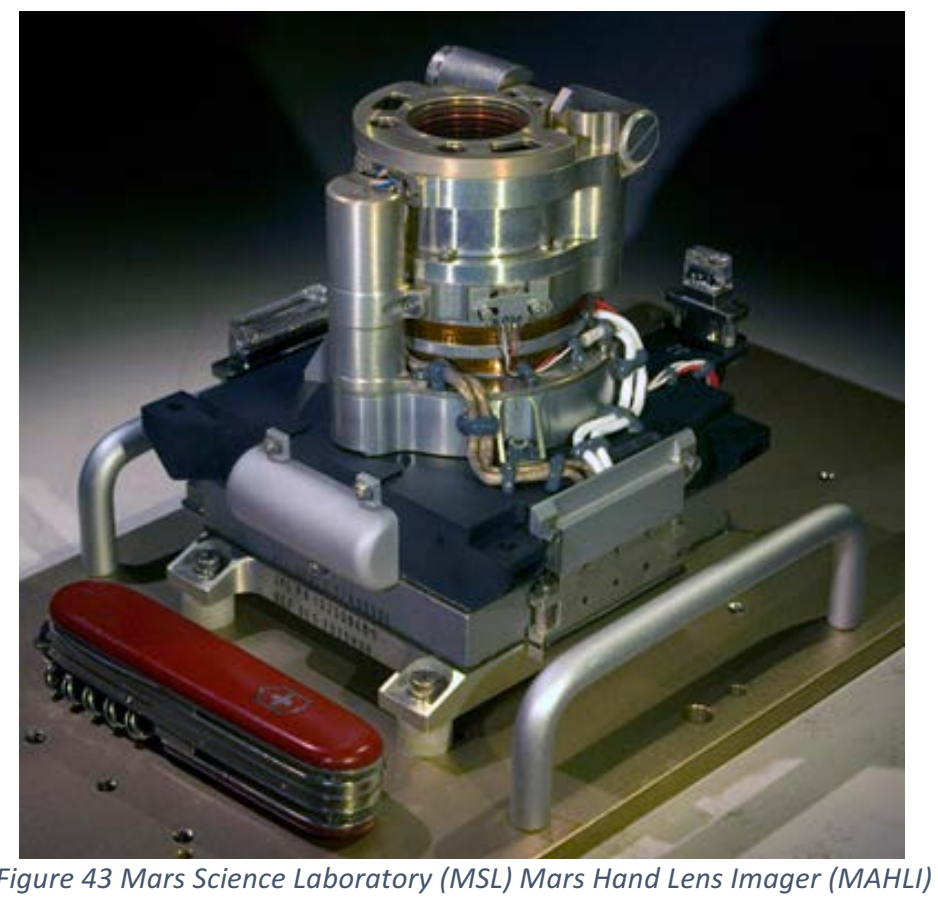

\subsubsection{Life detection package: microchip electrophoresis with laser-induced fluorescence}

Microchip electrophoresis (ME) is a miniaturized variant of capillary electrophoresis (CE) implemented on a microfluidic device. ME does not require the use of high-pressure pumps or packed columns of separation media (Willis, et al., 2015). Coupled with laser-induced fluorescence (LIF), it can measure the distributions of molecular properties in organic molecules, including carboxylic acids and amino acids. Such measurement provides a practical way to search for life based on Lego principle (McKay, 2004). Like LEGO blocks, life is composed of a subset of naturally available materials. For example, terrestrial life uses 20 amino acids, whereas over 70 have been detected in meteorites (Willis, et al., 2015). Therefore, biotic sample exhibits distinct distribution of molecular properties from that of abiotic samples (Figure 44).

Various prototypes of end-to-end instrument have been developed at JPL, as shown in Figure 45. They are tens of $\mathrm{cm}$ in size, but most of the volume is typically occupied by liquid extraction and sample handling systems while the detector (microfluidic chips) itself is a few $\mathrm{cm}$ in size (Figure 46). If DM can reach the liquid interface and directly intake liquid (as shown in Figure 47), or alternatively collect liquid samples in the vent-conduit system, the liquid extraction system is unnecessary, and the sample handling system can be simplified significantly. 

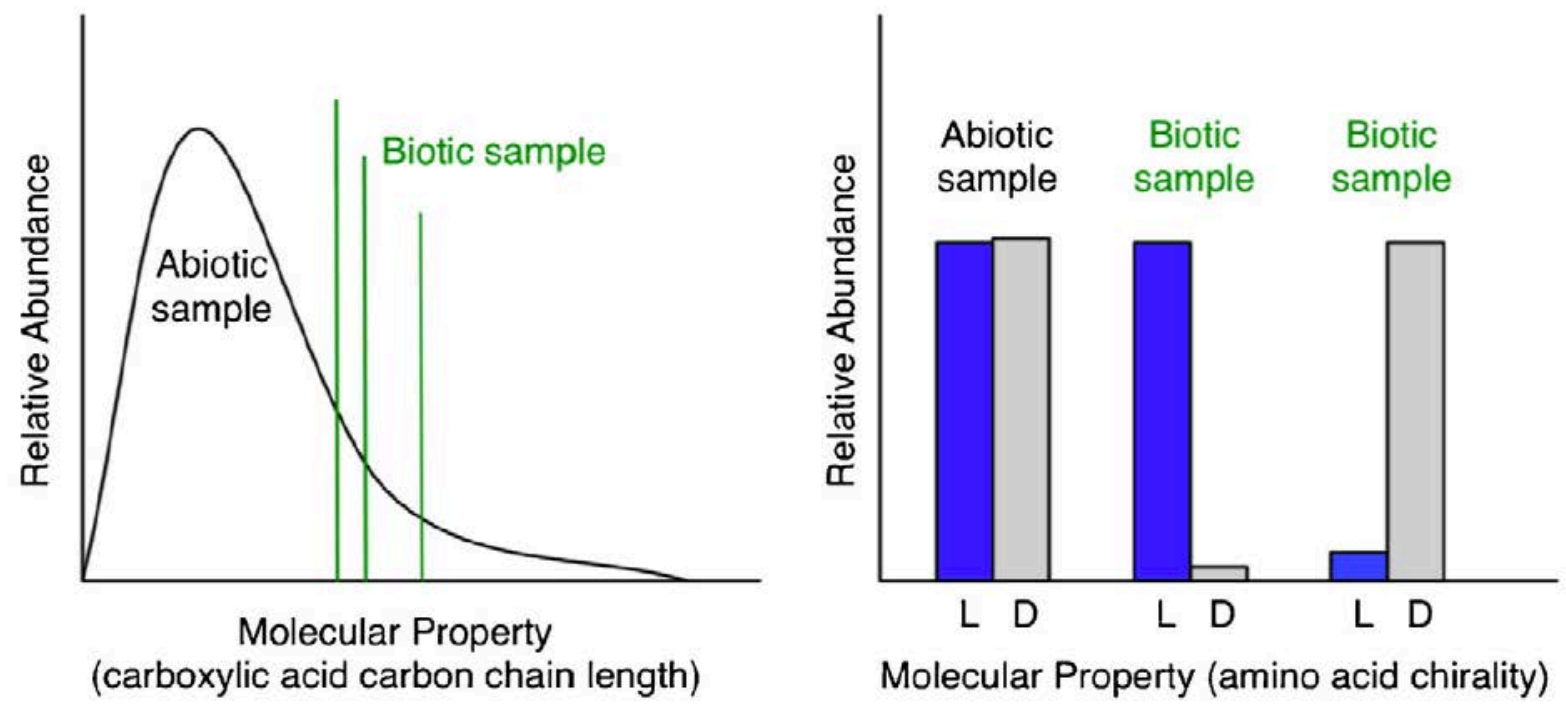

Figure 44 The Lego principle The Lego principle illustrates a practical way to search for evidence of life and biological processes. Left - from (McKay, 2004), right - from (Willis, et al., 2015).
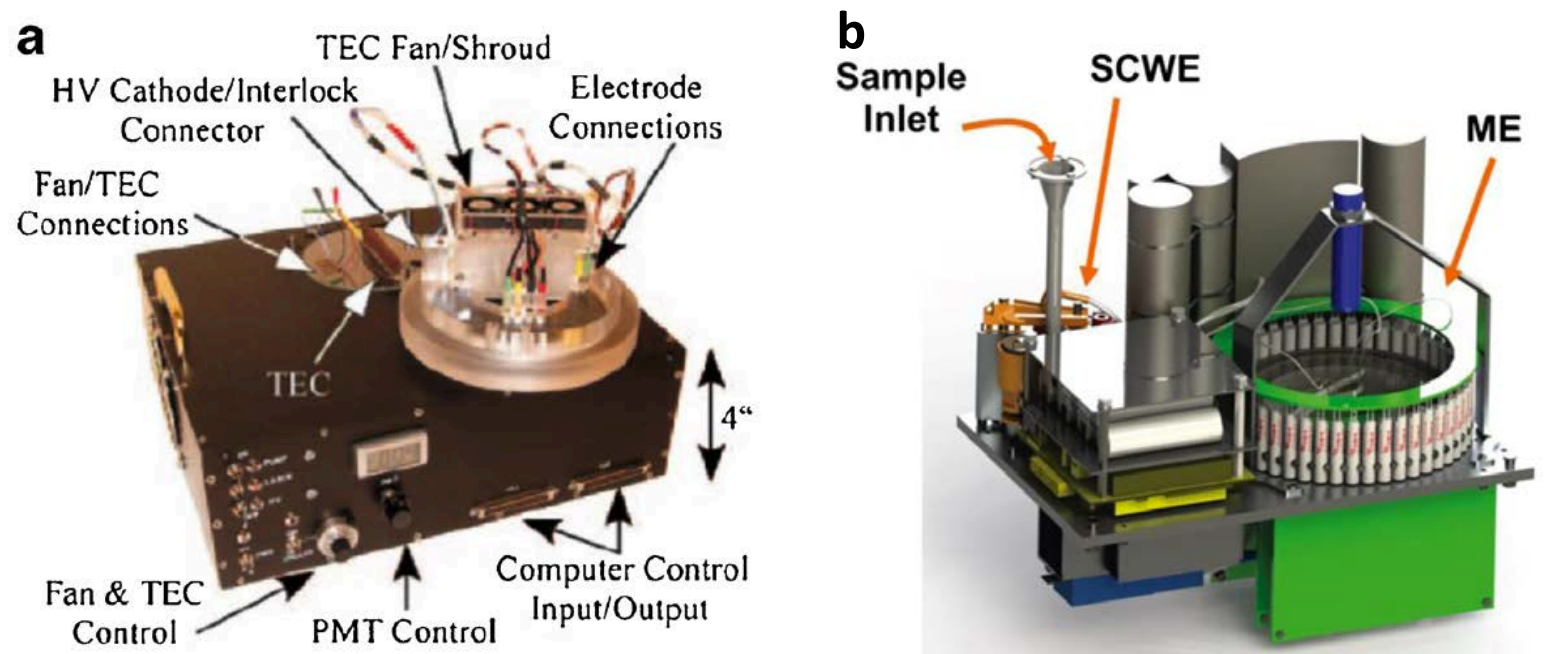

Figure 45 Prototypes of end-to-end life detection instruments. a: The Mars Organic Analyzer, b: Planetary In Situ Capillary Electrophoresis System (PISCES). Images take from (Willis, et al., 2015). 
a

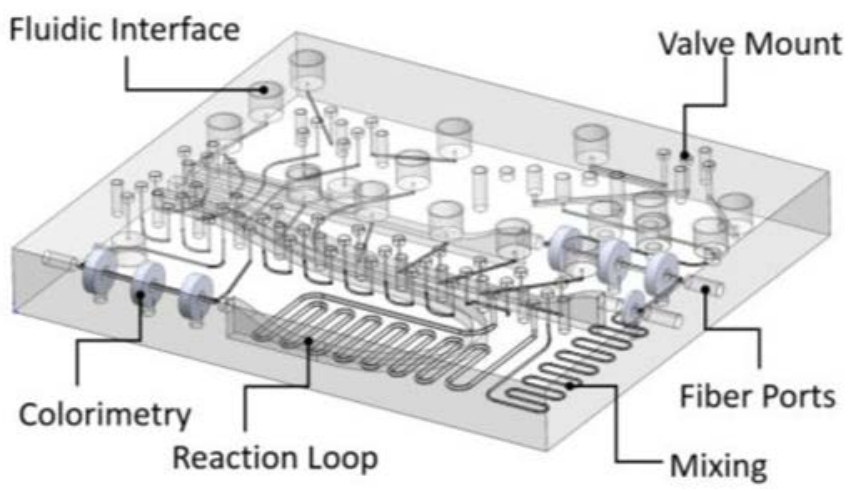

b

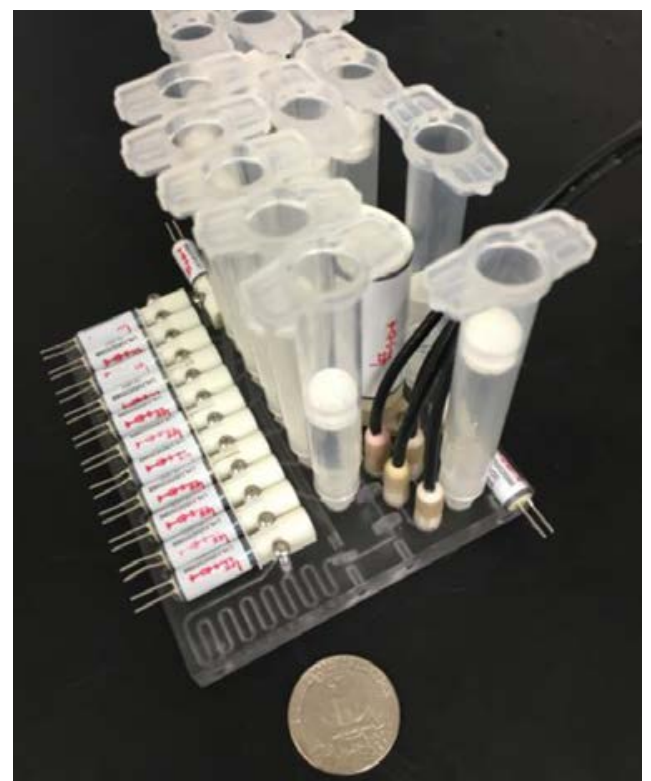

Figure 46 An example of microfluidics chip. a: 3D printed MicroFIA manifold, b: assembled MicroFIA system. Images taken from (Kehl, et al., 2016).

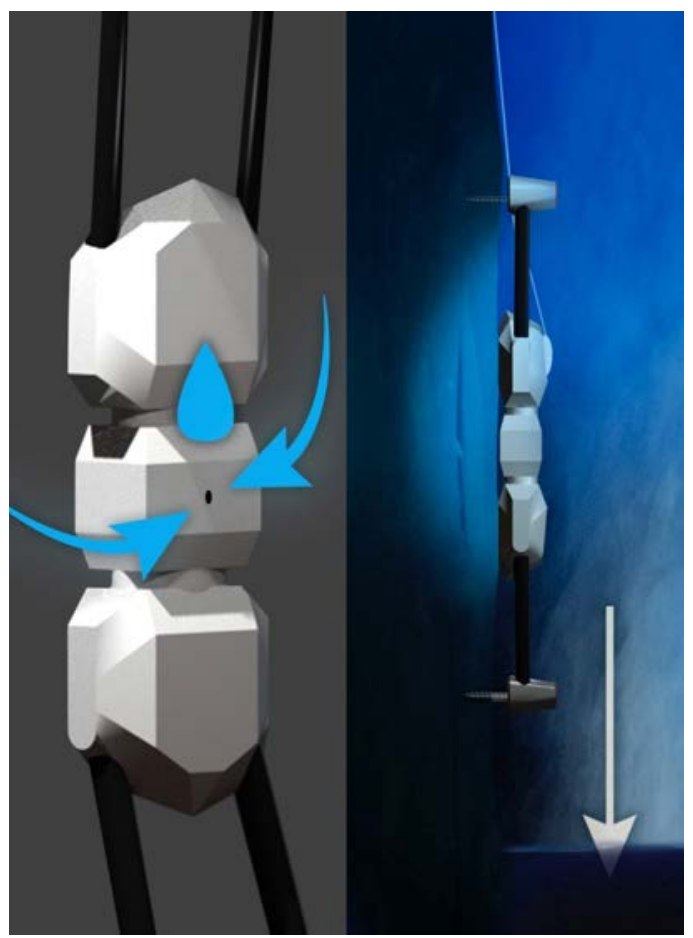

Figure 47 In-situ science DM with microfluidics chip. It directly intakes liquid sample upon the arrival at liquid interface. 


\subsubsection{Instruments on sample return DM}

Ice screw creates an ice core when screwed into ice. Therefore, a DM produces ice cores at every step. One idea is to store some of the ice cores in the middle section and bring them back to SM. However, transferring ice cores from the end effector to the middle section would require additional mechanical system. If multiple samples are stored, the sample handling system typically needs a revolver-like mechanism (Figure 45-b) which is very bulky.

A more realistic idea would be to install a dedicated ice screw for sampling in the middle section, as shown in Figure 48. In order to minimize the complexity of sample handling system, the sample return DM is designed to collect only one ice core per trip. Once it cores the ice, it seals the ends of the screw and bring the core back. It can make another trip to collect more samples. Alternatively, if space permits, a DM can have multiple ice screws in the middle section.

The volume of an ice core produced by an $8-\mathrm{mm}$ ice screw with $2.5 \mathrm{~cm}$ insertion length is approximately $3 \mathrm{cc}$. This is comparable to the required volume of sample for Europa Lander's instruments ( $5 \mathrm{cc}$ for Microscope for Life Detection, $1 \mathrm{cc}$ for Organic Compositional Analyzer (mass spectrometer) and Vibrational Spectrometer) (Hand, et al., 2017).

Other types of sample return DM include liquid sample collector and dust particle collector.

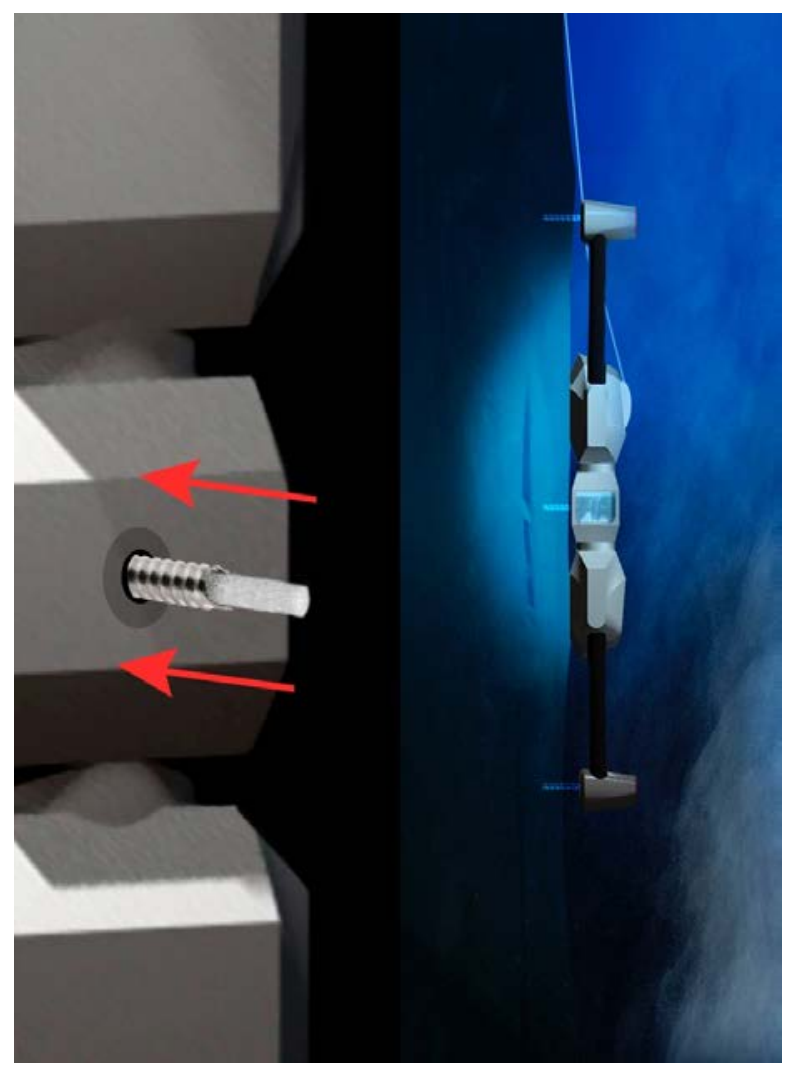

Figure 48 Sample return DM equipped with the fifth ice screw for sampling in its middle section. 


\subsubsection{Instruments on SM}

SM can accommodate instruments that are too voluminous for a DM. SM's instruments are used for for analyzing the samples brought back by sample return DMs. One of the most valuable types of instrument for detecting life would be a resolution mass spectrometer. Microscopic imager and vibrational spectrometer, like the ones on the model payload of Europa Lander, would also be valuable. 


\section{Planetary Protection}

Following Cassini's discovery of water plumes, Committee on Space Research (COSPAR) Planetary Protection Policy was amended to add Enceladus to the list of target bodies for PP classification Category III/IV, and NASA's planetary protection policy (NPR 8020.12D, Planetary Protection Provisions for Robotic Extraterrestrial Mission) was also updated as such. As EVE will land and potentially impact the surface of Enceladus, it should be compliant with the Category IV requirements. Specifically, "the probability of inadvertent contamination of an ocean or other liquid water body" must be reduced to less than $1 \times 10^{-4}$ per mission (NPR 8020.12D, Sec. 5.4).

As EVE's DM could directly contact the liquid body of water in a vent-conduit system, significantly more elaborate bioburden reduction processing would be necessary than landing missions such as Europa Lander. A similar level of bioburden reduction processing would be necessary for SM as well because 1) DMs will be in contact with SM over a long period and 2) upon a failed landing or spacecraft disintegration, SM's RTGs could melt through the ice shell and reach the subsurface ocean ${ }^{10}$.

A comparable mission concept is the mechanical ice probe proposed by (Wilcox, et al., 2017), which uses RTG and General Purpose Heat Source (GPHS) modules containing Pu-238 (mode details are in Section 2.3.2). They propose heat sterilization at $500 \mathrm{C}$ for significant periods of time because "exposure to $500 \mathrm{C}$ for extended durations should pyrolize any organic material, decomposing all large organic molecules (Wilcox, et al., 2017)." However, further studies should be done to prove the particular sterilization method can achieve the $1 \times 10^{-4}$ contamination probability requirements. Building a spacecraft to tolerate the extensive heat sterilization would be a major technical challenge. In addition to heat sterilization, development of alternative bioburden reduction technologies, including chemical- and radiation-based ones, should be promoted.

\footnotetext{
${ }^{10}$ There is a misconception that RTG is not allowed on icy moons. There is no such rule in COSPER's and NASA's planetary protection policy. However, because RTG can melt through the ice shell over a long period and reach the ocean, it significantly increases the probability of transferring microbes from the spacecraft to the ocean ( $P_{S}$ and $P_{R}$ in the Coleman-Sagan equation), compared to a non-RTG surface mission with the same level of bioburden reduction. The increase in probability can be offset by reducing the number of microbes on the spacecraft ( $N_{0}$, in the Coleman-Sagan equation), which in turn can be achieved by more stringent sterilization.
} 


\section{Experimental Studies}

Because ice screws are intended for ice climbing, two pieces of quantitative information needed for system trade study in Section 4.2 were not available. One is the energy needed for inserting and removing screw, and the other is the maximum sheer force the anchor can withstand. The first item is not available probably because how many joules needed for operating an ice screw is largely irrelevant for human operators. As for the second item, only the resistance to normal force is found in existing analysis.

In order to find the two critical pieces of information, we performed a series of experiments in an ice chamber at JPL.

\subsection{Energy required for ice screw insertion and removal}

We performed experiments in order to determine the approximate energy required to insert a 10 -cm ice screw into $\mathrm{H}_{2} \mathrm{O}$ ice. The screw we used is $10 \mathrm{~cm}$ in length and $1.6 \mathrm{~cm}$ in diameter, 118 $\mathrm{g}(4.2 \mathrm{oz}$.) in weight, with $10 \mathrm{kN}$ rated strength, manufactured by Black Diamond.

The resultant data yielded a maximum torque of $3.619 \mathrm{Nm}$ on the ice screw in a $-20 \mathrm{C}$ thermal chamber. The maximum torque required to remove the ice screw from the ice in the $-20 \mathrm{C}$ thermal chamber was $1.634 \mathrm{Nm}$. A maximum torque of $3.946 \mathrm{Nm}$ on the ice screw was seen in the 'warm' room (room-temperature). Lastly, a minimum normal force of $6 \mathrm{~N}$ was required to begin insertion for the ice screw, provided there was rigidity and stabilization provided to the screw. From these results, we derived a conservative energy requirement estimate of insertion and removal of the ice screw came out to $495.4 \mathrm{~J}$. The process to determine these values is described below.

\subsubsection{Thermal Chamber Experiments (-20 C)}

During this experiment, the minimum required normal force to begin insertion was analyzed, the minimum removal torque was analyzed, and the required energy for insertion due to the required torque to turn the screw. The torque was determined by measuring with a force gauge the force perpendicular to the ice screw handle required to insert the screw at a distance of 72 $\mathrm{mm}$ from the center of the screw.

\subsubsection{Maximum Torque Experienced during Insertion on the Ice Screw \& Corresponding Energy Requirement in the Thermal chamber (-20 C)}

In the thermal chamber, the maximum torque that was required to insert the screw at any given point was $\tau=3.619 \mathrm{Nm}$. (See Figure 2, for the complete set of torque data.) The approximate maximum energy required to insert the screw due to torque can be calculated by the equation $2 \pi N \tau=E$, where $N=$ number of threads on screw, $E=$ energy required for insertion due to torque $[J]$. The assumption that the max torque is applied for the entire insertion of the screw is conservative, and would result in $E=2 \cdot \pi \cdot 15$ threads $\cdot 3.619 \mathrm{Nm} \approx 341.2 \mathrm{~J}$. 


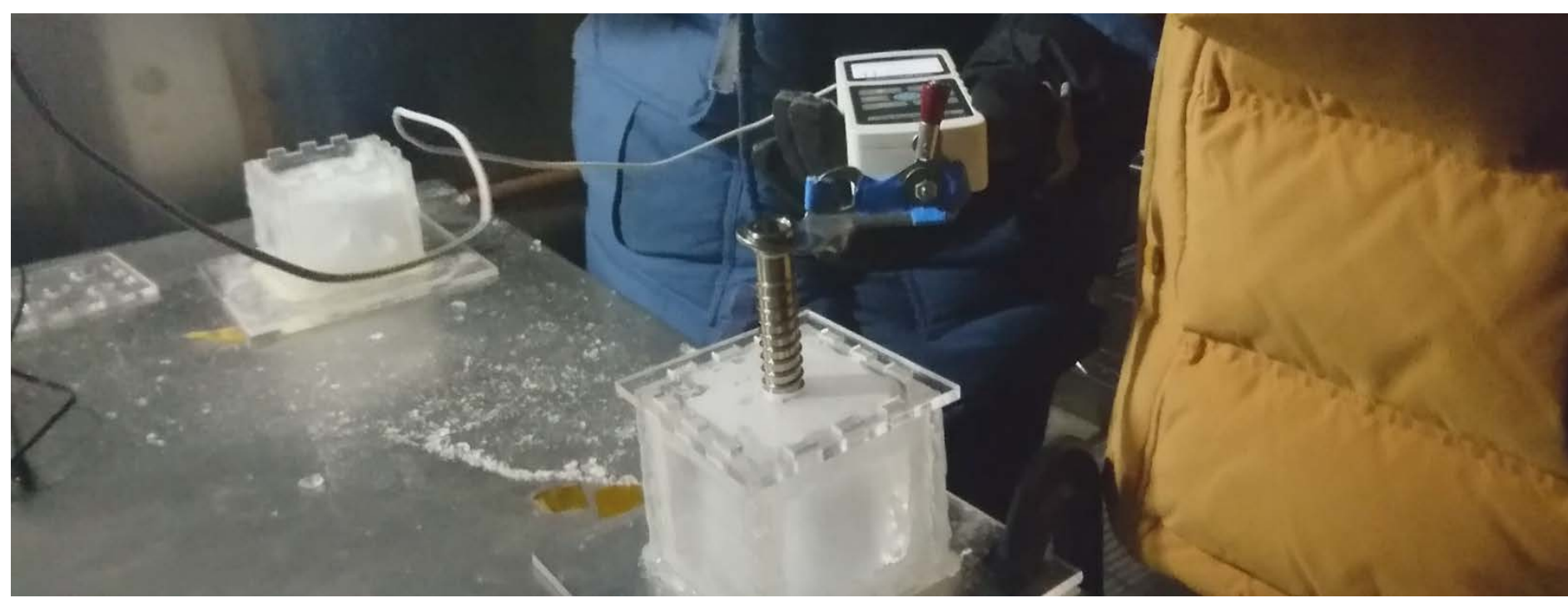

Figure 49 - The maximum torque measured during the insertion of the ice screw was $3.619 \mathrm{Nm}$. In order to get this number, the force gauge measured the perpendicular force to the ice screw at a distance of $72 \mathrm{~mm}$ from the center of the ice screw. If this maximum torque is assumed to be the torque required during the entire insertion of the screw, it would require about $341.2 \mathrm{~J}$ of energy.

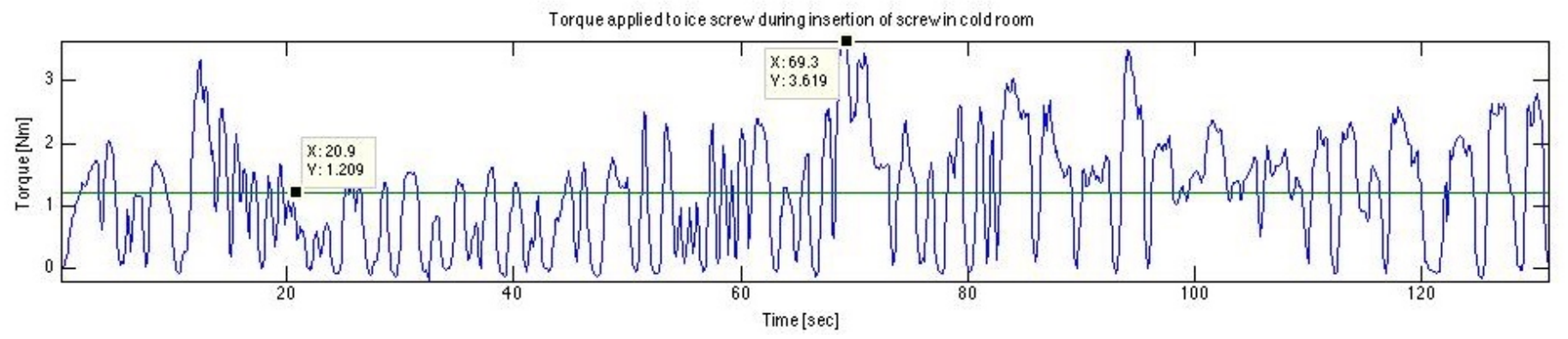

Figure 50 - The torque applied to the ice screw during insertion as a function of time is shown in blue. The green line depicts the average of the data over the entire timeframe $(1.209 \mathrm{Nm})$. As shown in this graph, the torque often drops to zero. The reason for these drops is that during the experiment, there were several cables connected to the force gauges, and as a result the ice screw insertion and removal was frequently halted to allow for repositioning of the cable or repositioning of the individual's hands who were holding the force gauge.

\subsubsection{Maximum Torque Experienced during Removal on the Ice Screw \& Corresponding Energy Requirement in the Thermal chamber (-20 C)}

In the thermal chamber, the maximum torque that was required to remove the screw at any given point was $\tau=1.634 \mathrm{Nm}$. The approximate maximum energy required to remove the screw due to torque can be calculated by the equation $2 \pi N \tau=E$, where $N$ =number of threads on screw, $E=$ energy required for removal due to torque $[J]$. The assumption that the $\max$ torque is applied for the entire removal of the screw is conservative, and would result in $E=2$. $\pi \cdot 15$ threads $\cdot 1.634 \mathrm{Nm} \approx 154.0 \mathrm{~J}$. See Figure 2 , for the complete set of torque data. 


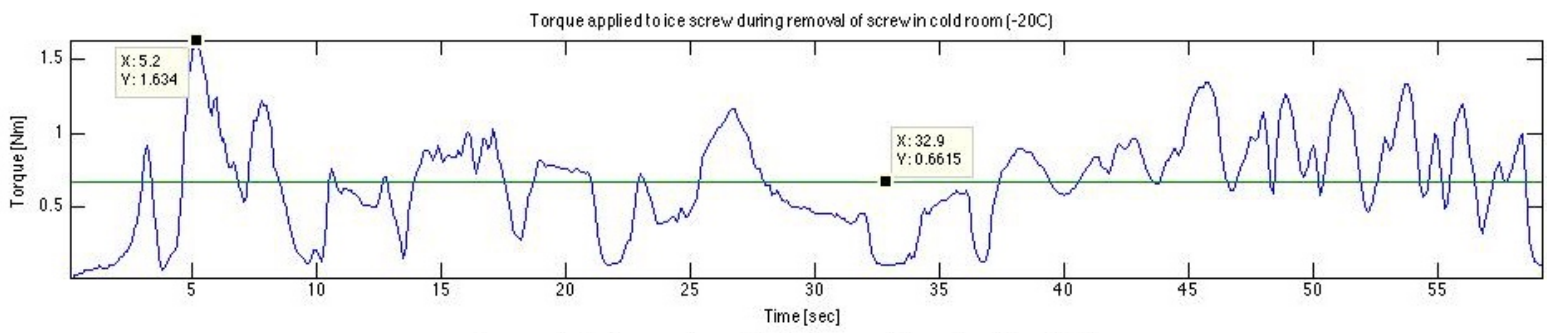

Figure 51- The torque applied to the ice screw during removal in the thermal chamber as a function of time is shown in blue. The green line depicts the average of the data over the entire timeframe $(.6615 \mathrm{Nm})$. As shown in this graph, the torque often drops to zero. The reason for these drops is that during the experiment, there were several cables connected to the force gauges, and as a result the ice screw insertion and removal was frequently halted to allow for repositioning of the cable or repositioning of the individual's hands who were holding the force gauge.

\subsubsection{Minimum Required Normal Force to begin Insertion}

In order to analyze the minimum required normal force we applied a series of normal forces on the ice screw. For each normal force, we tested whether or not it was sufficient normal force for the ice screw to begin insertion. Successful insertion was loosely defined as the point after which the screw could continue to be screwed into the wall using only torque. (In layman's terms, we were trying to measure the force an ice climber would have to push into the ice wall to ensure the threads and teeth of the ice screw caught as he was inserting the screw).

We tested a weight on bit (wob) force of $\approx 21 N, \approx 11 N, \approx 6 N$ and $\approx 3 N$ with a confidence interval of about $\approx .4 N$. The screw successfully began insertion for $21 N, 11 N$, and $6 N$. The screw had some problems inserting when only $3 N$ of normal force were applied, and it slipped frequently on the ice between teeth catching occasionally on the ice.

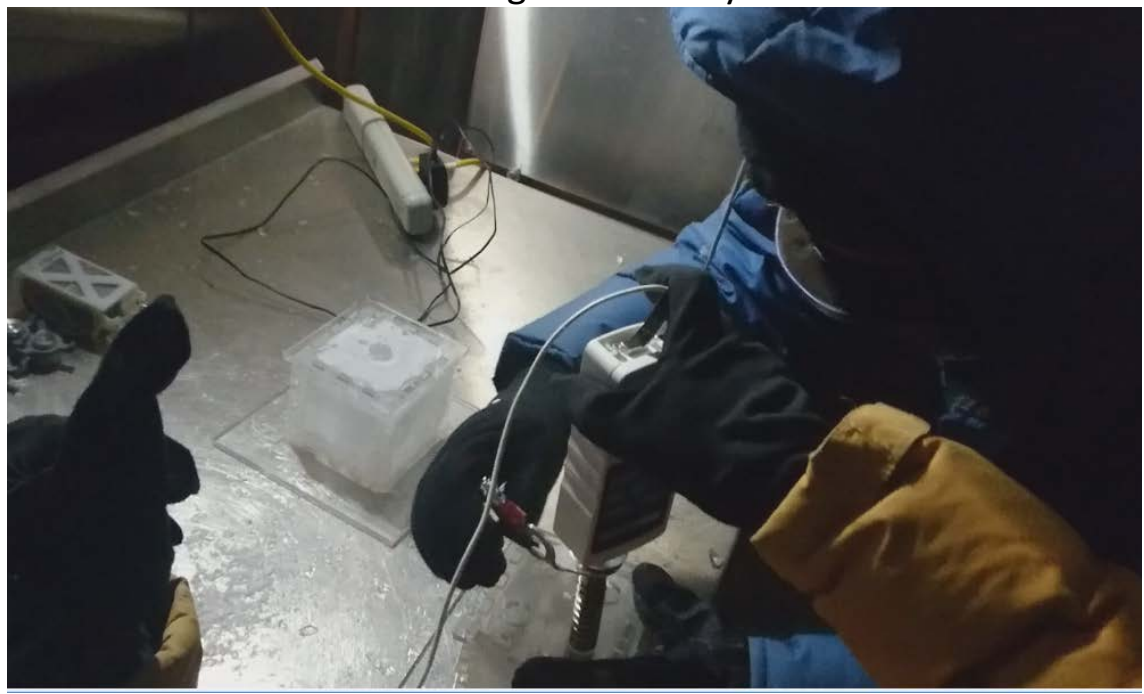

Figure 52 - Required normal force to begin insertion of the ice screw was measured with a force gauge by Aaron Curtis. About 6 $N$ appeared to be the minimum required force.

If a conservative assumption is used, we can calculate the total energy required to apply the downwards force. If this force needs to be applied to the first $20 \mathrm{~mm}$ of insertion of the ice screw (consistent with the experiment), and a conservative10 $\mathrm{N}$ of normal force is assumed, then the energy requirement would be $0.2 \mathrm{~J}$. 
This experiment shows that the energy requirement for pre-loading is insignificant compared to the energy required for turning the screw into the, since the insertion and removal power due to torque is on the order of hundreds of Joules. The energy required to provide an adequate normal force for $20 \mathrm{~mm}$ is on the order of tenths of a Joule.

One important thing to note is that there was added constraint on the ice screw due to the hole in the lid that added stability to the insertion process. During insertion, if the ice screw did not have a stabilizing force to prevent it from slipping, these normal forces would not accurately reflect the required normal force. This was provided by the lid of the box, which had a hole which tightly fit the screw, as well as potentially some stabilizing force from human operator' hands.

\subsubsection{Conservative energy requirement estimate for the Insertion and Removal of the Ice Screw}

In conclusion, if the maximum torque for removal and insertion are used as well as a conservative normal force is used, the energy requirement would be $E_{\text {total }}=E_{\text {insertion }}+$ $E_{\text {normal }}+E_{\text {removal }}=341.2 \mathrm{~J}+0.2 \mathrm{~J}+154.0 \mathrm{~J}=495.4 \mathrm{~J}$.

\subsubsection{Room Temperature Experiment}

The same series of experiments were performed for insertion of the ice screw in room temperature. The purpose of the room temperature experiment was to determine if melting due to the ambient temperature affected the total energy required to insert the screw. During the experiment, the ice screw was initially cooled down to $-17 \mathrm{C}$, the same temperature of the ice. Gloves were worn when physically handling the ice screw in order to minimize heating due to conduction from the human body.

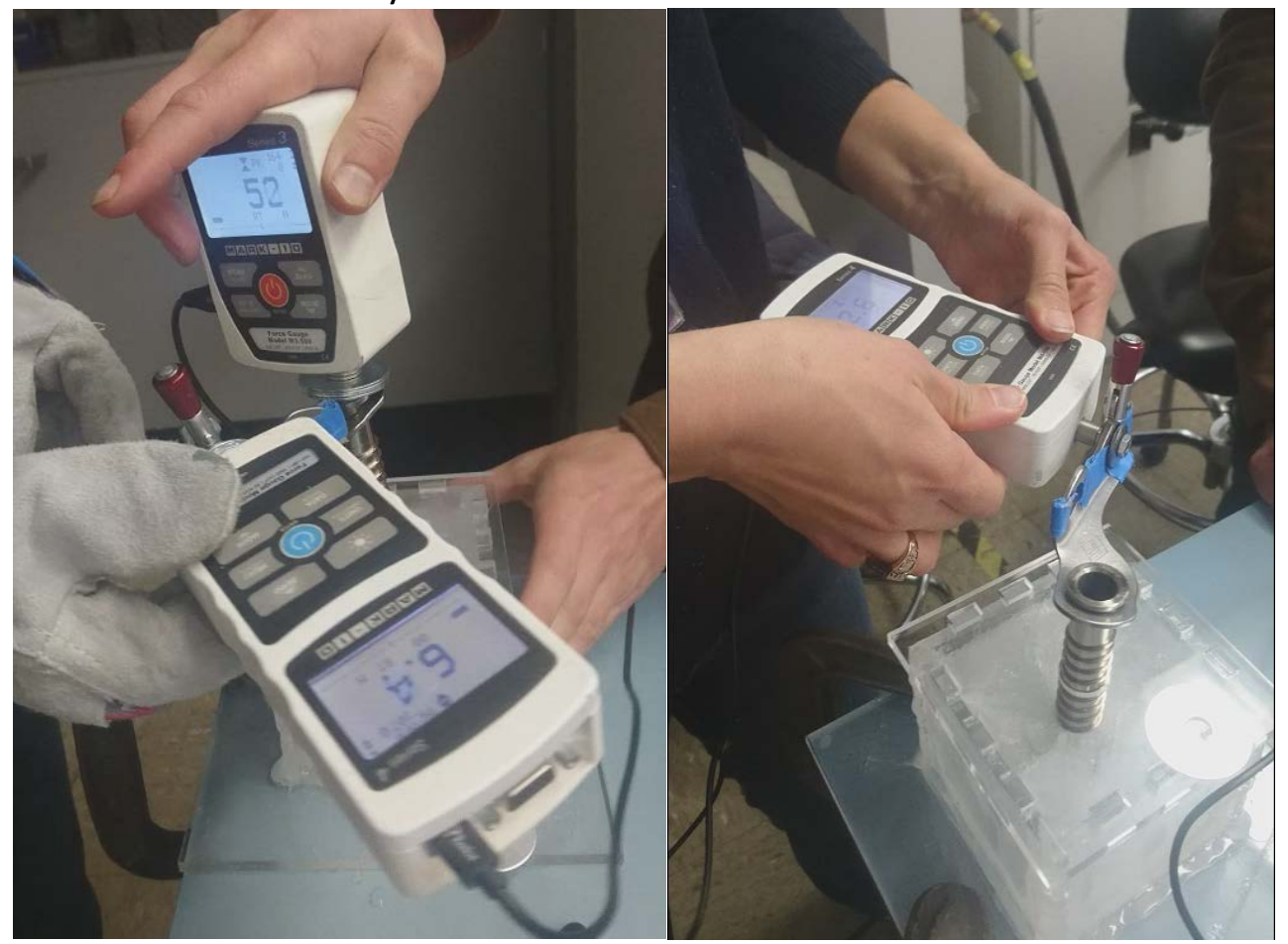


The data collected in the warm room proves to be very similar to the data collected in the thermal chamber, which suggests that slight warming due to ambient temperatures change the torque experienced by the ice screw only by about $\approx 10 \%$. These variations are likely due to normal variations in separate experiments. However, the results are still preliminary, hence more experiments would need to be performed in order to draw a conclusion that ambient temperature does in fact affect the required energy to insert the ice screw.
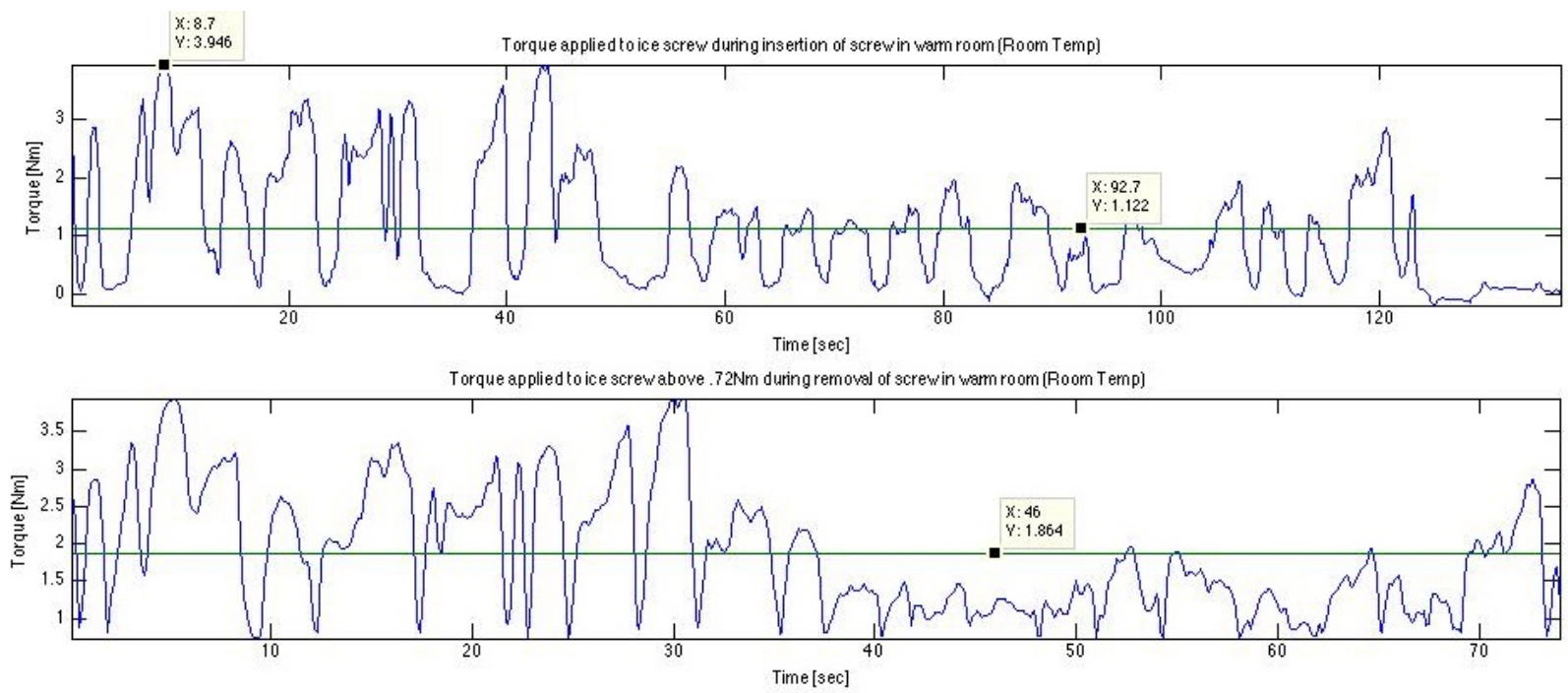

\subsubsection{Conclusion}

In conclusion, the energy required to insert an ice screw with $10 \mathrm{~cm}$ in length and $1.6 \mathrm{~cm}$ in diameter into $\mathrm{H} 2 \mathrm{O}$ ice at $-17 \mathrm{C}$ requires at most $~ 350 \mathrm{~J}$ per cycle. Additional experiments are planned to identify the dependency on the size of screw. The results will be used for system trade study.

\subsection{Ice screw's resistance to sheer force}

Previous studies on the same models of ice screw have highlighted several forces and resistance characteristics of the ice screws in uniform ice (Beverly, 2009). However, those studies mostly concentrated on normal forces and in the full use of the ice screw itself. The results show that a $10 \mathrm{kN}$ load can be safely achieved in nominal condition.

Our study is then interested in testing ice screws in a condition closer to our case, verifying the maximal shear force the ice and ice screw can withstand, whenever or not the ice screw incur in plastic deformation and the dependence of those factor with the used length of ice screw.

\subsubsection{Experiment Setup}

A pressing stand with a maximal load of 40 Kilo Pound was available for the experimental tests. Unfortunately, neither the advancement speed nor the dislocation were measurable, and as such the load history is relevant only on a qualitative level, and only the peak value of the forces is a reliable test result. Since the high forces involved for this test, the need of operating with water 
and temperatures it has been made necessary to create a custom-made container to freeze water in aluminum. Furthermore, a special attachment to load the screw was ordered to be produced trough CNC in Al 7075 T3.
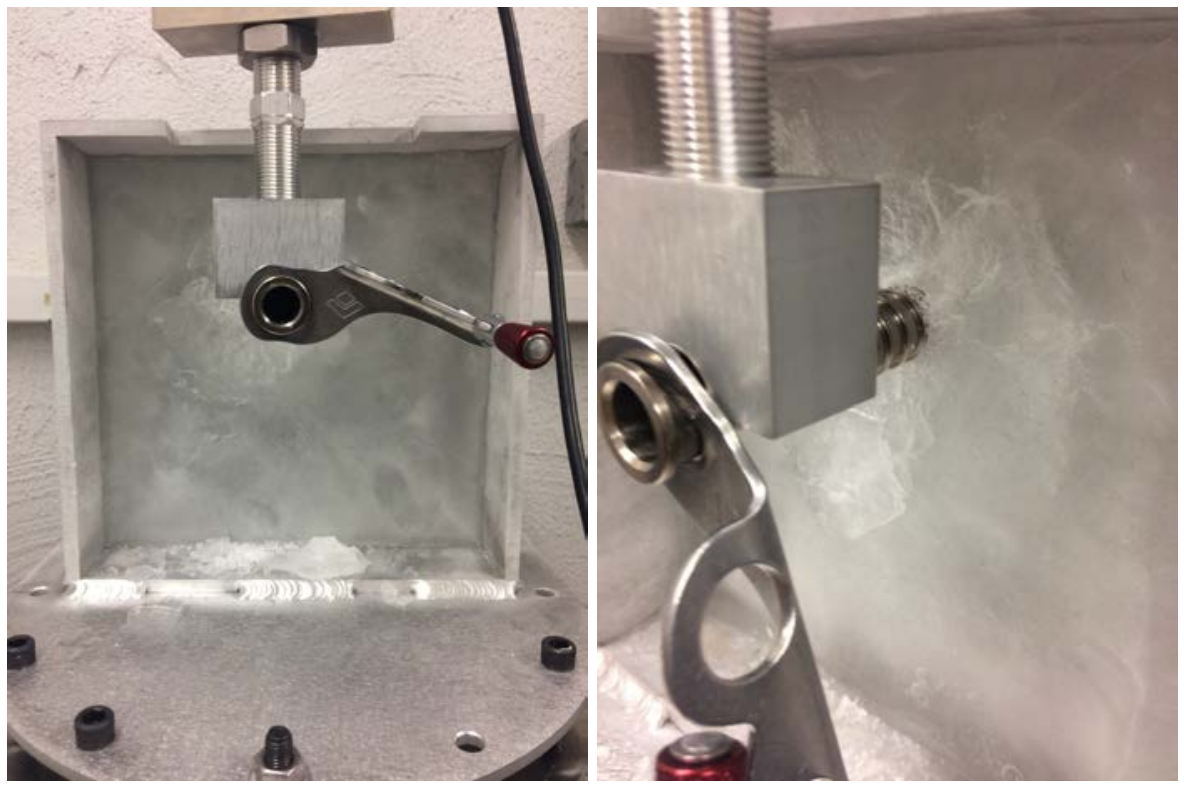

Figure 53 Experimental setup to measure the tolerable sheer force of ice screw. The ice screw is inserted to ice and pushed downwards by a pressing stand.

The container where then filled with water and kept for several days in the cold chamber at $10^{\circ} \mathrm{C}$. Before the test and after each test the surface ice and a substantial portion of the ice close to the ice-screw or that have shown fracture forming was removed a new water added and congealed.

\subsubsection{Results}

A total of 12 tests were performed. The chart below show the result distribution as a function of the length of ice screw inserted in the ice. 


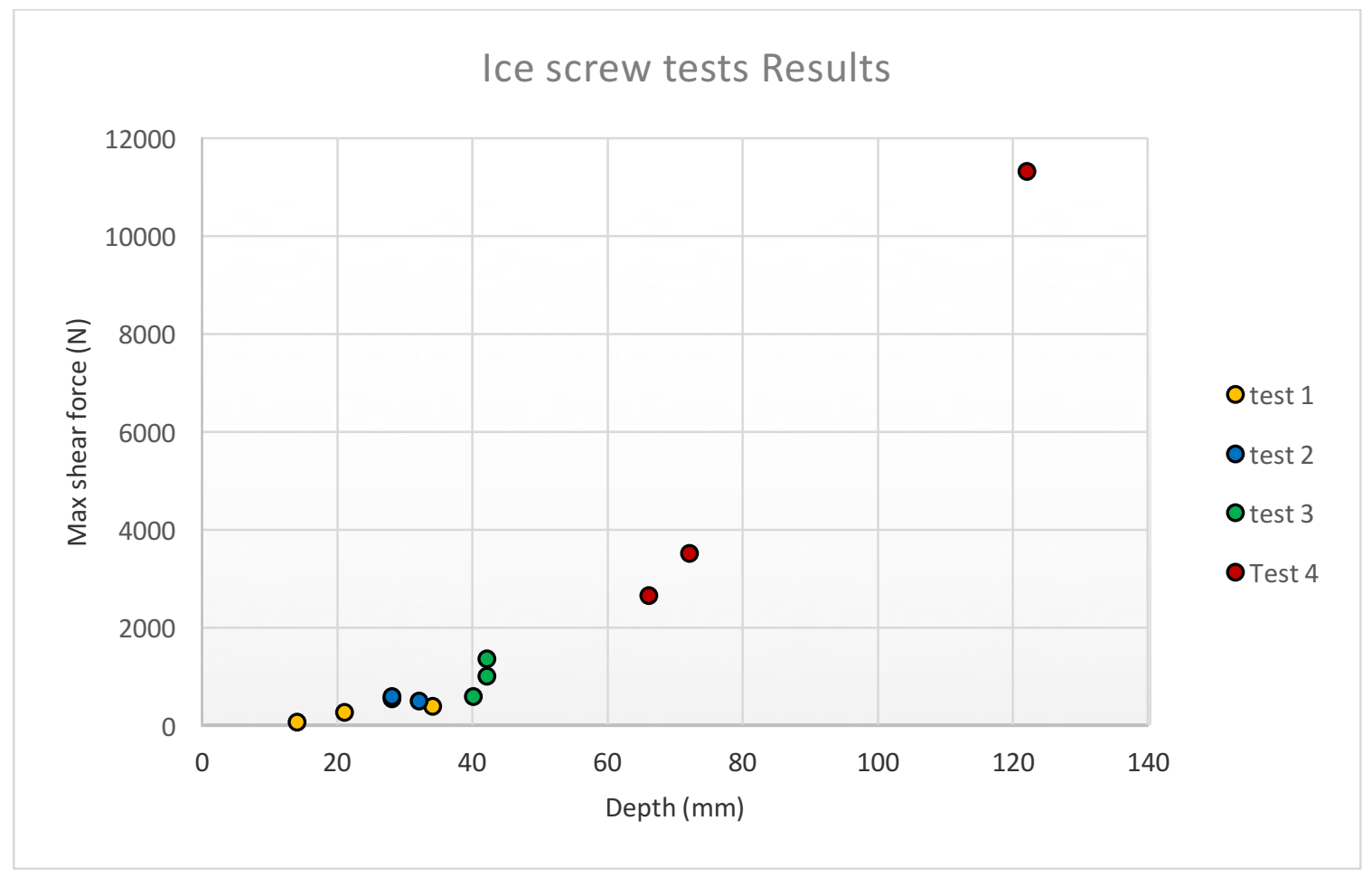

Figure 54 Ice Screw Shear Strength results

The ice screw tests seem to suggest a nonlinear relationship between shear forces and depth, although the small number of data doesn't allow to say it for certain. It is clear, however, that the $10 \mathrm{KN}$ force is hardly reached for length below $100 \mathrm{~mm}$ when the load is applied as pure shear. We expected this result, since the screw is optimized to work using the thread rather than acting as a fixed beam.

Another concern is related to the creep process happening in the ice. The mechanical proprieties of ice change greatly with the temperature and the creep phenomena become more and more relevant as it gets closer to the melting temperature of $0^{\circ} \mathrm{C}$. The process is clearly visible in Table 4 where it's clear, how during the compression machine pauses, the ice greatly relaxes the acting forces. The Table 4 refer to the last performed test. 


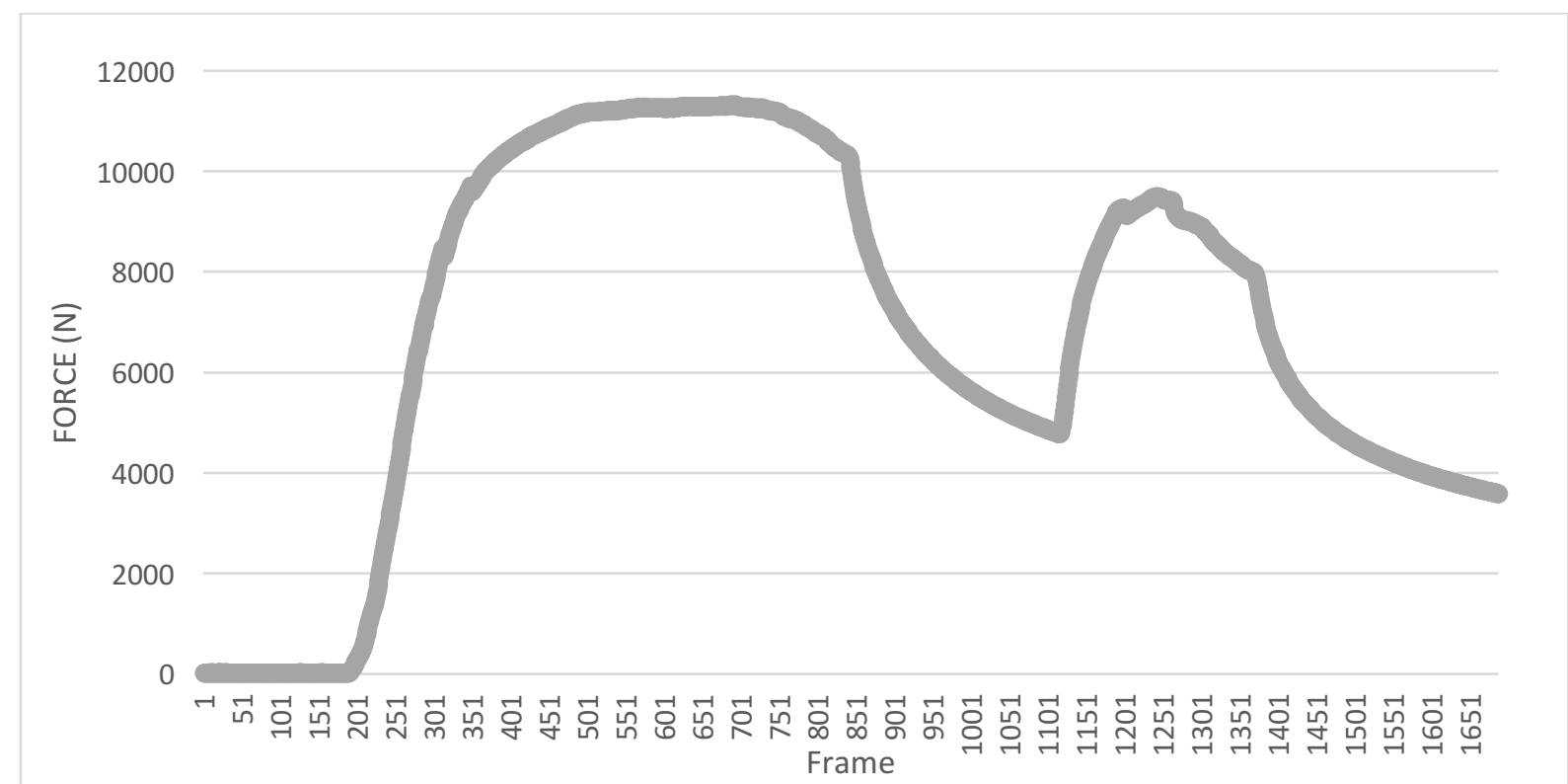

Table 4 Ice screw test result plot

\subsubsection{Comparison with finite element model (FEM)}

The experiments gave us a good view of the ice screws behavior, yet it is still unknown how the ice screw properties scale with the diameter. A proper test requires having the same screw geometry, scaled down and tested in the same condition. That was not possible to do since the producing company offered only a single size and to have it properly manufactured was not trivial.

To have a first order approximation however, it was possible to simulate the problem with a finite element model (FEM) analysis. Clearly such procedure would be too complex or too unreliable for obtaining absolute values, given the complexity of the problem, but it would suffice to compare the results between different diameter. The models were prepared with diameter 10, 7.5 and $5 \mathrm{~mm}$. The load, $1000 \mathrm{~N}$, is applied as uniform shear load at a $5 \mathrm{~mm}$ distance from the ice surface. The screw is fully modeled and penetrates the ice for a depth of $40 \mathrm{~mm}$ regardless of the diameter. The contact regions are limited to the thread surfaces and are modeled as bonded. The simulations are under linear elastic assumption and the Young module chosen for the ice and screw material are $3 \mathrm{GPa}$ and $206 \mathrm{GPa}$ respectively. Supposing the ice fracture to be the critical condition, we ignored the screw condition. For each simulation, we extracted the minimum equivalent stress present in a full thread in the ice.

\begin{tabular}{l|l} 
DIAMETER (MM) & ICE MPA \\
\hline $\mathbf{1 0}$ & 3.84 \\
$\mathbf{7 . 5}$ & 6.5 \\
$\mathbf{5}$ & 12.5
\end{tabular}

Given the results, it seems that the relation between diameter and ultimate strength follows a square proportion. An experimental study should however be performed to verify and refine such hypothesis. 


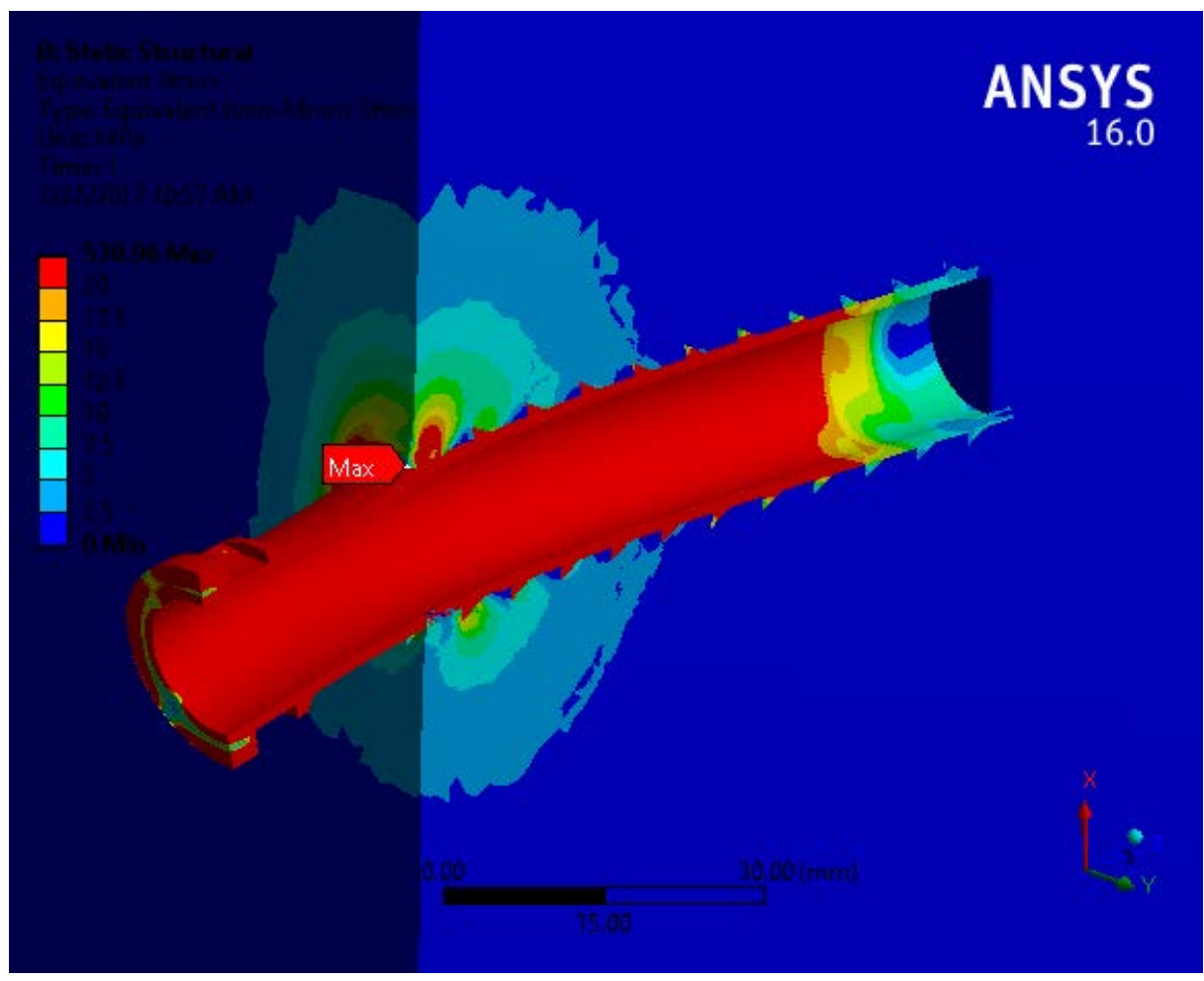

Figure 55 FEM analysis. Test example. 


\section{Mechanical prototype design}

We developed a prototype design for two reasons. One is to serve as a reference point for the estimation of DM's power requirement in the system trade study in Section 4.2. The other reason is to validate that the mechanical system can be miniaturized to the size required by EVE. For this purpose, our prototype design only uses existing technologies/materials and commercially available mechanical parts (e.g., motors, gear boxes), which are included in our CAD design. In addition, to verify the design an 3D printed model has been created, which is not actuated but all the joints are movable.

\subsection{Considered Configurations}

In this section, a few configurations of the DM will be proposed, analyzed, and compared. As we mentioned previously, the resulting prototype design is a rather conventional limbed robot with three body sections. However, before converging to this design, various other innovative designs were considered, which could still be viable design. Therefore we will describe those alternative designs in this section, too.

\subsubsection{Linear actuation vs joints}

The first choice regarding the mobility system of the $\mathrm{DM}$ is related with the main modus of advancement. Two main architecture are available.

The first architecture is a joint actuated limb structure, that is conceptually the typical biological solution for animals, human included. The advancement is achieved through the arms being able to relocate the anchoring system thanks to a chain of elbow's joints in the same way a human arm works. A similar platform working on that concept is LEMUR (Parness, et al., 2012). Such a system allows a high degree of freedom and adaptability.

The second architecture decompose the advancement actuation and angle adjustment, so that the load is only carried by a single primary linear actuator, while the secondary actuators never work against the flow directly. Furthermore, it doesn't require changing its frontal section area in any phase

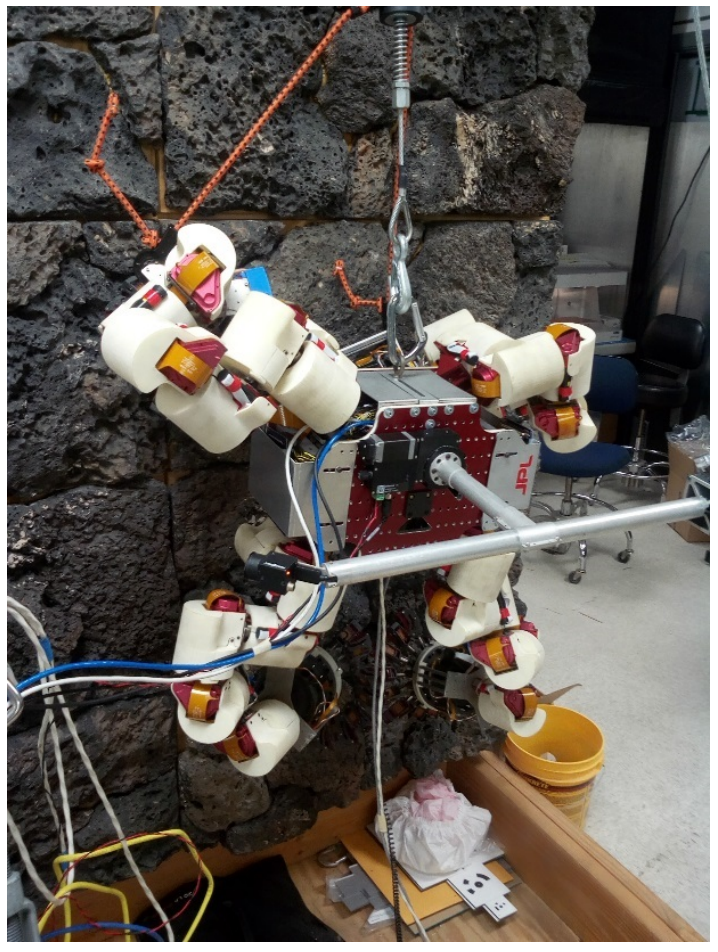

Figure 56 LEMUR climber. JPL 347 Robotic Section of the advancement and so be able to use most of its capability even in the smallest vent section for which the DM is designed.

\subsubsection{Worm structure}

On the opposite of a joint actuated robot stands the worm shaped DM. It offers the highest reliability and performance in term of resisting the flow sacrificing some flexibility to unexpected 
situations. The design is mainly composed by 2 anchoring sections, each one possessing two embedded ice screw. Additionally, it has a pushing pin capable of pushing the ice and balance the aerodynamic moment on the body. In between those two modules a third main module is connected through 2 rigid and actuated joints. Those joint must allow at least two degree of freedom. The third main module act as a linear actuator, allowing the DM to increase and decrease his own length while connecting or disconnecting the front and the back screw.

A single step movement from contracted position is composed by two phases: First the frontal screws dethatch them self, the linear actuator extends and the two screws newly attach themselves on the icy wall. Secondly, the back screws dethatch, the linear actuator contracts and the screws attach as well.

The cycle is then repeated for each step, and the length of the step is equal to length extension of the middle linear actuator. In this configuration, a single linear actuator must take all the drag load.

The advantages are:

- The capability of having a very compact design, easy to manipulate to obtain certain aerodynamic proprieties such as low drag and negative lift.

- Limited number of actuators, and as such limited failure points.

- The ice screw can easily act as sampler and the taken sample are easy to manipulate.

- The main actuator is completely isolated from the other degrees of freedom and preserve most of his capabilities even in case of other joint failure

The main disadvantages are the limited adaptability to unexpected feature as well as the management of the aerodynamic moment acting on the body while only the back crews are attached.

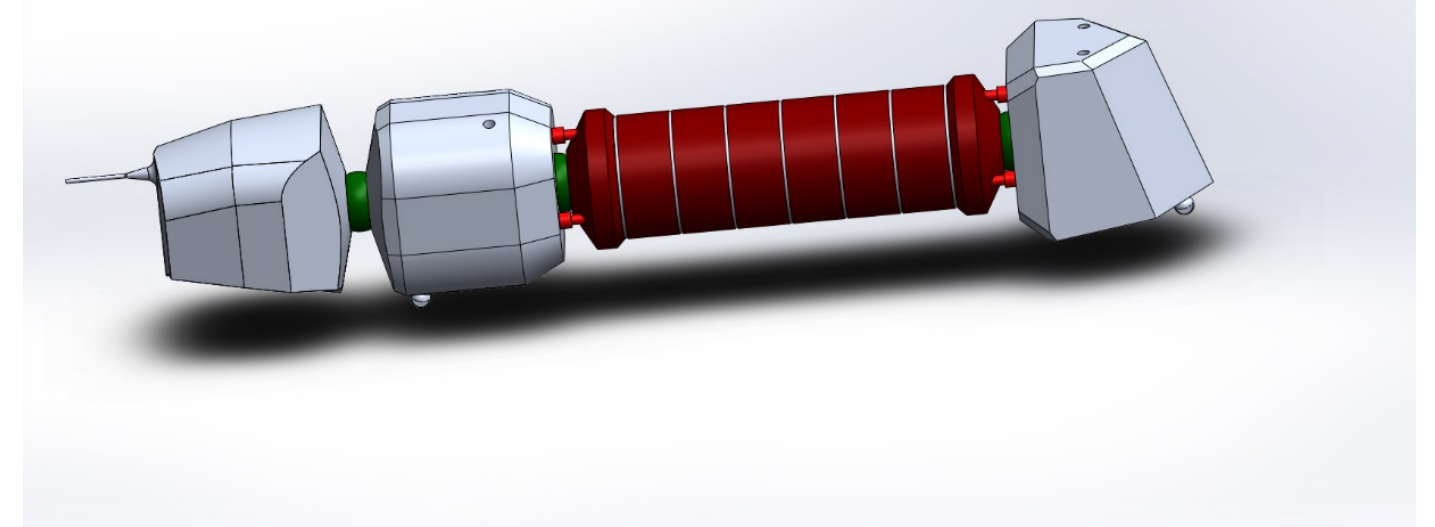

Figure 57 Worm Design. Conceptual model 


\subsubsection{Four limbs head}

A four limbs head concept uses a main module to store the mobility system. The mobility system is composing of four limbs each of which has four rotational actuate degree of freedom and one linear actuator for a total of five degree of freedom per limb. A jointed section body follow the main module. While those joint gains more adaptability in being actuated, those extra degrees of freedom are way less critical that in the other cases and only facilitate the adaptability of the climber to the vent shape.

Three major limitations are connected to this design:

- The limited space for the mobility system compromises the maximus torque those joint can generate or even renders the realization unfeasible.

- The two back legs maximum extension is limited by the body shape when constrained in a small section vent

- The inversion of movement for return to the surface is harder to acquire.

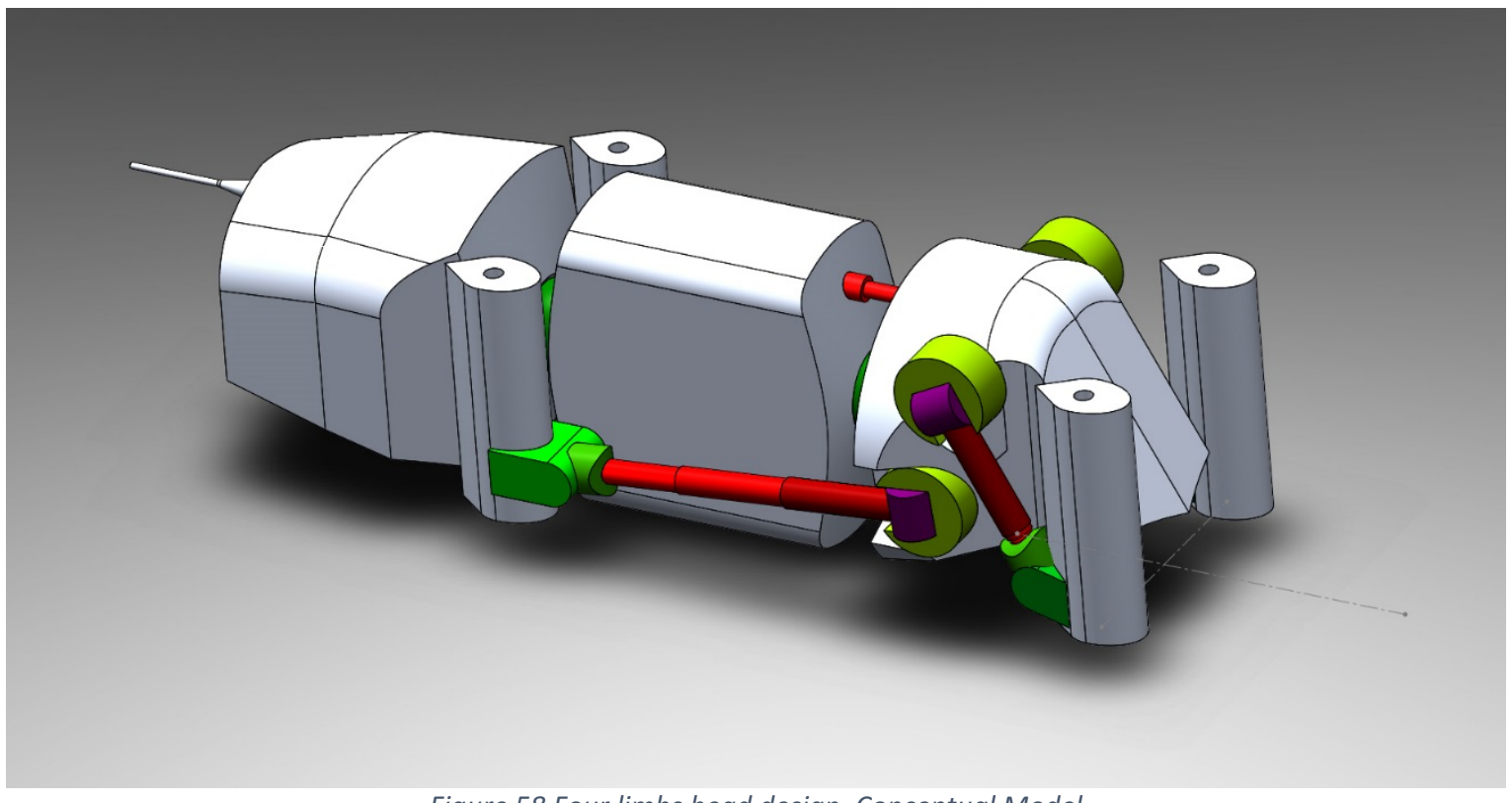

Figure 58 Four limbs head design. Conceptual Model

\subsubsection{Two limbs}

A variant of a four limbs design is represented by a two-limb design, allowing the kinematic structure for the arms to have enough room to be realized. The idea combines the two previous designs, combining a two arm and embedded screws as well as pushing pins to react the moments. The design however still holds the problem to be hard to reverse. In addition, the pushing pins still hold a degree of uncertainty since their efficacy need to be proved. The step is divided in three phases: One for each arm to anchor, and one for the main body to advance and anchor. 


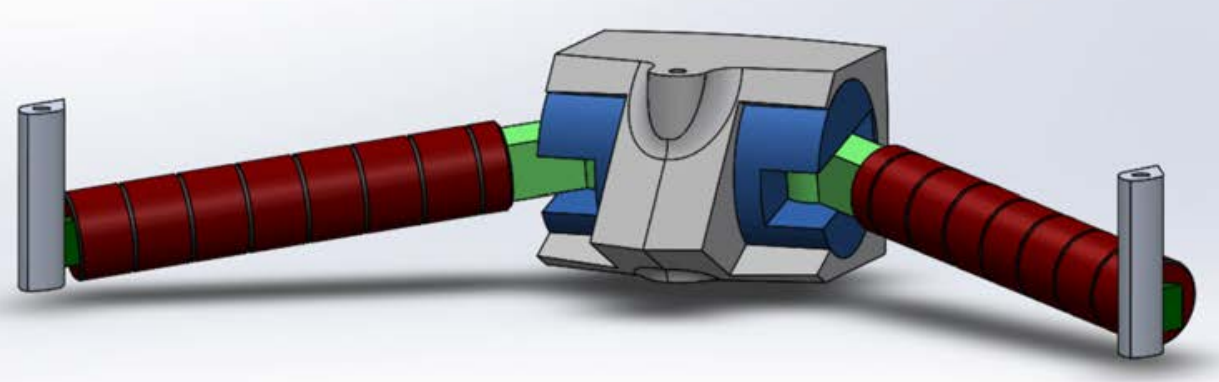

\subsubsection{Two plus two limbs}

The last and final solution took in account all the advantages and disadvantages of the previous design. It has been chosen not to use pushing pins, and a four-limbed robot design was preferred. However, the previous four-limbed design has issues, especially the volume allocation was too prohibitive. The design uses two sections with two limbs each, and a middle section for accommodating instruments, connected by rigid actuated joints. The working principle is the same of the four-limbed one, but it solves the volume problem and grant the capability to easily invert direction. The model will be further analyzed. The gait of this design is shown in Figure 59.

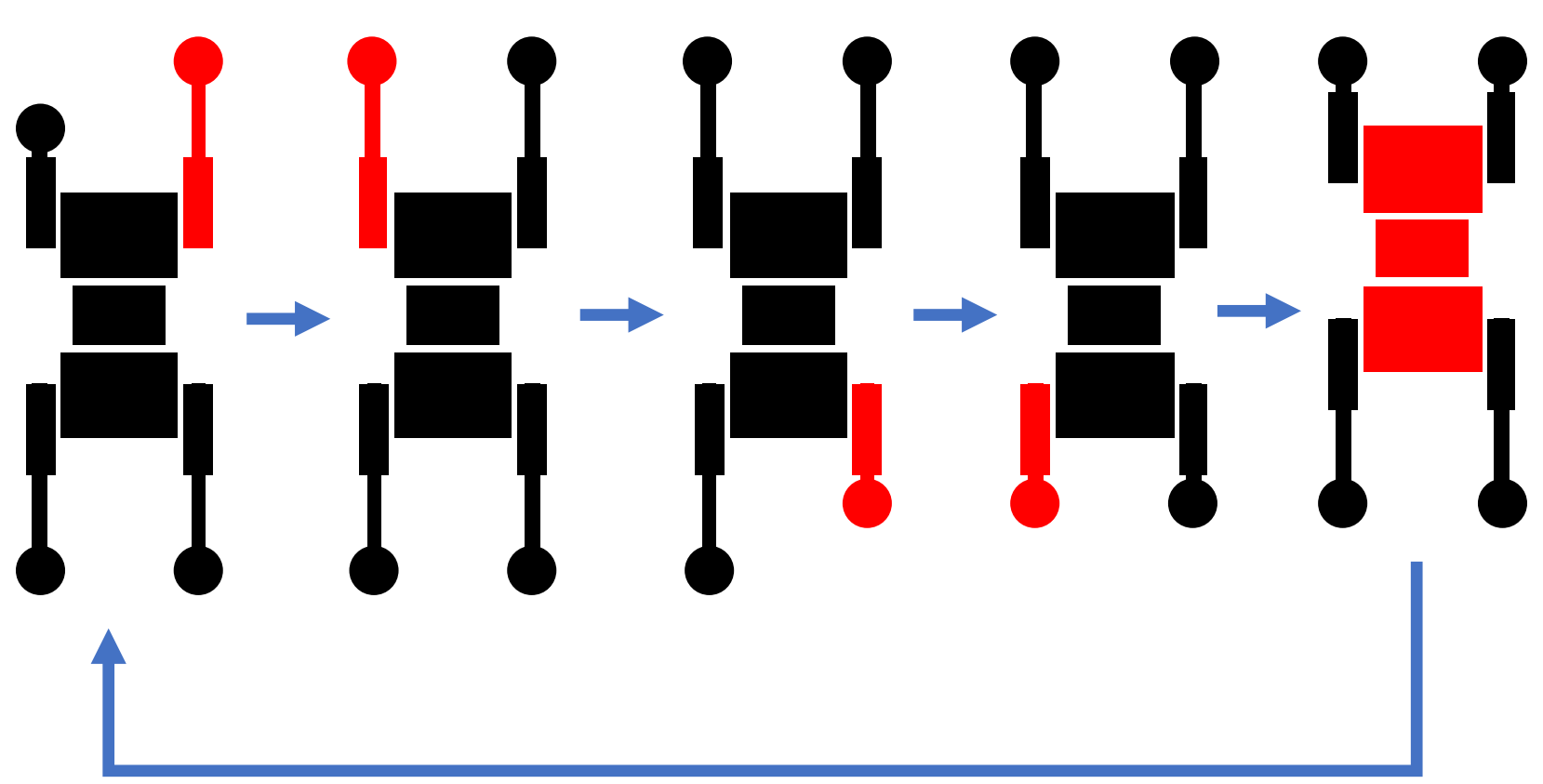

Figure 59 DM's gait 


\subsection{Linear actuators}

As the general architecture of the DM was selected, the first detail to analyses was the linear actuator: in fact, the design relays on the linear actuator to shoulder the aerodynamic load independently from the other degree of freedom. The first phase was to analyze the different solution, highlight pros and cons, then, once selected a winning solution, estimate the characteristics and lay a possible design. As a general goal, it has been fixed a $1000 \mathrm{~N}$ load in both extension and compression need to be achieved for any given condition. The requirement has been decided after a first estimate of the aerodynamic forces.

\subsubsection{Actuator selection}

The considered linear actuators are:

1) Hydraulic and pneumatic actuator

2) EMA Electro-mechanical actuator

a) Screw

b) Segmented spindle [Kataka]

c) Rigid belt actuator

d) Helical band actuator [Spiralift]

3) Amplified piezoelectric actuator

4) SMA actuators [Large forces shape memory alloy actuator]

5) Electromagnetic actuator [Moving coil]

6) Boom Deployer

We will now analyze some of the most interesting solution:

- Hydraulic actuator: Hydraulic actuator offers the highest force for unit of volume, but present several major issues linked to the need of a pump and hydraulic logic as well as the problem related with hydraulic liquid exposed to cryogenic temperature and vacuum during the flight. Hydraulic actuators are generally not used for deep space missions, but they are worth considering for future missions.

- Piezoelectric actuator: With attention for amplified piezoelectric, those type of actuator offers the advantage to use little to no lubricants and are generally unaffected from vacuum and cold exposure, making them ideal for space applications. Piezoelectric actuator come in great variety and design, however our first research could not find any commercial product similar to what required.

- Electro-mechanical actuators (EMA): Under the category of Electro-mechanical actuators falls the linear screw actuator, that is the most common linear actuator, as well as some more exotic solution like a segmented spindle of a rigid belt actuator. Mostly influenced by extreme environments, they fit well for both high forces, precise control and small dimension. The major concern is to grant the correct functioning and resistance to damage under irregular and sudden loads and solicitations. 


\subsubsection{Final choice}

Considering the scope of this study is a feasibility analysis, it is preferable to prove that a traditional design, easier to verify and design, can be used for the goal. After a few preliminary studies, we decided to develop a customized screw linear actuator. Of course, when it will become necessary to optimize the performances, it may be interesting to reevaluate the choice and design of the linear actuator.

\subsubsection{Actuator details and verification}

A first order analysis was performed to define the major characteristic required from a screw actuator. For deciding sizes, we referred to the Nook products and verification ${ }^{11}$, while we took as reference for motors and gearboxes the products of the Maxon.

For the linear actuator, considering the formula:

Where:

$$
T=\frac{F \cdot p h}{2 \pi \mu}
$$

$T$ is the gearbox torque

$F$ is the actuator output $(1000 \mathrm{~N})$

$p h$ is the pitch of the screw

$\mu$ is the efficiency of the linear actuator ( 0.4 or 0.8 if a ball recycling system is present or not)

We analyzed a $12 \mathrm{~mm}$ diameter screw, single starts, couple with a Maxon gearbox $10 \mathrm{~mm}$ diameter with a $0.15 \mathrm{Nm}$ max. output torque. It should be considered that the only type of gearbox available for that motor diameter, unless for customized orders, are with plastic gears, that obviously have lower performances compared to the nowadays existing metal and ceramic gearboxes.

\begin{tabular}{l|lll}
\multicolumn{1}{l}{ SCREWS } & PITCH (MM) & TORQUE REQUIRED & FEASIBILITY \\
\hline$\varnothing 12$ WITHOUT BALLS & 2 & 0,79 & \\
$\varnothing 12$ WITH BALLS & 2 & 0,39 & need higher quality gearbox \\
$\varnothing 12$ WITHOUT BALLS & 5 & 1,98 & \\
$\varnothing 12$ WITH BALLS & 5 & 0,99 & extremely strong gearbox \\
$\varnothing 12$ WITHOUT BALLS & 10 & 3,97 & need higher quality gearbox \\
$\varnothing 12$ WITH BALLS & 10 & 1,98 &
\end{tabular}

We want now to verify the Linear actuator itself to be able to withstand the expected operative life. We impose a $98 \%$ reliability, $1000 \mathrm{~N}$ to extract and $300 \mathrm{~N}$ to retract and a life reduction factor between 2.5 and 3.5 as specified for irregular loads and vibrations ${ }^{12}$. We then calculated the mean load ${ }^{13}$ and then defined the travelable distance.

$$
F_{a v}=\sqrt[3]{\sum_{i} F_{i}^{3} \cdot \frac{n_{i}}{N}}
$$

\footnotetext{
${ }^{11}$ http://www.nookindustries.com/

${ }^{12}$ We used the formula available at http://www.nookindustries.com/

13 http://www.nookindustries.com/
} 
$\frac{n_{i}}{N}$ is the fraction of cycles.

$$
D(k m)=10^{3} \cdot \mu_{98 \%} \cdot\left(\frac{C_{s}}{F_{a v} \cdot f_{w}}\right)
$$

$C_{S}$ is an empirical coefficient related to the screw ${ }^{14}$.

$f_{w}$ is the life reduction factor.

$\mu_{98 \%}$ is the reliability factor $(0.44)$

SCREWS
$\varnothing 12$ MIN LIFE
$\varnothing 12$ MAX LIFE
$\varnothing 12$ MIN LIFE
$\varnothing 12$ MAX LIFE
$\varnothing 12$ MIN LIFE
$\varnothing 12$ MAX LIFE

PITCH (MM)

$\mathrm{C}_{\mathrm{S}}$

KM OF TRADABLE ROAD

\begin{tabular}{|lll}
2 & 2820 & 0.54 \\
2 & 2820 & 1.50 \\
5 & 6850 & 19.6 \\
5 & 6850 & 53.8 \\
10 & 7050 & 42.7 \\
10 & 7050 & 117
\end{tabular}

The last thing to verify is the movement speed that is directly related to the motor. Referring again to the Maxon motors, we selected 2 motor type. For each motor, we consider the case with and without recycling balls. We consider a typical gearbox efficiency of 0.8 . We calculate then the average advancement speed.

\begin{tabular}{l|lll} 
MOTOR & WATT & OUTPUT WATTS & SPEED M/S \\
\hline BRUSHLESS, WITH BALLS & 8 & 5.12 & 0.00512 \\
BRUSHLESS, WITHOUT BALLS & 8 & 2.56 & 0.00256 \\
$\begin{array}{l}\text { PRECISION METAL BRUSHED, } \\
\text { WITH BALLS }\end{array}$ & 1,5 & 0.96 & 0.00096 \\
$\begin{array}{l}\text { PRECISION METAL BRUSHED, } \\
\text { WITHOUT BALLS }\end{array}$ & 1,5 & 0.48 & 0.00048
\end{tabular}

Considering the four-limed design, the overall speed will be the 5 time less than the single actuator, as each leg must move independently, and then once the four screws are relocated, the main body moves forward pushed by all the four actuators, as illustrated in Figure 59.

As a proof design, it has been chosen a $2 \mathrm{~mm}$ pitch with recycling balls. To reduce the required volume, we propose a new potential design, where we use a threated tube in which a small screw section is actuated by an electric motor, caged as well inside the tube, allowing to have a much more compact design compared to the traditional architecture.

\footnotetext{
${ }^{14}$ http://www.questek.com/ferrium-c64.html
} 


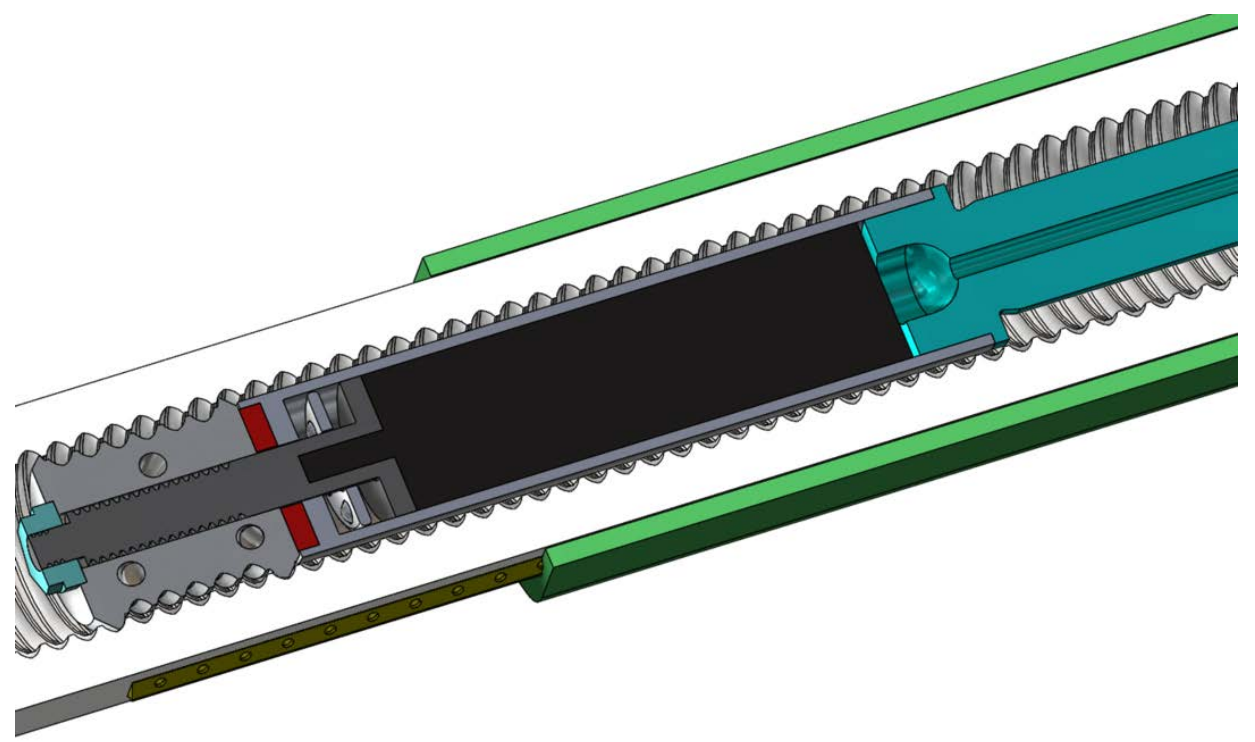

Figure 60 Design details: section linear actuator.

\subsection{DM kinematics}

Once defined the linear actuator geometry, the remaining 4 degree of freedom must be actuated. Since they don't work directly against the load, it becomes hard to quantify the torque requirement for each joint. Instead it has been decided to try to maximize the torque producible using the available space at best, and then extract the best performances available. Our analysis concluded that a minimum of 5 degree of freedom are required for each arm to allow the DM to adapt to the entire range of expectable geometry, so to allow to choose the angulation of the screw ( 2 DoF) and the location of the screw compared to the main body (linear actuator plus 2 DoF)

\subsubsection{Degree of freedom and design}

The screw extension must have two degree of freedom, torsion along the arm and rotation perpendicular to the plane defined by the arm and screw axes. At the base of the arms instead it's required an actuated shoulder joint with two degree of freedom. Of those only the torsional degree of freedom can be both allocated inside the main body or at the extreme of the arm. For the design proposed we will propose the latter. The major problem is to fit the two degree of freedom of the shoulder in the target volume. To do so it has been decided to decouple the two systems using a cables system to actuate on of the degree of freedom. That requires two wires for each joint that need to be actuated independently, but allows to allocate freely the involved actuators. 


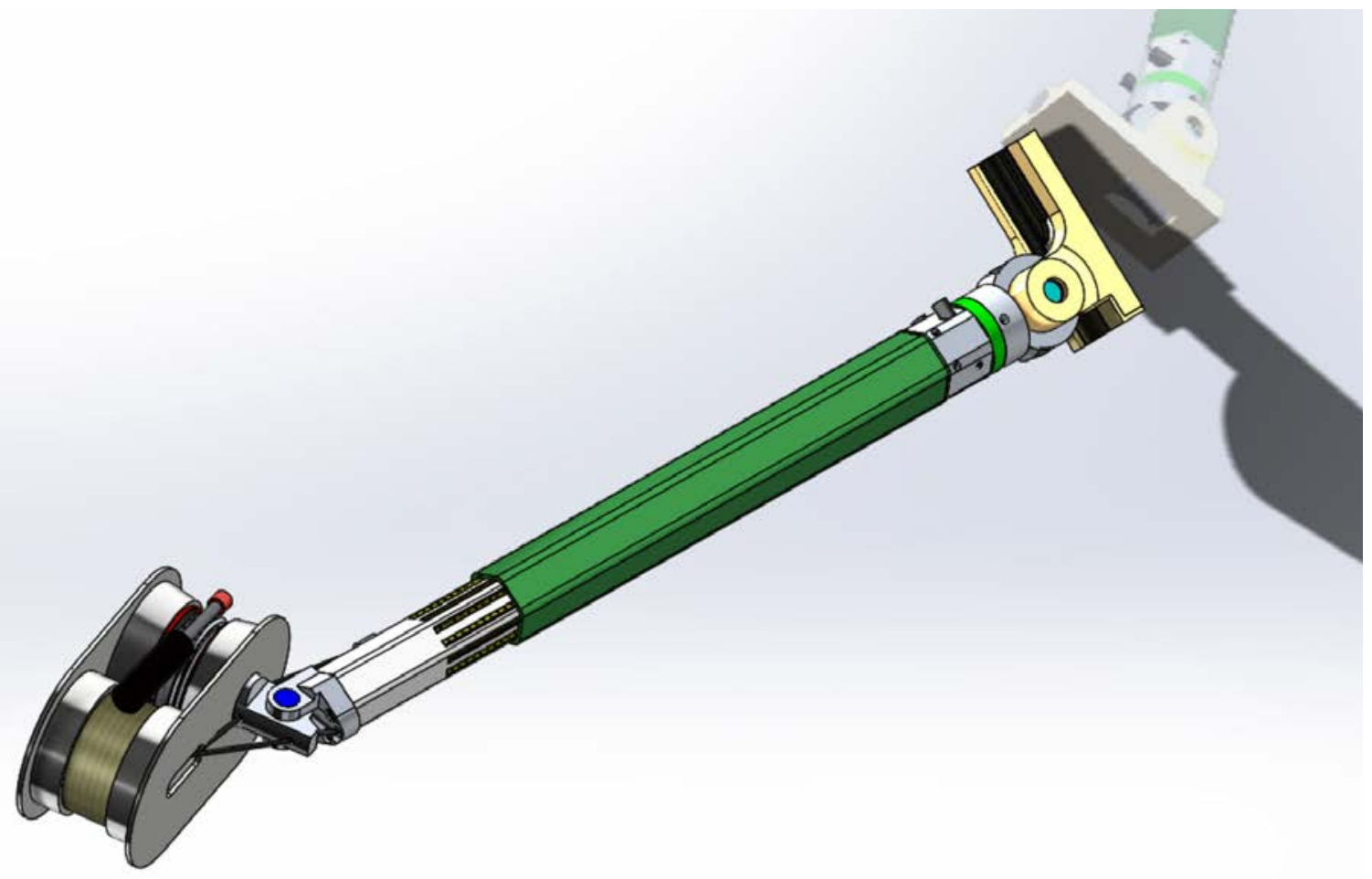

Figure 61 Design: single arm mechanical architecture.

Such design allows to be mounted interchanging the two degree of freedom, allowing two different configurations. The configuration chosen uses the cables for horizontal movements, while a direct gear transmission feeds the vertical movements and grants the drilling starting pressure to the screw. Starting from such design we then elaborated a possible shape of what the DM head may look like, keeping in account the actual locomotion shape and size. 


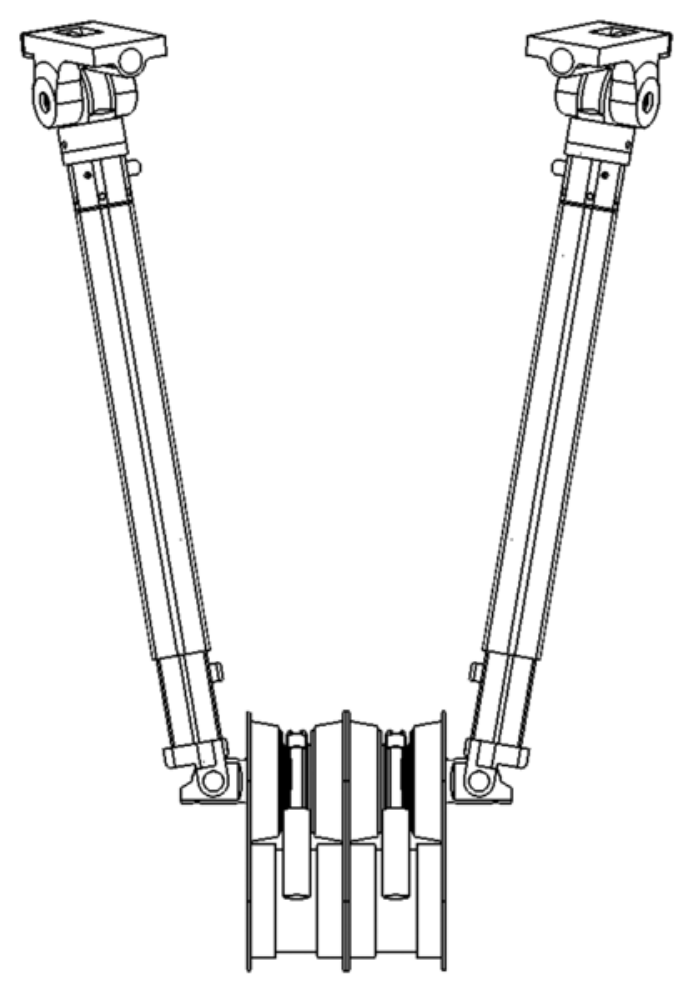

Figure 62 Design: Full mechanical part design. Choose option.

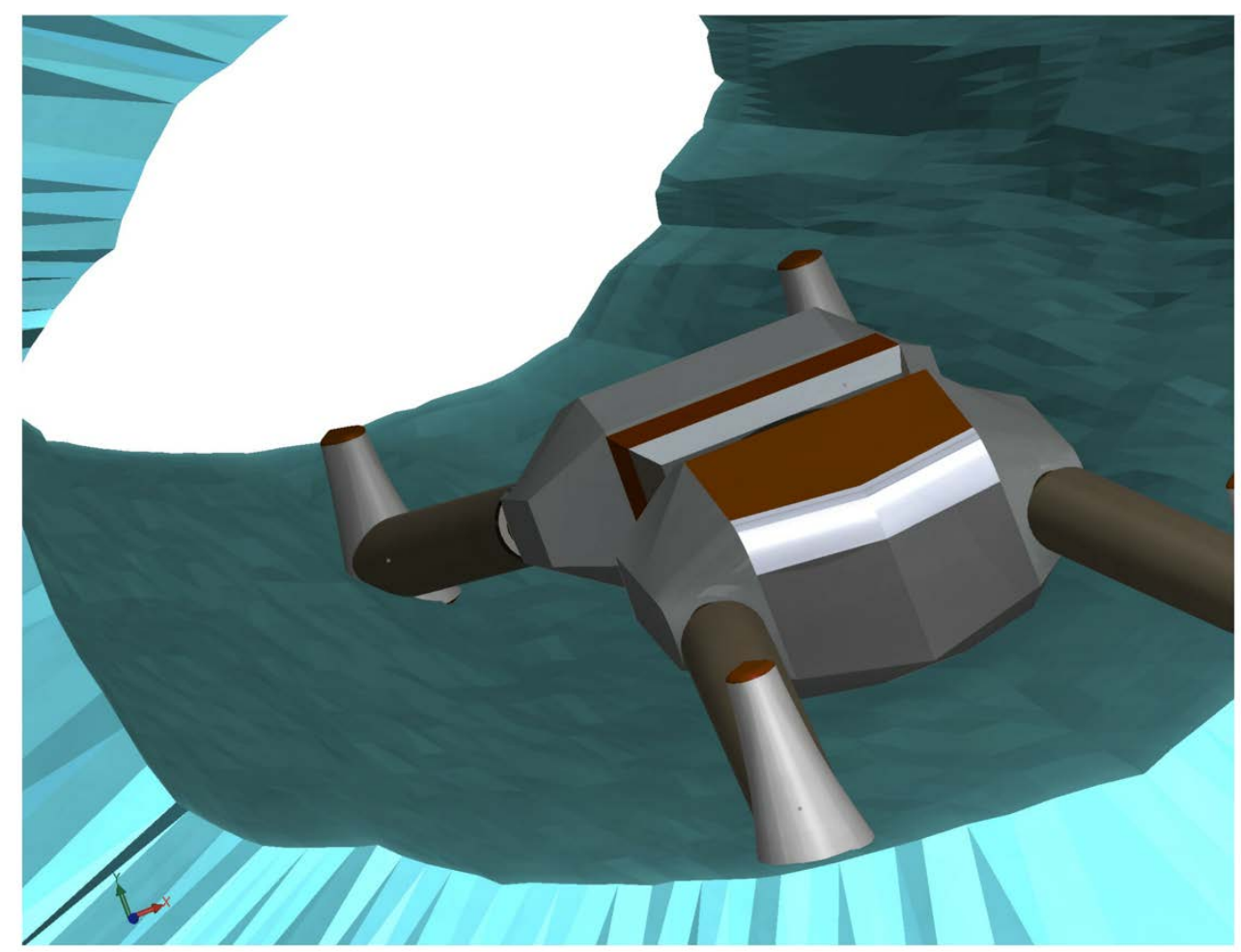

Figure 63 Design: Outfit concept 


\subsubsection{Section joints}

We will now analyze in more detail the solution proposed and the verification generated. We start with the head of the arm, where the connection with the screw system is located. The screw mechanism is never represented, and as a study choice, never been modeled since a very similar concept is still in development for a project related to LEMUR.

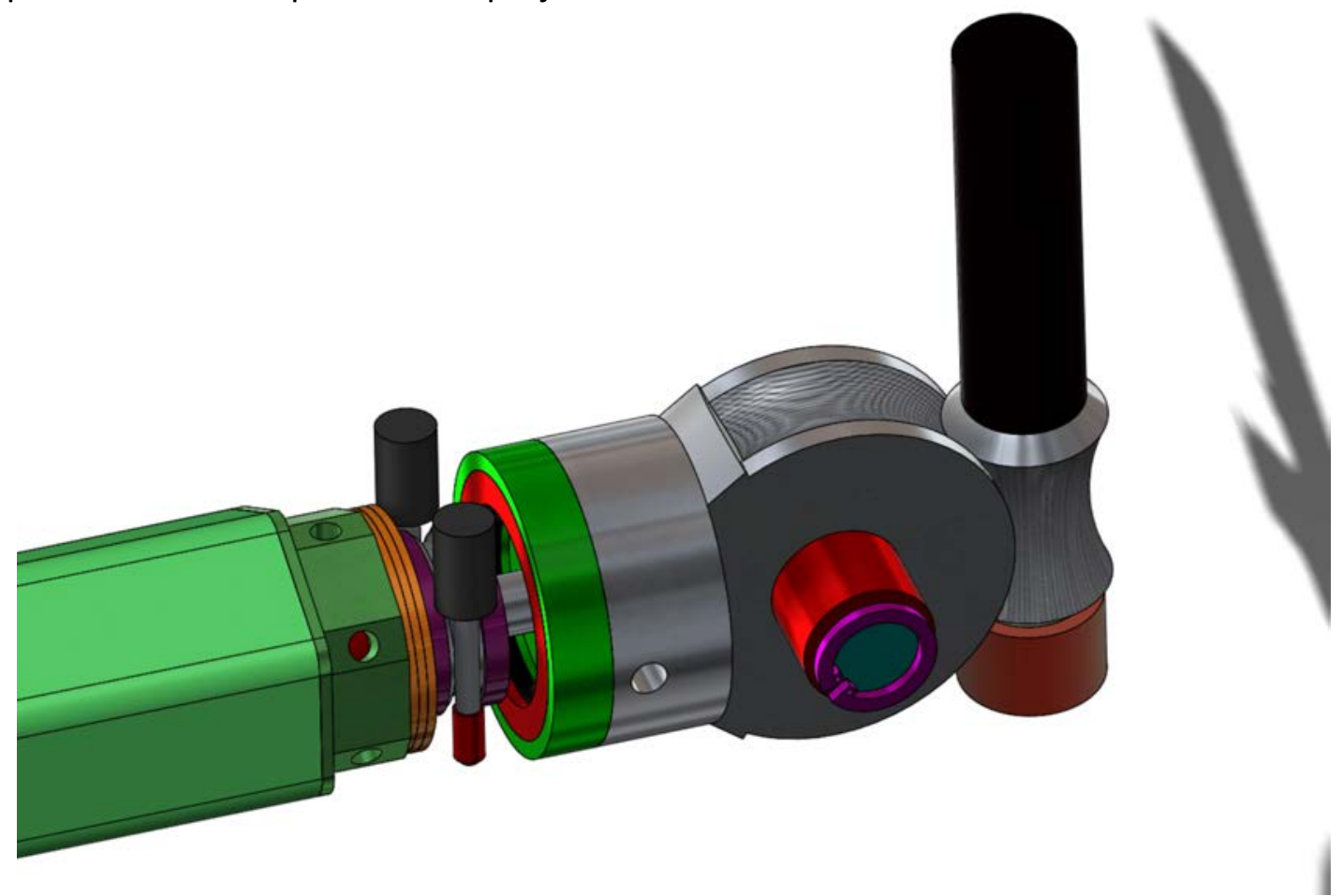

Figure 64 Design: Upper arm details.

Figure 64 illustrates a couple of hyperbolic worm gear [gear 1] and coupled half round gear to control the vertical angle of the screw. The motor used has a $10 \mathrm{~mm}$ diameter as well. A couple of needle bearings (SKF K8x11x8 TN as reference) transmit the load from the screw support to the arm. In the figure are also visible a couple of motors, $5 \mathrm{~mm}$ diameter, connected to a couple of worm gears [gear 2]. This is the mechanism reasonable for the rotation on the arm axis. All the verification will be conducted considering a single motor, the drawing just shows that, in case of need, it's possible to double the torque output. 


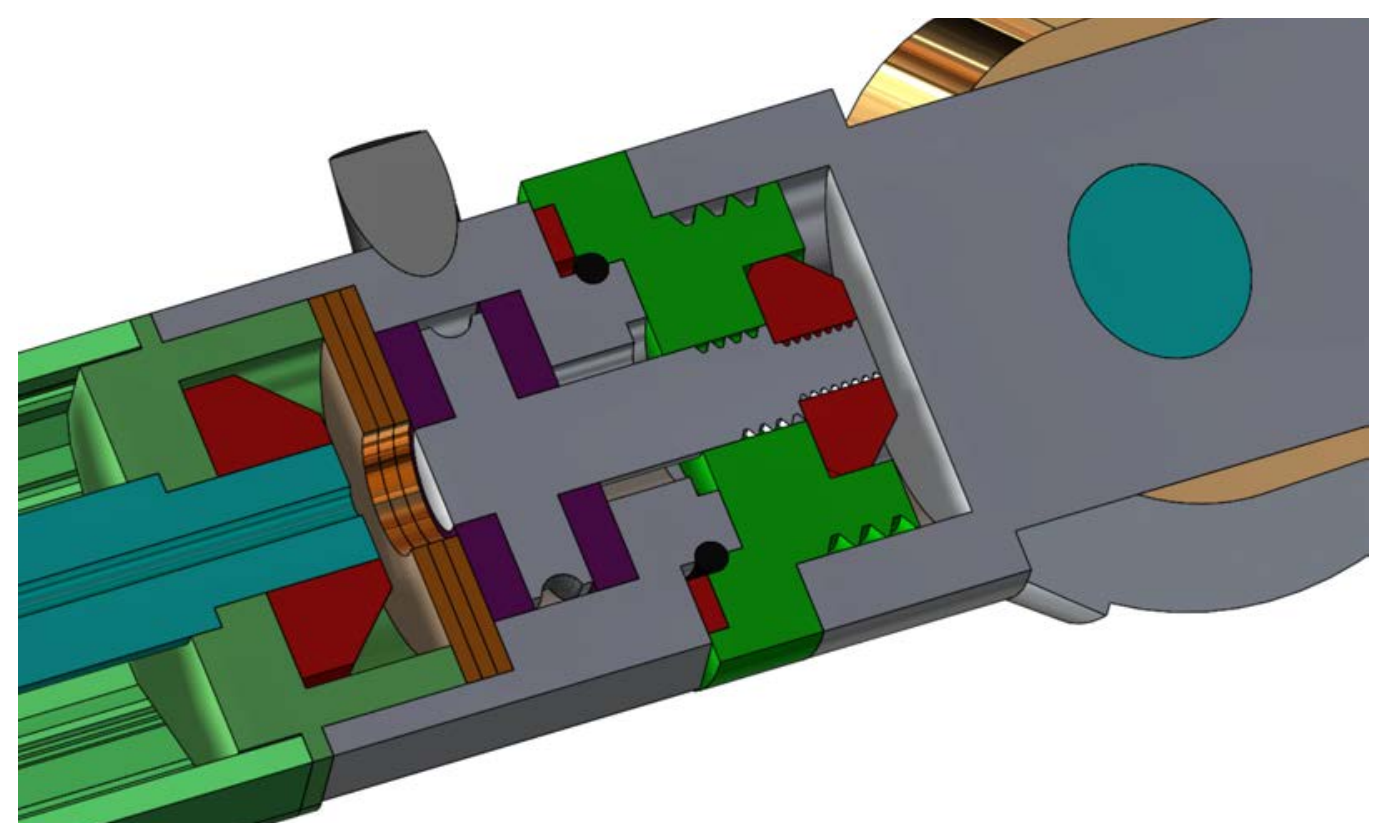

Figure 65 Design: Upper arm, section.

At the base of the arm the other two rotations are acquired respectively by a worm gear [gear 3] couple and a tendons system. The tendons actuator is yet to be designed in detail, but the two wires need to be actuated independently. A couple of roller angular contact bearings hold the rotating shaft, while another needle bearing connect the arm to the shaft. Considering two arms the design can be further compacted using a single central bearing.

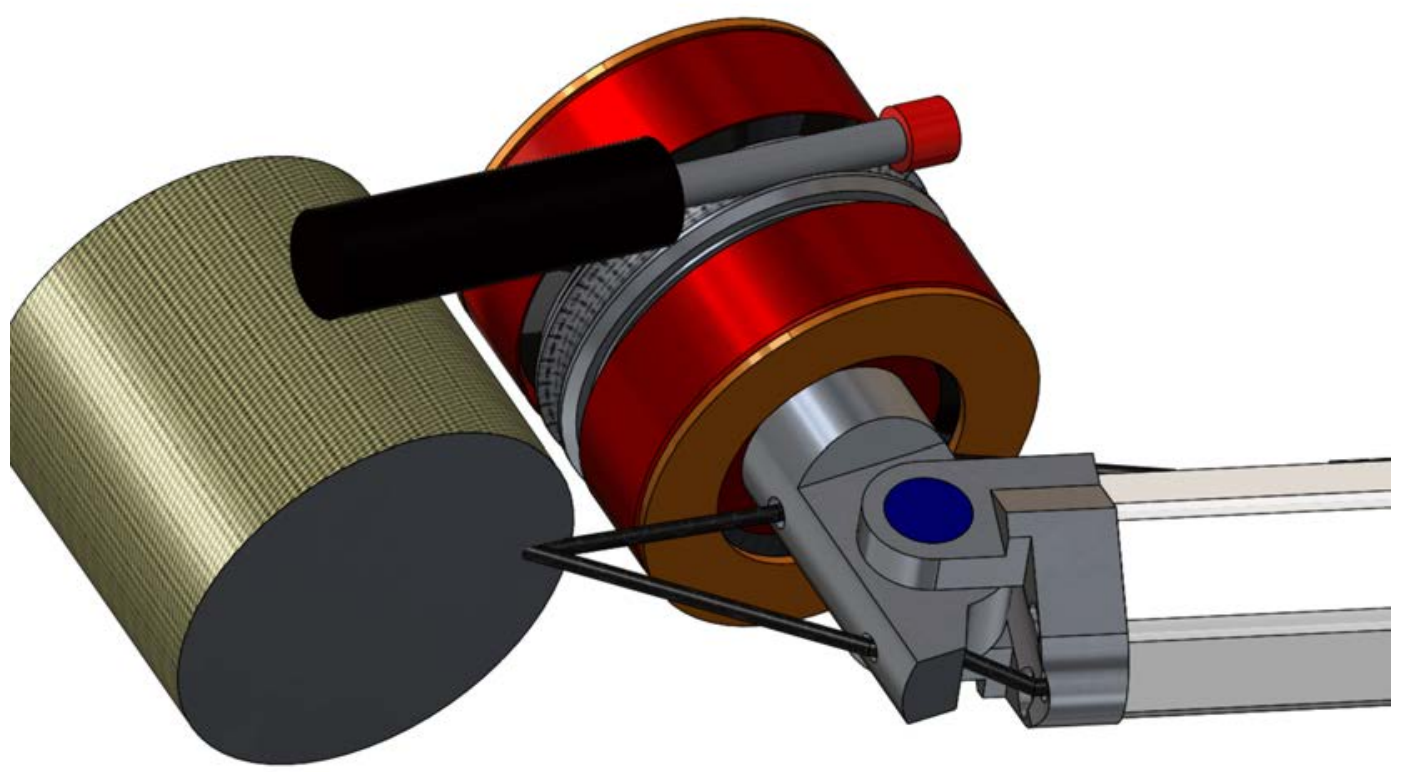

Figure 66 Design: DM internal actuations. 


\subsubsection{Arms joints and verification}

Using the same procedure for the linear actuator, a set of critical components have been analyzed to verify the feasibility of the given design. Follows then the analysis of the chosen components and the criteria adopted.

\subsubsection{Ropes}

The ropes components are responsible to transmit the torque from the actuator to the arm. We assume a range of reasonable diameter for the rope and we then calculate the maximum torque that can be obtained and the torque that needs to be applied by the actuators to the pulley. Approximatively the conditions are equal for both the ropes. It is possible, if necessary, to add an internal pullet to half the required torque from the actuator. It will obviously reduce the efficiency, but we leave such analysis for future developments.

We make the following assumptions:

\begin{tabular}{|c|c|c|c|}
\hline \multicolumn{3}{|l|}{ Ultimate Stress } & $1000 \mathrm{MPa}$ \\
\hline Safety factor & & & 1.2 \\
\hline Pulley diameter & & & $40 \mathrm{~mm}$ \\
\hline \multicolumn{4}{|c|}{ From such we obtain the following results } \\
\hline ROPE DIAMETER (MM) & AREA $\left(M^{2}\right)$ & MAX TORQUE (NM) & $\begin{array}{l}\text { TORQUE ON THE PULLEY } \\
\text { (NM) }\end{array}$ \\
\hline 0,8 & 0,50 & 4,60 & 8,37 \\
\hline 0,9 & 0,64 & 5,83 & 10,6 \\
\hline 1 & 0,79 & 7,19 & 13,0 \\
\hline 1,1 & 0,95 & 8,71 & 15,8 \\
\hline 1,2 & 1,13 & 10,3 & 18,8 \\
\hline 1,3 & 1,33 & 12,1 & 22,1 \\
\hline
\end{tabular}

\subsubsection{Motor cage, linear actuator}

The component that encages the motor is the only newly designed component which has been verified at the phase. The reason is that it is the component taking supporting the highest structural stress by far. So as long as this component doesn't present any issues, we can assume that any other static component is not critical for the design.

For the material proprieties, we take as reference a Titanium Ti-5Al-2.5Sn, ELI, Annealed Alloy and reduce its ultimate strength by a factor of 0.7 to take account of space deterioration. That's it's necessary since space rated materials proprieties are considered sensible information and were not available to consult. As such we assumed a Yield Strength equal $581 \mathrm{MPa}$, A Fatigue strength at $3 \cdot 10^{6}$ cycles equal $434 \mathrm{MPa}$ and a Young Modulus equal $100 \mathrm{GPa}$.

Taking dimensions' reference from the preliminary design, results are the following:

Radius, external 
$I_{\mathbf{x}}$

Compression load, Euler instability Ks=2

21436

Traction load Kt=3

10992

Table 6 Motor cage verification

\subsubsection{Bearings}

Follows an estimate of the bearing loads and verification for static load. SKF standard bearings ${ }^{15}$ are used as reference for both dimensions and loads. For future analysis or developments two factors are to be taken in consideration: Bearing proprieties deteriorate in space and cold environment due to the need of using specific lubricant and sealings. The listed proprieties are referred to traditional bearings, which proprieties data are downgraded to fit reliability standards for given number of cycles; for this specific application, nowadays technologies allow much better performance.

\begin{tabular}{l|lllll} 
BEARING NAME & SKF K8X11X8 & SKF AXK 0515 & SKF BA 4 & $\begin{array}{l}\text { SKF K8X11X8 } \\
\text { TN }\end{array}$ & SKF 30203 J2 \\
\hline LOCATION & $\begin{array}{l}\text { Toupled to } \\
\text { Gear } 1\end{array}$ & $\begin{array}{l}\text { Coupled to } \\
\text { Gear } 2\end{array}$ & Linear actuator & $\begin{array}{l}\text { Connection } \\
\text { arm-body }\end{array}$ & $\begin{array}{l}\text { Coupled to Gear } \\
3\end{array}$ \\
$\begin{array}{l}\text { EXPECTED LOAD } \\
\text { KN }\end{array}$ & 0,5 & 1,0 & 1,0 & 1,0 & 2,0 \\
$\begin{array}{l}\text { MAX STATIC C } \\
\text { KN }\end{array}$ & 3,1 & 9,5 & 0,761 & 3,1 & 18,6 \\
$\begin{array}{l}\text { MAX DINAMIC C } \\
\text { KN } \\
\text { NOTES }\end{array}$ & 3,0 & 4,5 & 0,72 & 3,0 & 19,0 \\
\hline
\end{tabular}

The bearing embedded in the linear actuator does not match with the required performances, but the values are close enough to suppose that a better design and more optimized choice of bearing can easily solve the problem. The bearings coupled with Gear 3 are clearly oversized. In fact, they are supposed to withstand not only the direct drag forces, but also the moment generated by the aerodynamic forces on the arms.

\subsubsection{Gears}

To complete a first order analysis of the screws, we utilized a revisited version of the Lewis formula. The Lewis formula is a conservative calculation proposed for plain gear. It states that:

Where:

$$
\sigma=\frac{1}{m Y} \frac{F_{t}}{b}
$$

- $\mathrm{F}_{\mathrm{t}}$ is the tangent force calculated as torque on the minimum radius of the complementary gear.

- $\mathrm{Y}$ is the Lewis factor

- $\mathrm{b}$ is the gear thickness, that we consider equal to the minimum radius of the worm gear.

\footnotetext{
${ }^{15}$ http://www.skf.com
} 
The efficiency of the gear transmission is set by default at 0.5. We imposed that the teeth inclination of the complementary gear should be in a range between 5 and 15 degree. From such boundary we derived, for each gear a range of teeth number. The maximum torque generated from a $10 \mathrm{~mm}$ and $5 \mathrm{~mm}$ diameter gearbox is respectively $0.15 \mathrm{Nm}$ and $0.03 \mathrm{Nm}^{16}$. Using those parameters and the formula above we then analyzed for each gear the admissible number of teeth and the maximum output torque obtainable. Ferrium C61, with a $\sigma_{\mathrm{s}}$ of $1551 \mathrm{MPa}^{17}$, with the space degeneration paradigm applied before, we reduce the value to $1085 \mathrm{MPa}(70 \%)$. We also define a generic indefinite fatigue limit at $750 \mathrm{MPa}$.

Green section represents a range where no problem occur, yellow represents a range where fatigue phenomena may occur, while red section represent the range where plastic deformations or failure may occur.

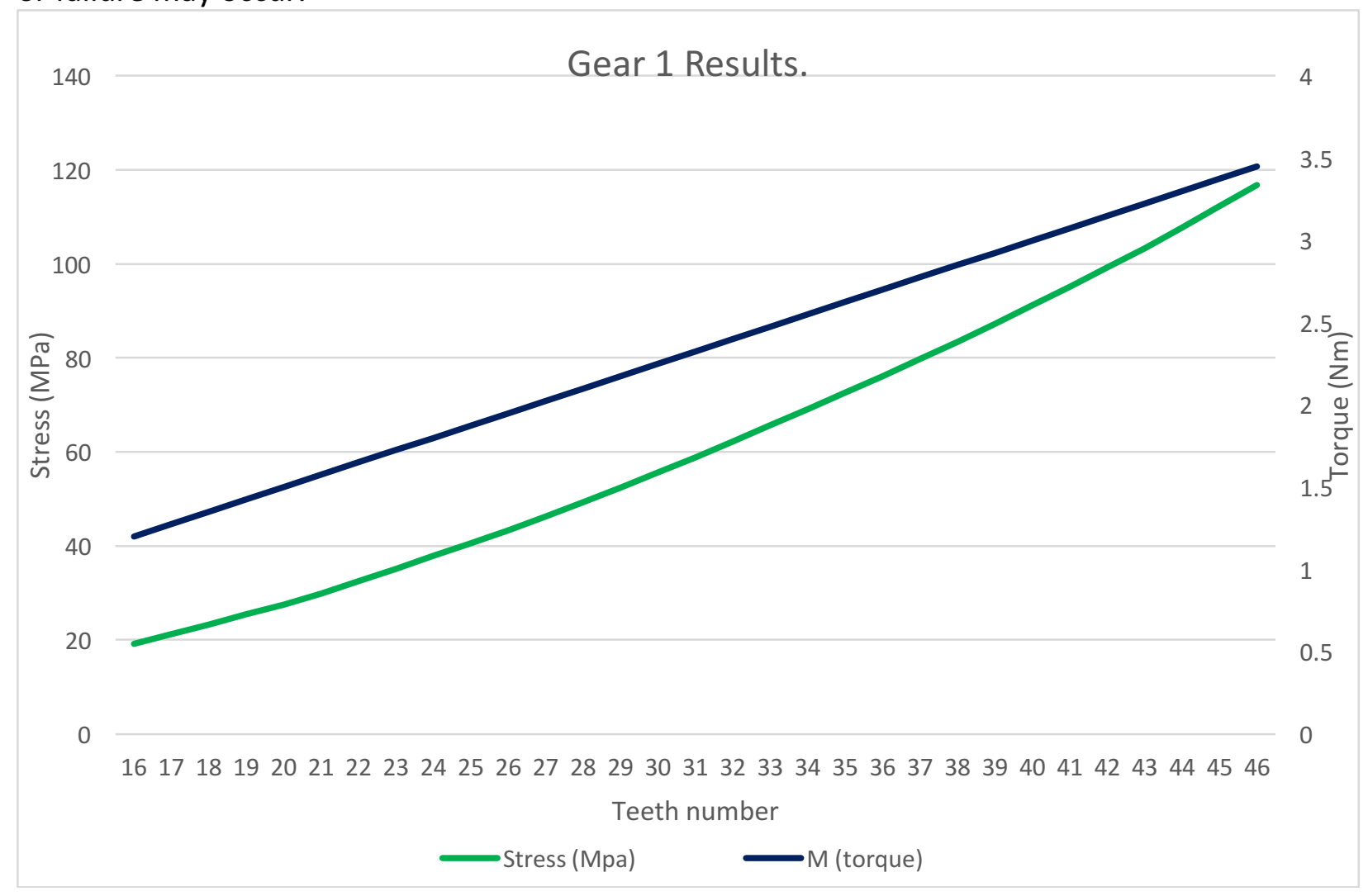

\footnotetext{
${ }^{16}$ http://www.maxonmotor.com

${ }^{17}$ http://www.questek.com/ferrium-c64.html
} 


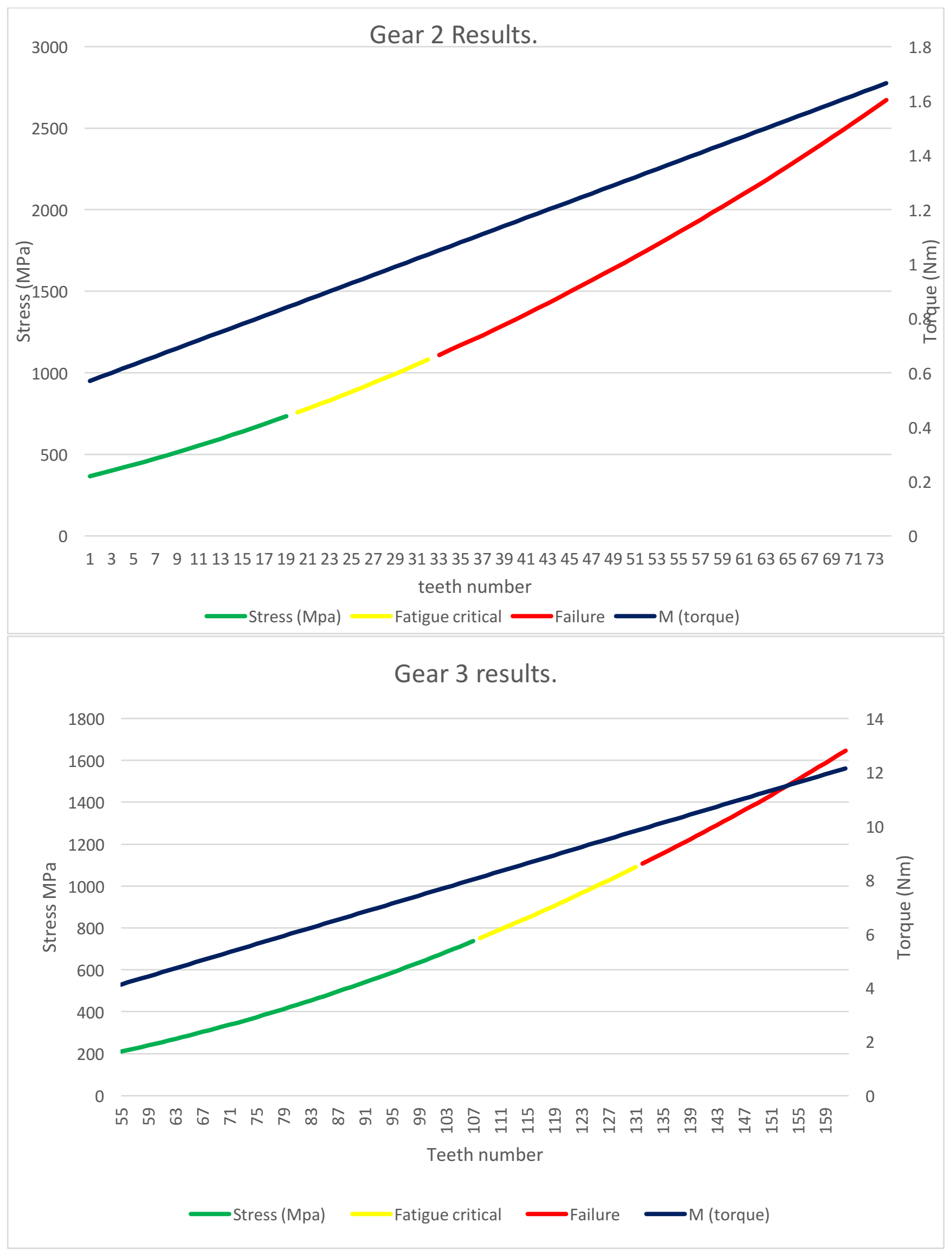




\subsection{D-printed Model}

While the first NIAC phase did not include the creation of an actuate prototype, to visualizes and clarify the DM kinematics, how the joints are actuated and where are located, we decided to produce a mockup, approximatively on scale, of the DM head. The mockup represents the front section only, and reproduces the sizes and the allowed degree of freedom. Instead of actuator the joints have been, when possible, spring loaded. Instead of a screw anchor a simple magnet has been inserted, to allow to see how a DM would look while climbing a surface.

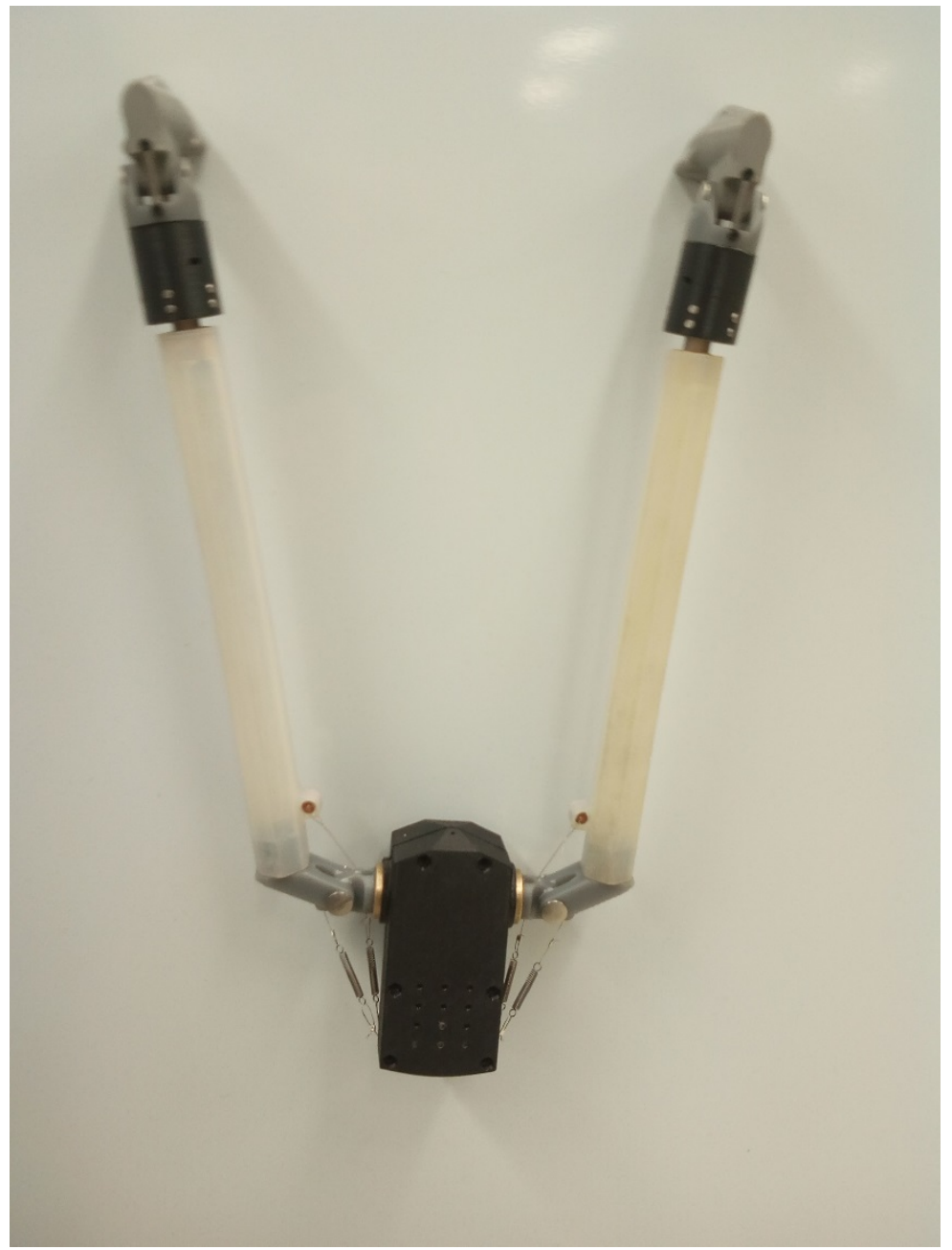

Figure 67 Mockup

\subsection{Ice screw end effector}

\subsubsection{Design}

Robotic ice climbing has not yet been accomplished, even on Earth. In cooperation with the LEMUR robot program, we have developed a robotic system for vertical ice mobility for potential future use on a DM platform. This Ice Screw End Effector (ISEE) is a prototype that 
seeks to demonstrate the mechanical feasibility of robotic ice climbing (Figure 1). It does not meet the all requirements identified for the DM (such as an overall diameter of less than $10 \mathrm{~cm}$, an aerodynamic shape, and environmental hardening) but provides a starting technology that can be miniaturized and improved.

The ISEE anchoring mechanism is based on alpinist ice climbing screws. Essentially, these are thread-cutting, self-driving screws with a hollow core. The hollow core allows ice to flow out the back of the screw, where it can be captured as a sample for analysis.

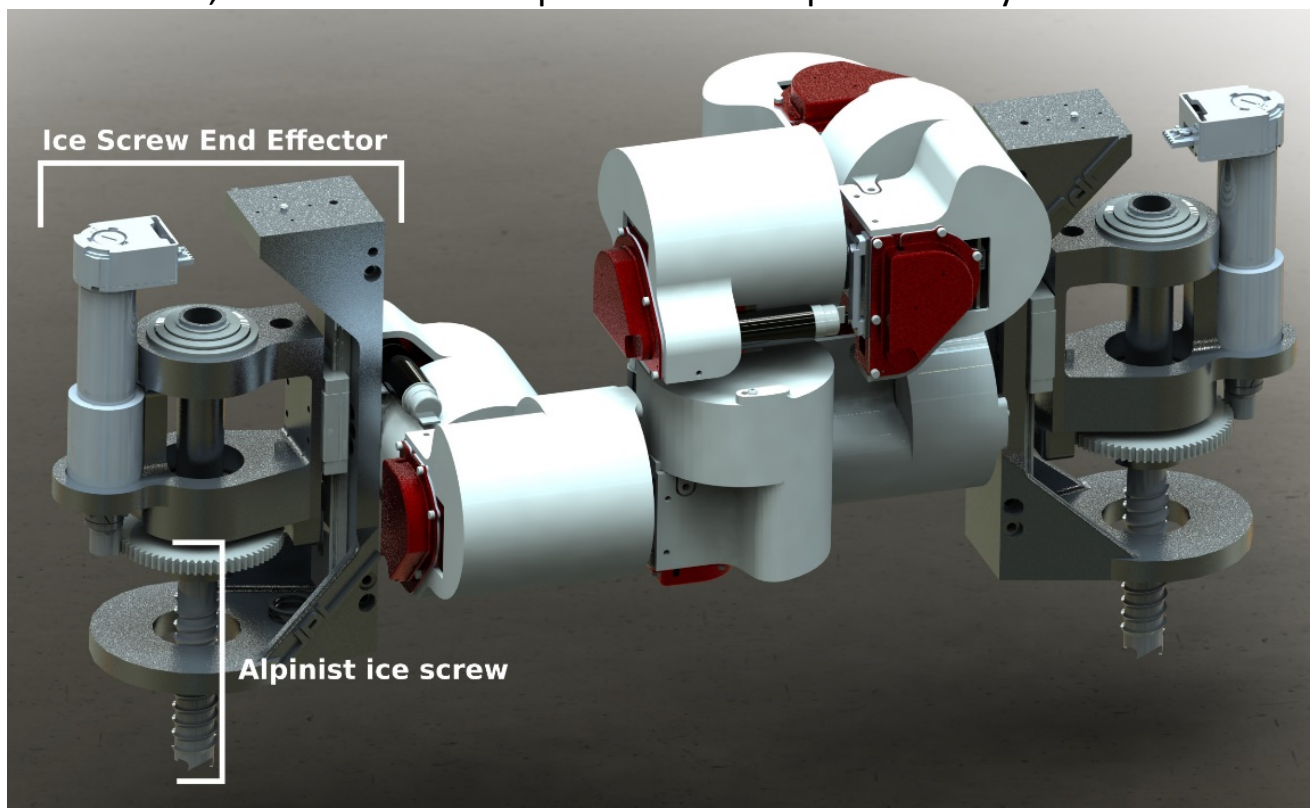

Figure 68. Ice Screw End Effectors (ISEE) shown in one possible robotic application. In this robot, two ISEE are used, one affixed to each end of a body composed of 7 rotary joints, modelled on an arm of JPL's LEMUR III robot. The robot would anchor one side at a time, "walking" up an ice wall.

\subsubsection{Assessment of screw insertion forces and torques}

Little data is available from previous work regarding required normal force into the ice to start an ice screw. Additionally, there is a lack of data regarding the torque necessary to rotate a screw as it cuts into the ice. We fabricated an ice screw end effector prototype and conducted a series of field and lab tests to determine these values.

Field tests were conducted in several fumarolic ice caves on Erebus volcano, Antarctica. The ISEE prototype was held against the wall by hand (Figure 69) and pushed to a given normal force. Normal force was estimated based on compression distance of a wave spring, which had graduated markings adjacent. For each experiment, screw insertion was re-attempted at increasing preloads $(30 \mathrm{~N}, 60 \mathrm{~N}, 120 \mathrm{~N}$ and $180 \mathrm{~N})$, until the screw insertion was successfully initiated. Each re-attempt was conducted in the same type of ice, $10 \mathrm{~cm}$ away from the previous insertion. 


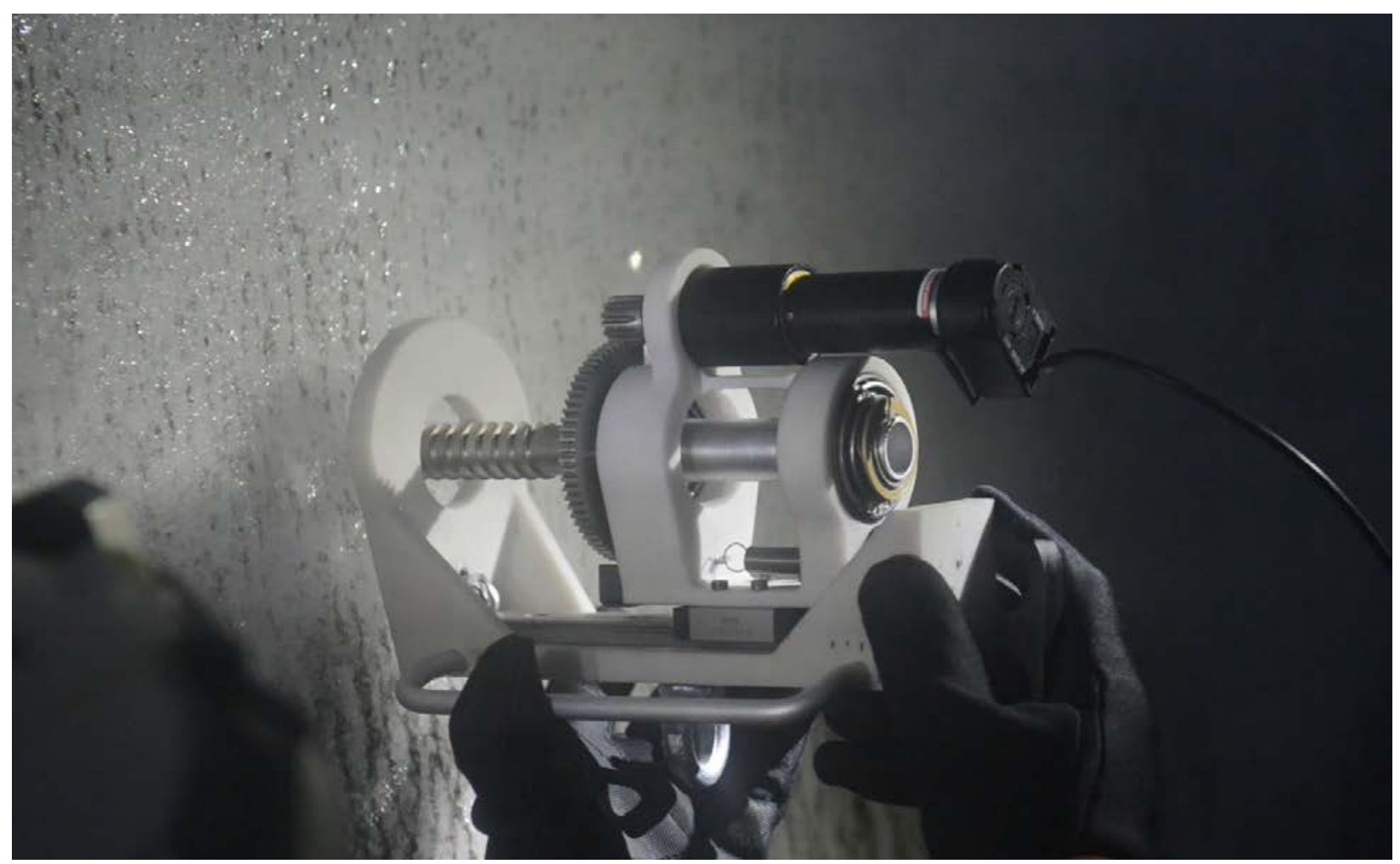

Figure 69. ISEE preload test in Helo Cave, a fumarolic ice cave on Erebus volcano in Antarctica.

All tests conducted in dense ice in fumarolic ice cave walls resulted in successful screw insertion. However, a test in hoarfrost crystals growing on a cave wall showed resulted in poor coupling with the wall. The screw could be easily inserted into and removed from the wall with no rotation. This highlights a potential concern of descent into vents on icy moons accumulation of hoarfrost may prevent effective anchoring. 


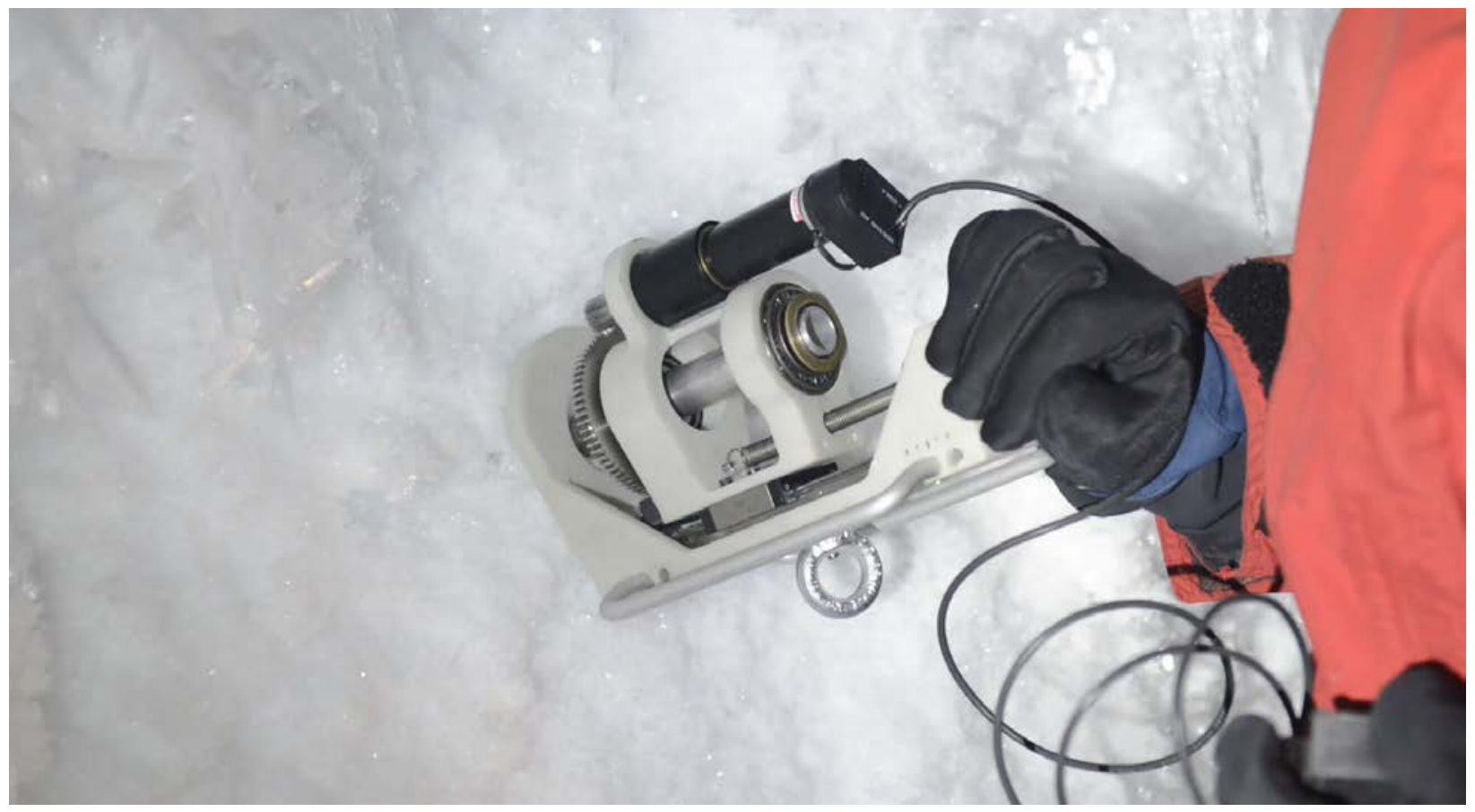

Figure 70. The ISEE did not anchor well in low density hoarfrost ice.

Similar tests were conducted on a testbed at JPL (Fig 5), where an ice block was secured to an ATI Gamma force torque sensor. Due to the small size of the ice blocks, these tests did not involve re-attempts; instead the selected preload value was maintained for 100 seconds and a fresh ice block was used for the next experiment. Three-axis force values and three-axis torque values were recorded at $10 \mathrm{~Hz}$ for all test stand experiments.

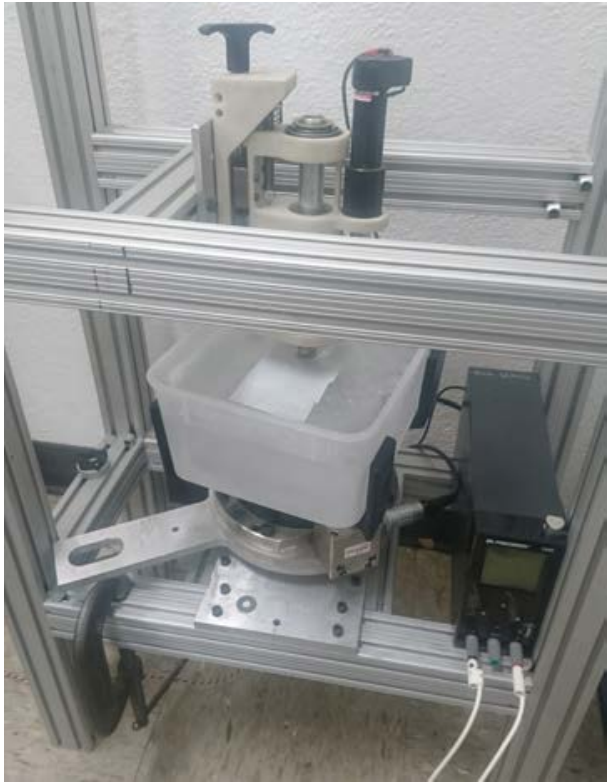

Figure 71. Ice screw end effector force / torque testbed, with ISEE mounted and ATI Gamma sensor beneath the ice block. The insulating sheet shown is placed beneath the screw before the experiment is initiated. 
The results (Fig 6) show that preloads above $30 \mathrm{~N}$ are generally required to initiate screw thread cutting. At low preloads, screw rotation causes stripping, destroying the threads. Earlier cold room tests had appeared to show screw insertion as low as $6 \mathrm{~N}$, but these low preload values may have been due to extra preload unaccounted for due to interaction of screw threads with the plexiglass lid of the experimental apparatus. More tests are recommended, especially testing low preload force with low rotation speed.

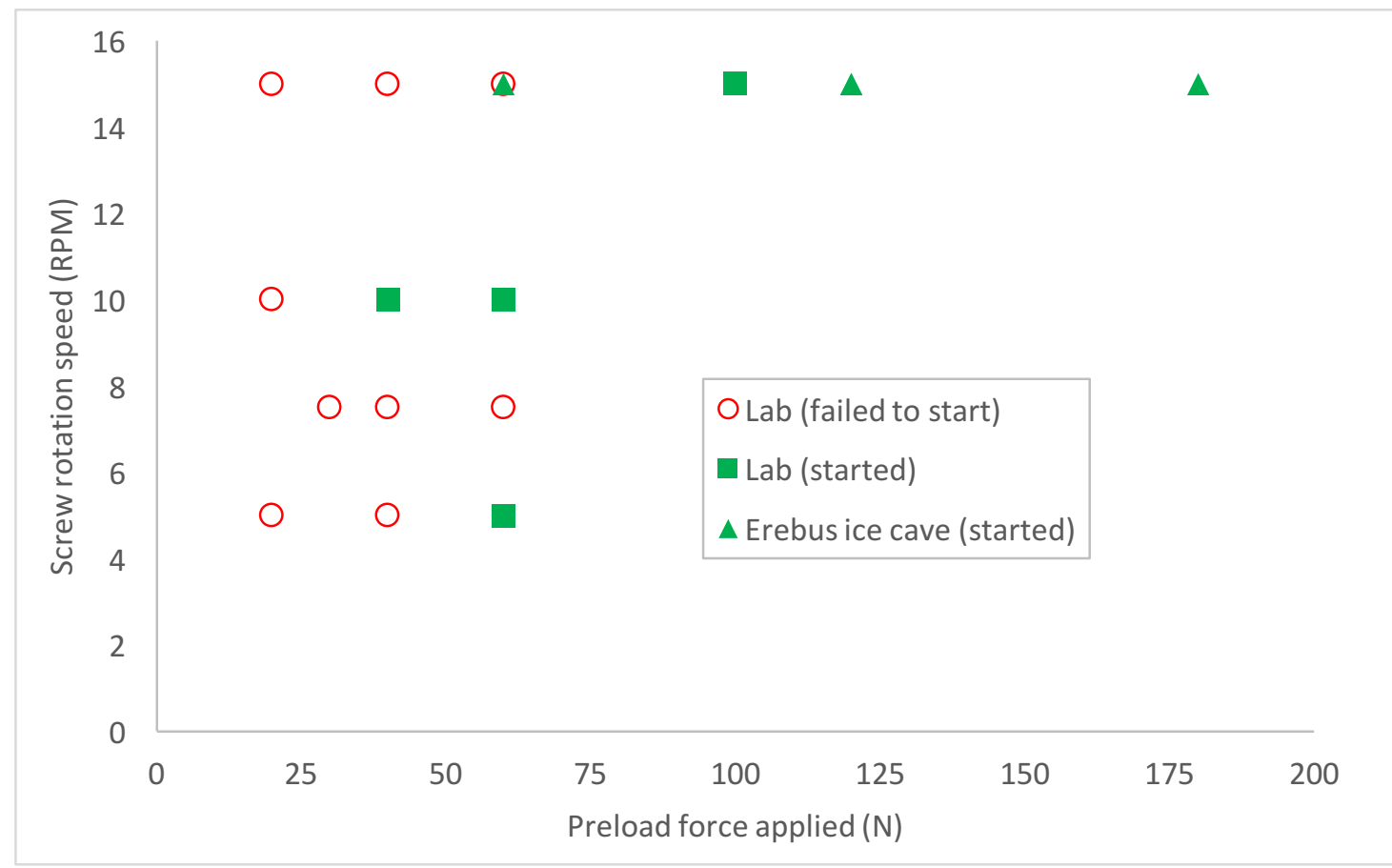

Figure 72. Results of screw thread initiation testing with normal preload force and speed of ice screw rotation

Torques imparted on the ice by the screw during experiments generally peaked between 1 and $4 \mathrm{Nm}$ (Figure 7). By observing the change in torque, it is possible to gain some insight into the process of thread stripping and the resulting failure of screw insertion. In Figure 7a, we see transmitted torque decrease after threads are destroyed at $1.25 \mathrm{Nm}$. 

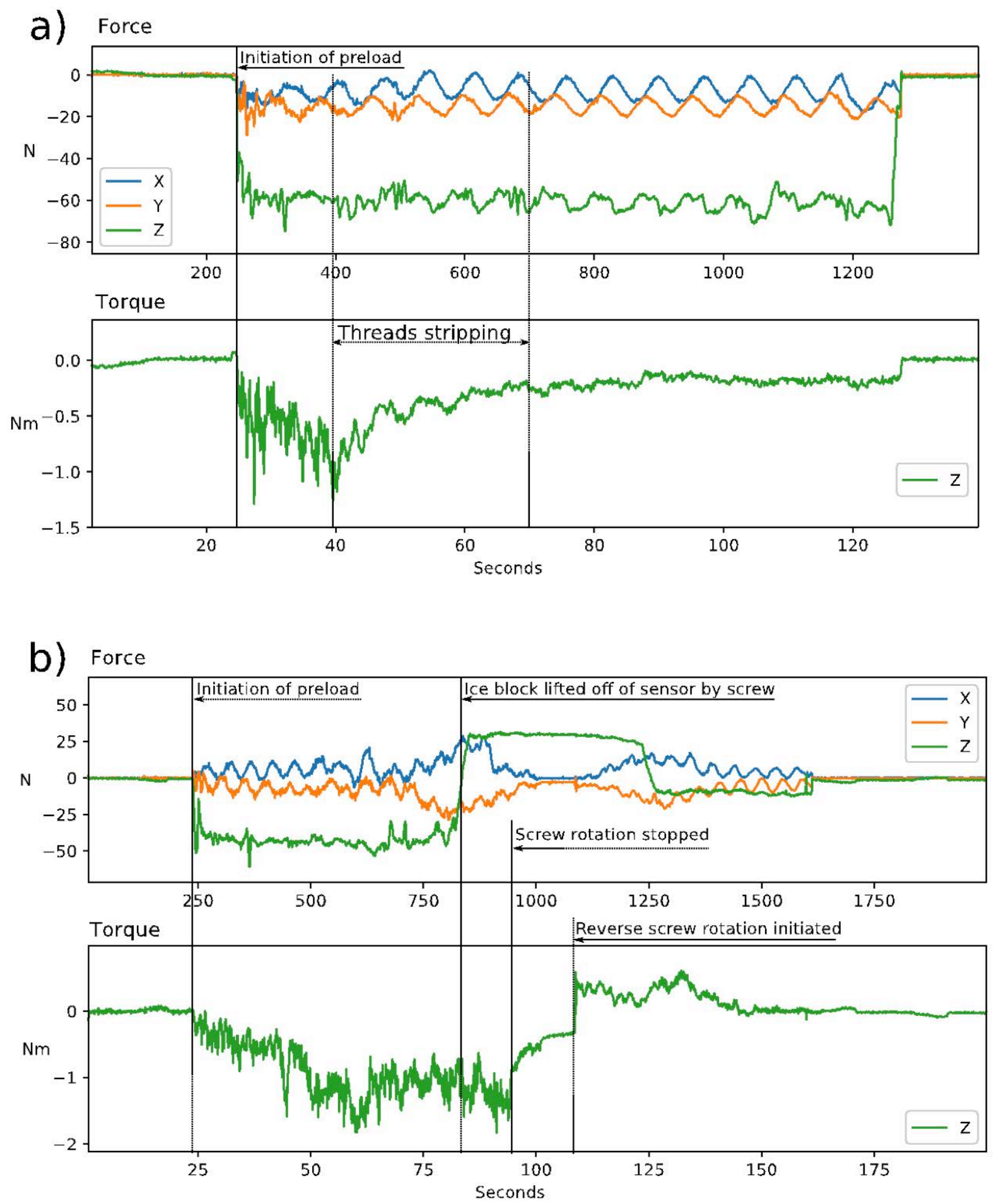

Figure 73. Data from the force / torque ice screw insertion test bed for two experiments, both conducted with a screw rotation rate of 10 RPM. a) Experiment with $60 \mathrm{~N}$ normal force, where screw insertion failed due to apparent thread stripping. b) Experiment at $40 \mathrm{~N}$ where screw was inserted successfully 


\section{Future Work}

The single greatest source of uncertainty in this concept is the eruption mechanism. In our preliminary analysis in the Phase I study, we found that EVE is likely feasible with the "boiling" models but the feasibility remains undetermined with the "cryovolcanic" models. Resolving between the two types of models is the highest priority, but it probably needs additional observations by a future orbiter such as Enceladus Life Finder (ELF).

However, refining the dynamic pressure and vent size estimates, as well as refining the DM design, could establish feasibility under cryovolcanic models, or at least get closer to it. As we discussed in Section 3.7, there are a number of additional analysis we can do with existing Cassini data, which will likely bring down the $10^{7} \mathrm{~Pa}$ upper bound. On the other hand, the refinement of the DM design, most importantly the optimization of aerodynamic shape, would bring up the current $10^{5} \mathrm{~Pa}$ limit. The drag coefficient of the DM design in Section 7 is about 1.5, while that of typical automobile is $0.2-0.3$. Whether or not the two bounds will meet is rest on future work.

There are several promising ideas of DM that have not been explored in Phase I. One is a "snake bot" design, which has additional middle sections for accommodating more instruments or a longer tether. Another idea is interchangeable middle section (i.e., swapping science instruments), which gives greater flexibility and robustness in operation.

Compared to mechanical capabilities, the required software capacities are less developed in general. For the reasons we discussed in Section 4.4, DM needs to be highly automated in order to complete the mission within a reasonable duration. Development of the key autonomy capabilities listed in the Autonomy Capability Roadmap (Figure 34) has a crucial importance.

Phase I mostly focused on DM, while using the proposed design of Europa Lander for SM and CRO. However, there are several differences between Europa and Enceladus which could significantly impact their capabilities, such as the available number of large moons for flyby in the planetary system, strength of radiation, and gravity. Performing detailed trade study for SM and CRO would be an important future work.

Interface between SM and DMs has not been explored in the Phase I study. SM will need mechanisms to store and deploy DMs, as well as to retrieve samples from sample return DMs. Developing an operation strategy to share resources (power, communication bandwidth, time of ground operators) between SM and DMs is also a future work.

Finally, while EVE's primarily target is Enceladus, a similar concept could be applied to Europa if vents exist. Adapting EVE for Europa ${ }^{18}$ would be an interesting direction. Probably the harsh radiation environment, which in turn results in a shorter mission duration, would be a major

\footnotetext{
${ }^{18}$ Conveniently, Europa and Enceladus shares the initial letter. Hence the acronym "EVE" will be applicable to Europa as well.
} 
challenge. EVE could also find terrestrial applications, such as exploration of geysers, crevasses, and ice caves ${ }^{19}$. Demonstrating the concept in Earth analogues would be a stepping stone to the future Journey to the Icy Moons.

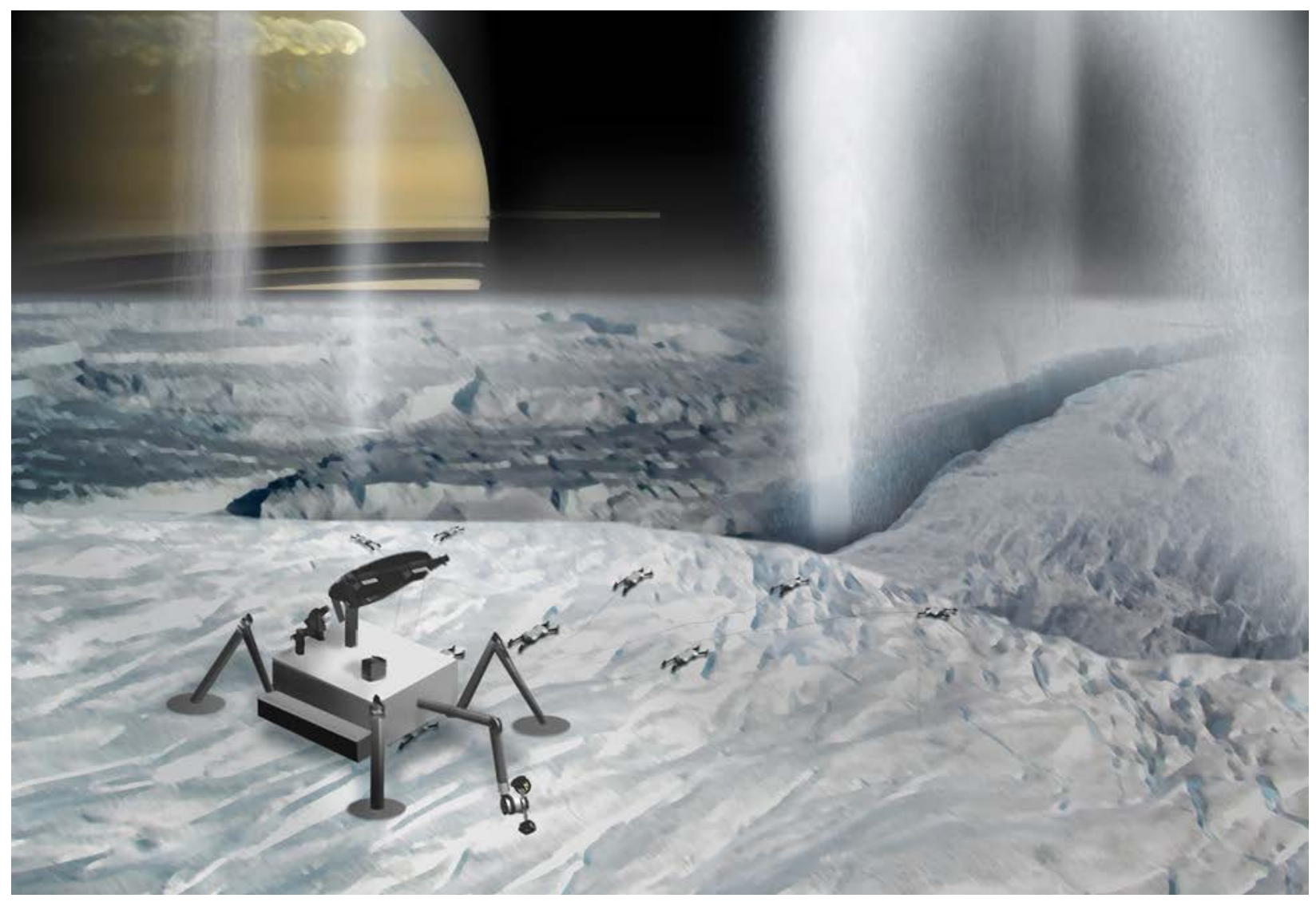

${ }^{19}$ Even more conveniently, Earth also has the same capital letter. 


\section{Bibliography}

Arakawa, M. \& Maeno, N., 1997. Mechanical strength of polycrystalline ice under uniaxial. Cold Regions Science and Technology, Volume 26.

Asbeck, A. T. et al., 2006. Climbing Walls with Microspines. s.I., s.n.

Barth, J. L., Dyer, C. S. \& Stassinopoulos, E. G., 2003. Space, Atmospheric, and Terrestrial

Radiation Environments. IEEE Transactions on Nuclear Science, 50(3).

Beverly, J. M., 2009. Ice Climbing Anchor Strength: An In-Depth Analysis, s.I.: s.n.

Beverly, M. J. \& Stephen , W., 2009. Ice Climbing Anchor Strength: An In-Depth Analysis.

[Online]

Available at: http://hmga.gr/storehouse/word-

acrobat/Ice\%20Climbing\%20Anchor\%20Strength\%20-\%20MRA\%202009\%20-\%20Marc\%20Beve

rly.pdf

Boston, P., 2016. Biovermiculation biopatterns as universal signatures of extant and extinct life.

s.l.:s.n.

Boston, P., 2016. Keynote talk, NIAC Symoposium. [Online]

Available at: https://livestream.com/viewnow/NIAC2016/videos/133893674

Boston, P. J., Spilde, M. N. \& Melim, L. A., 2001. Cave microbe-mineral suites: best model for

extraterrestrial biosignatures!. s.I., s.n.

Burge, S., 2009. The Systems Engineering Tool Box - Push Matrix (PM). [Online]

Available at: https://www.burgehugheswalsh.co.uk/uploaded/1/documents/pugh-matrix-

v1.1.pdf

Europa Lander Mission Concept Team, 2017. Europa Lander Study 2016 Report: Europa Lander

Mission, s.I.: s.n.

Fugueredo, P. et al., 2003. Locating Potential Biosignatures on Europa from Surface Geology Observations. Astrobiology, 3(4).

Goguen, J. D. et al., 2013. The temperature and width of an active fissure on Enceladus measured with Cassini VIMS during the 14 April 2012 South Pole flyover. Icarus, 226(1), pp. 1128-1137.

Hand, K. P. et al., 2017. Europa Lander Study 2016 Report: Europa Lander Mission, s.I.: s.n. Hansen, C. J. et al., 2011. The composition and structure of the Enceladus plume. Geophysical Research Letters.

Jones, D. S., Lyon, E. H. \& Macalady, J. L., 2008. Geomicrobiology of biovermiculations from the Frasassi cave system, Italy. Journal of Cave and Karst Studies, 70(2), pp. 78-93.

Kehl, F. et al., 2016. In-situ liquid extraction and analysis platform for Mars and ocean worlds. 3rd International Workshop on Instrumentation for Planetary Missions.

Kite, E. S. \& Rubin, A. M., 2016. Sustained eruptions on Enceladus explained by turbulent dissipation in tiger stripes. Geophysical Research Letters.

Liepmann, H. W. \& Roshko, A., 2002. Elements of Gasdynamics. s.I.:Dover Publications.

Lorenz, R. D., 2002. Thermodynamics of Geysers: Application to Titan. Icarus, 156(1), pp. 176183.

Matson, D. L., Castillo-Rogez, J. C., Davies, A. G. \& Johnson, T. V., 2012. Enceladus: A hypothesis for bringing both heat and chemicals to the surface. Icarus, 221(1), pp. 53-62.

McKay, C. P., 2004. What is life and how do we search for it in other worlds?. PLOS Biology. 
Mitchell, K., 2005. Coupled conduit flow and shape in explosive volcanic eruptions. Journal of Volcanology and Geothermal Research, Volume 136, pp. 223-240.

Parness, A., 2011. Anchoring Foot Mechanisms for Sampling and Mobility in Microgravity. s.l., S.n.

Parness, A., Frost, M., King, J. P. \& Thatte, N., 2012. Demonstrations of gravity-independent mobility and drilling on natural rock using microspines. s.I., s.n.

Porco, C. C. et al., 2006. Cassini Observes the Active South Pole of Enceladus. Science, 311(5766), pp. 1393-1401.

Porco, C., DiNino, D. \& Nimmo, F., 2014. HOW THE GEYSERS, TIDAL STRESSES, AND THERMAL EMISSION ACROSS THE SOUTH POLAR TERRAIN OF ENCELADUS ARE RELATED. Astronomical Journal, 148(3).

Sakurai, T. et al., 2015. Studies of melting ice using CO2 laser for ice drilling. elsevier, 8 October. Spitale, J. N. et al., 2015. Curtain eruptions from Enceladus' south-polar terrain. Nature, Volume 521, pp. 57-60.

Waite, J. H. et al., 2011. Enceladus' Plume Composition. s.I., s.n.

Warner, N., n.d. Personal communication. s.I.:s.n.

Wilcox, B. H., Carlton, J. A., Jenkins, J. M. \& Porter, F. A., 2017. A Deep Subsurface Ice Probe for Europa. Big Sky, IEEE, p. 2622.

Wilhelms, F., Kriews, M. \& Dick, D., n.d. The physical properties of ice with respect to laser light for environmental and industrial applications. s.I.:s.n.

Willis, P. A., Mora, M. F. \& Creamer, J. S., 2015. Implementation of microchip electrophoresis instrumentation for future spaceflightmissions. Analytical and Bioanalytical Chemistry.

Zimmerman, W., Bonitz, R. \& Feldman, J., 2001. Cryobot: An Ice Penetrating Robotic Vehicle for Mars and Europa. s.I., s.n. 
